Dissertation at the

Ludwig Maximilians Universität München

Faculty of Biology

\title{
Subcellular Mapping of Dendritic Activity in Optic Flow Processing Neurons
}

Submitted by

Elisabeth Hopp

May 27, 2015

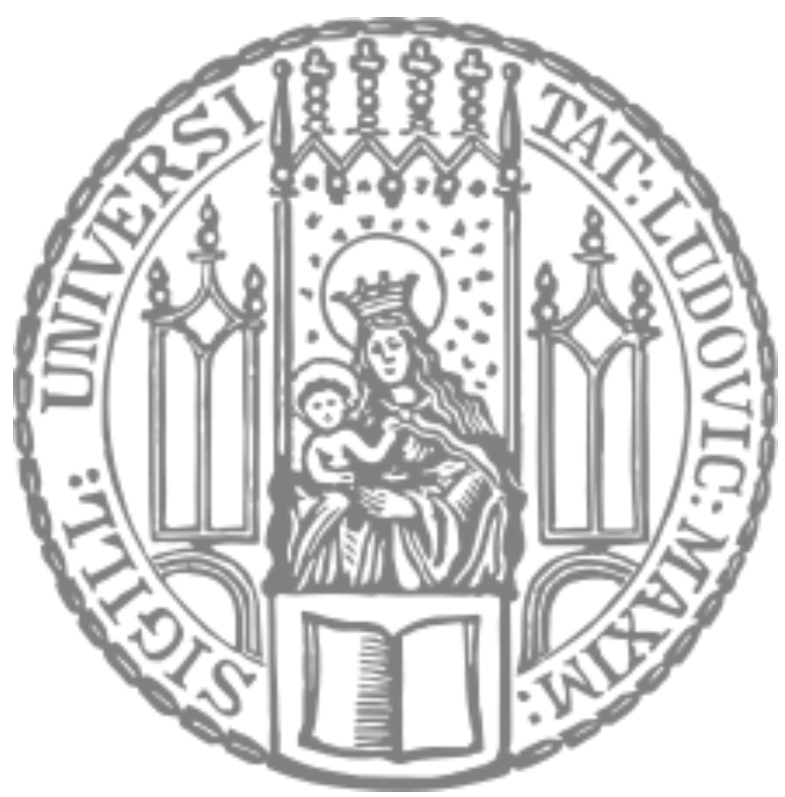



First reviewer

Prof. Dr. Alexander Borst

Second reviewer

Prof. Dr. Rainer Uhl

Oral defense

10/13/2015 

To Matt and Luke 



\section{Abstract}

Dendritic integration is a fundamental element of neuronal information processing. So far, few studies have provided a detailed picture of this process, describing the properties of local dendritic activity and its subcellular organization.

Here, I used 2-photon calcium imaging in optic flow processing neurons of the blowfly Calliphora vicina to determine the preferred location and direction of local motion cues for small branchlets throughout the entire dendrite. I found a pronounced retinotopic mapping on both the subcellular and the cell population level. In addition, dendritic branchlets residing in different layers of the neuropil were tuned to distinct directions of motion. Within one layer, local preferred directions varied according to the deflections of the ommatidial lattice. Summing the local receptive fields of all dendritic branchlets reproduced the characteristic properties of these neurons' axonal output receptive fields.

These results corroborate the notion that the dendritic morphology of vertical system cells allows them to selectively collect local motion inputs with particular directional preferences from a spatially organized input repertoire, thus forming filters that match global patterns of optic flow. These data illustrate a highly structured circuit organization as an efficient way to hard-wire a complex sensory task. 



\section{Zusammenfassung}

Dendritische Integration ist ein grundlegendes Element neuronaler Informationsverarbeitung. Bislang gibt es nur wenige Studien, die lokal dendritische Aktivität und ihre subzelluläre Organisation analysieren und somit ein detailliertes räumliches Bild dieses Prozesses vermitteln.

In der vorliegenden Arbeit analysiere ich mittels 2-photonen Calcium Imaging die lokale dendritische Aktivität von Neuronen der Fliege Calliphora vicina, die optischen Fluss verarbeiten. Ich bestimme die subzelluläre Verteilung von Vorzugsort und -richtung lokaler Bewegungsinformation für kleine Äste über den gesamten Dendritenbaum. Dabei ergibt sich eine ausgeprägte retinotope Abbildung sowohl auf subzellulärer als auch auf Zellpopulationsebene. Zudem weisen Dendritenäste in verschiendenen Schichten des umgebenden Neuropils unterschiedliche Vorzugsrichtungen auf, was sich bei zweischichtigen Zellen durch orthogonale Vorzugsrichtungen in den verschiedenen dendritischen Kompartimente äußert. Innerhalb einer Schicht variiert die lokale Vorzugsrichtung entsprechend der Krümmung des Ommatidienrasters über das Auge. Die Summe der lokalen dendritischen rezeptiven Felder spiegelt die charakteristischen Merkmale der rezeptiven Felder des axonalen Ausgangssignals dieser Zellen wider.

Diese Daten illustrieren, dass VS Zellen durch ihre spezifische dendritische Morphologie gerade diejenigen lokalen Bewegungsinputs aus einem räumlich strukturierten Inputrepertoire abgreifen, die zusammen einen bestimmten globalen Optischen Fluss Filter bilden. Damit geben VS Zellen beispielhaft wider, wie ein komplexer sensorischer Verarbeitungsprozess durch eine räumlich stark strukturierte Schaltkreisorganisation effizient genetisch angelegt sein kann. 



\section{Contents}

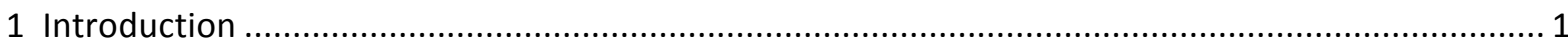

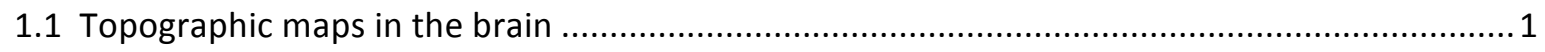

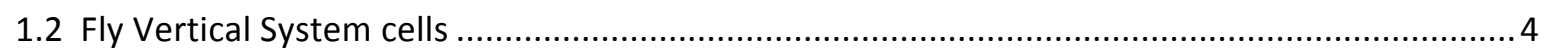

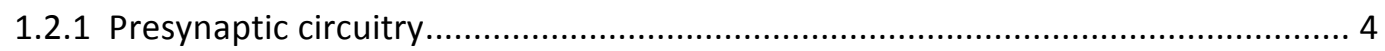

1.2.1.1 The Hassenstein-Reichardt detector ................................................ 4

1.2.1.2 Neuronal implementation............................................................. 7

1.2.2 VS cell response properties............................................................ 14

1.2.3 VS cell output and behavioral relevance.................................................. 15

1.2.4 VS cells in the lobula plate network ...................................................... 18

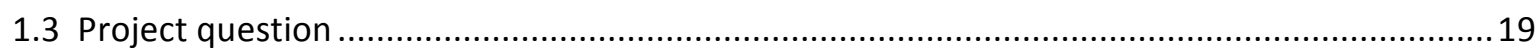

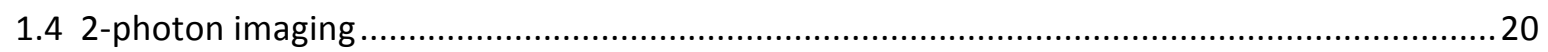

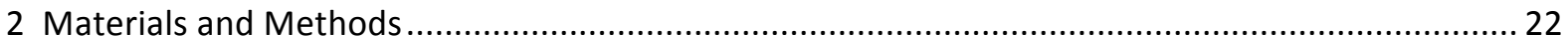

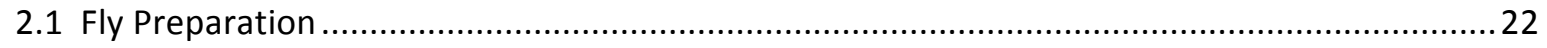

2.2 Stimulation setup and 2-Photon Microscopy.........................................................22

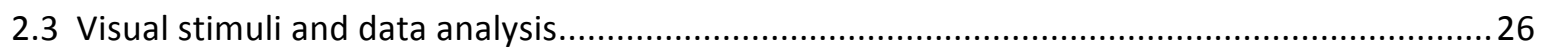

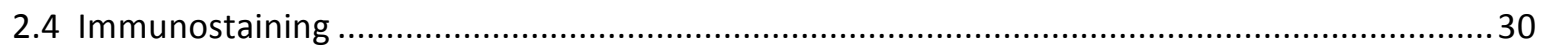

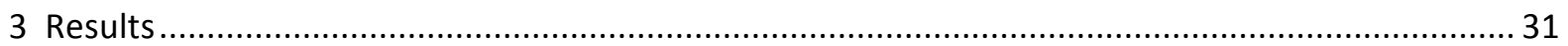

3.1 Mapping the receptive fields of small dendritic branchlets ............................................

3.2 Small dendritic branchlets have local receptive fields with clear direction selectivity ............35

3.3 Visual space is retinotopically mapped onto the lobula plate .........................................37

3.4 Bistratified cells exhibit a layered organization of local direction selectivity .......................40

3.5 The intralayer distribution of preferred directions follows the deflections of the ommatidial lattice

3.6 Local dendritic receptive fields constitute the building blocks of global optic flow filters ...... 45

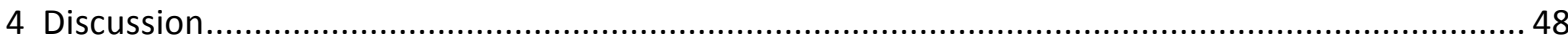




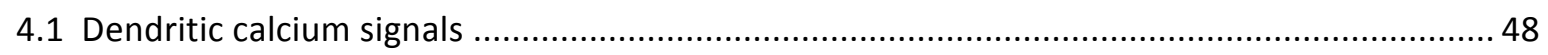

4.2 Deconvolution of the fluorescence traces ........................................................... 49

4.3 VS cell dendritic morphologies are adapted to collect appropriate receptive field components from a spatially organized input repertoire ...................................................... 50

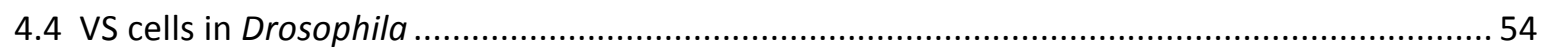

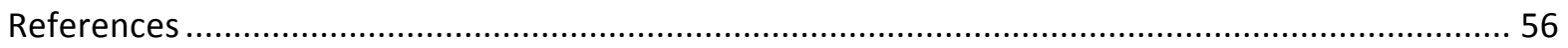

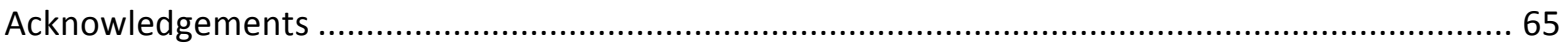




\section{Table of figures}

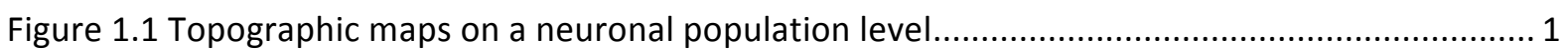

Figure 1.2 Lack of a subcellular input topography in mouse visual cortex .......................................... 2

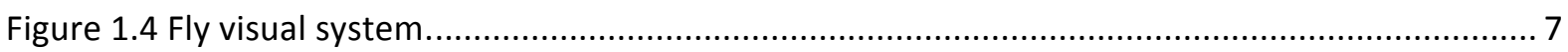

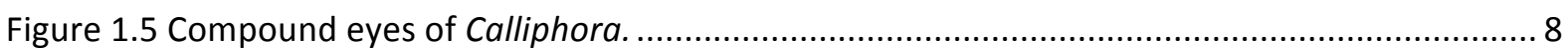

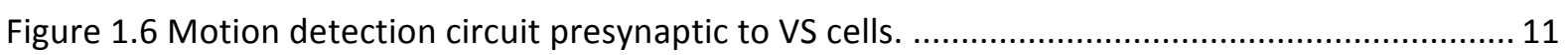

Figure 1.7 Electrophysiological response to motion in a VS cell........................................................ 14

Figure $1.8 \mathrm{VS}$ cell receptive fields resemble optic flows elicited by ego motion. ............................... 17

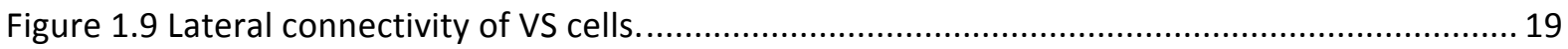

Figure 1.10 Laser excitation does not interfere with the visual system. ............................................ 21

Figure 2.1 Setup for 2-photon imaging under visual stimulation. .................................................... 23

Figure 2.2 Spectral separation of stimulus light and fluorescence signal. ........................................ 24

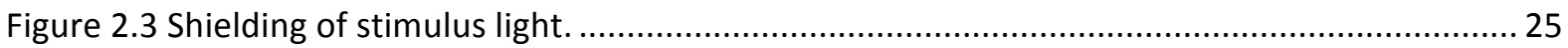

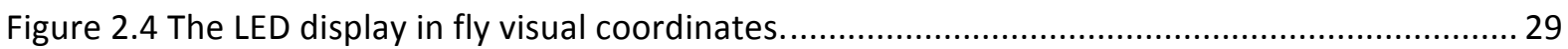

Figure 3.1 Mapping the receptive fields of small dendritic branchlets.............................................. 32

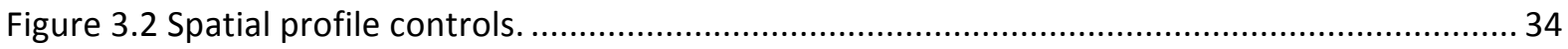

Figure 3.3 Small dendritic branchlets have local receptive fields with clear direction selectivity....... 36

Figure 3.4 Visual space is retinotopically mapped onto the lobula plate .......................................... 39

Figure 3.6 Bistratified cells exhibit a layered organization of local direction selectivity. ................... 42

Figure 3.7 The intralayer distribution of preferred directions follows the deflections of the ommatidial lattice.

Figure 3.8 Local dendritic receptive fields constitute the building blocks for global optic flow filters.

Figure 4.1 Retinotopy of the lobula plate. 



\section{List of abbreviations}

\begin{tabular}{|c|c|}
\hline CCD & charged coupled device \\
\hline $\mathrm{dCH}$ & dorsal centrifugal horizontal cell of the lobula plate \\
\hline DSI & direction selectivity index \\
\hline GUI & graphical user interface \\
\hline HS & horizontal system \\
\hline HSE & horizontal system equatorial cell of the lobula plate \\
\hline HSN & horizontal system north cell of the lobula plate \\
\hline HSS & horizontal system south cell of the lobula plate \\
\hline KAc & potassium acetate \\
\hline $\mathrm{KCl}$ & potassium chloride \\
\hline$K_{d}$ & dissociation constant \\
\hline L1-5 & lamina neurons $1-5$ \\
\hline LED & light emitting diode \\
\hline LP & lobula plate \\
\hline LPTC & lobula plate tangential cell \\
\hline $\mathrm{Mi}$ & medulla intrinsic neuron \\
\hline nAChR & nicotinic acetyl choline receptor \\
\hline ND & null direction \\
\hline OGB-1 & oregon green bapta 1 \\
\hline PBS & phosphate buffered saline \\
\hline PBT & phosphate buffered saline + triton \\
\hline PD & preferred direction \\
\hline PFA & paraformaldehyde \\
\hline РMT & photomultiplier tube \\
\hline RF & receptive field \\
\hline Rh1-6 & rhodopsin 1-6 \\
\hline ROI & region of interest \\
\hline sd & standard deviation \\
\hline T4/T5 & $\begin{array}{l}\text { bushy T-cells connecting the medulla and lobula, respectively, } \\
\text { to the lobula plate }\end{array}$ \\
\hline $\operatorname{Tm}$ & transmedullary neuron connecting the medulla to the lobula complex \\
\hline VGCC & voltage gated calcium channel \\
\hline vs & vertical system \\
\hline
\end{tabular}





\section{Introduction}

\subsection{Topographic maps in the brain}

Topographic maps are a prevalent, well-studied phenomenon in the organization of the brain. A famous example is the somatotopic map of the human primary sensory cortex, also referred to as 'homunculus'. This simplified illustration builds on the observation that nearby areas on the cortical surface respond to stimulation of neighboring body parts [1, Fig. 1.1a]. Apart from stimulus space, stimulus features have also been found to be orderly represented by the brain. In the visual cortex of shrews, cats and monkeys, for instance, the orientation of an object is systematically represented across the brain surface [2, Fig. 1.1b].

a

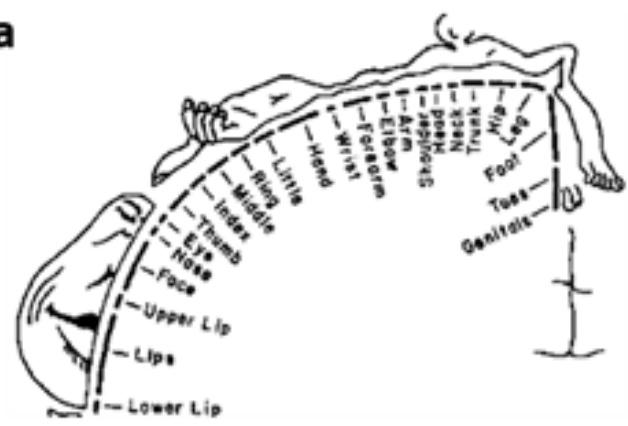

b

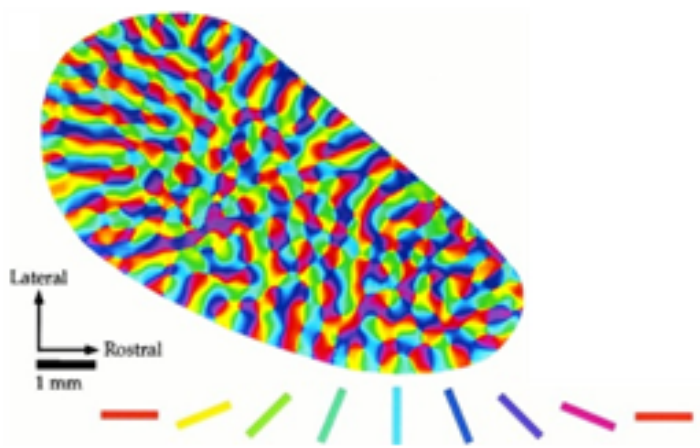

Figure 1.1 Topographic maps on a neuronal population level a Schematic drawing of the somatotopic map of the human sensory cortex [from 1] $\mathbf{b}$ Orientation preference map in area V1 of the tree shrew visual cortex. Orientation preference of each location is color-coded according to the key shown below [from 3]

However, such a topographic organization of sensory inputs has been mostly described on a neuronal population level. Few studies have investigated the subcellular distribution of sensory inputs: In the mammalian visual [4], vibrissal [5], as well as auditory [6] cortex, inputs tuned to different ranges of a specific stimulus parameter are scattered across the dendrite, lacking any particular spatial organization. In contrast, 
neurons in the mammalian retina [7], the vertebrate tectum [8], and the insect visual system [9] show evidence of a topographic input organization.
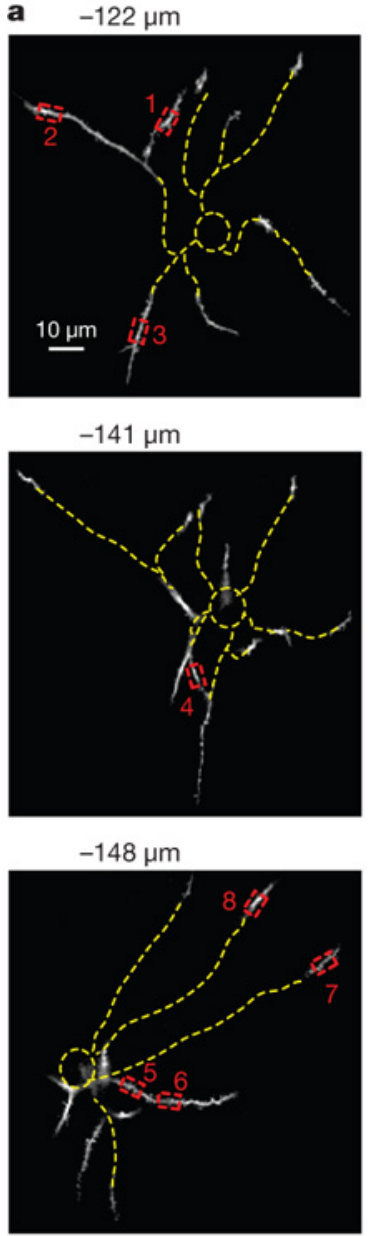

$-193 \mu \mathrm{m}$

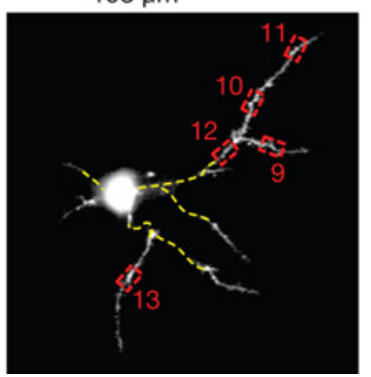

b

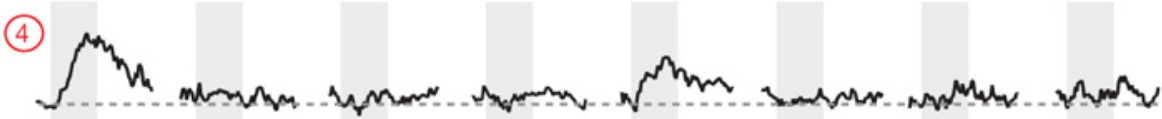

(12)

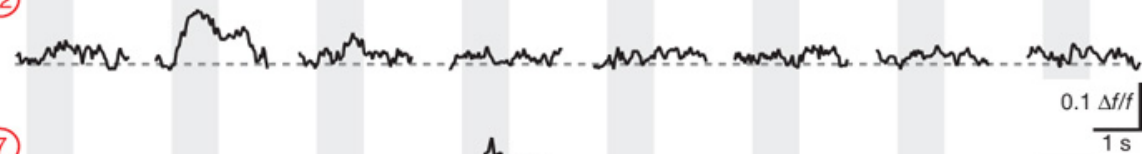
(7)

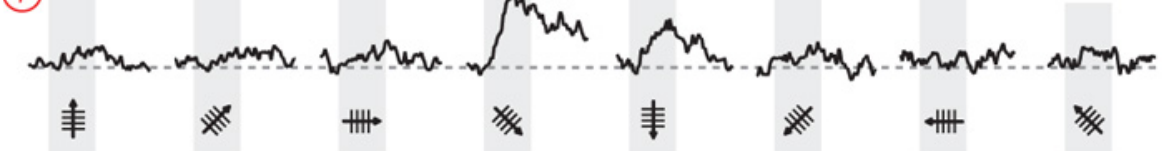

c
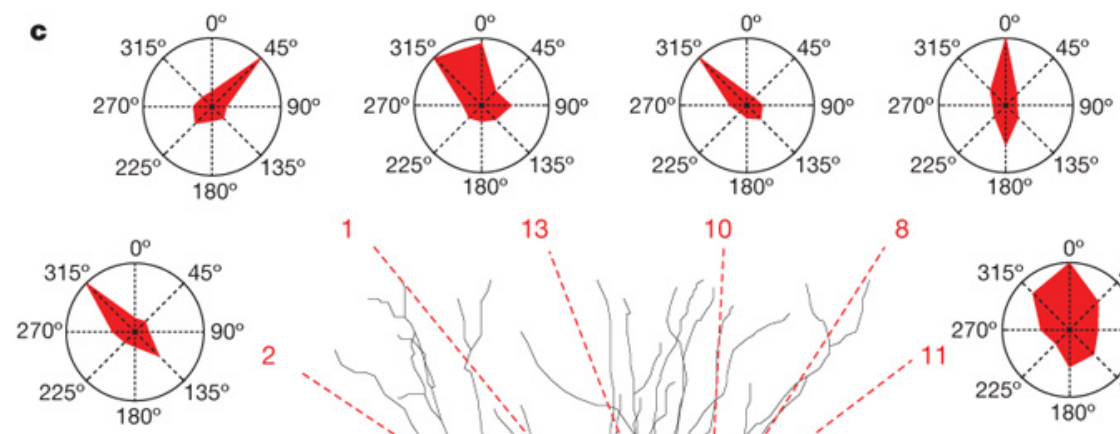

8
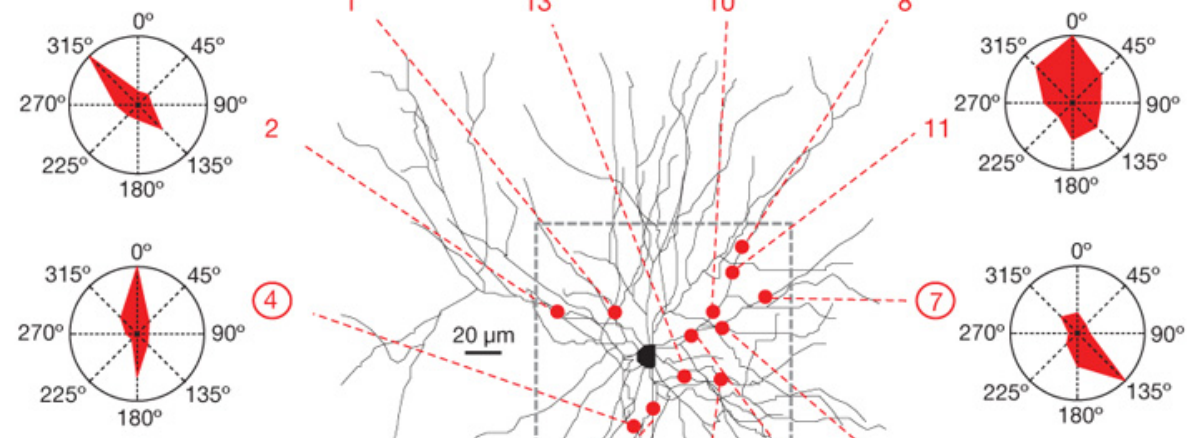

(4)
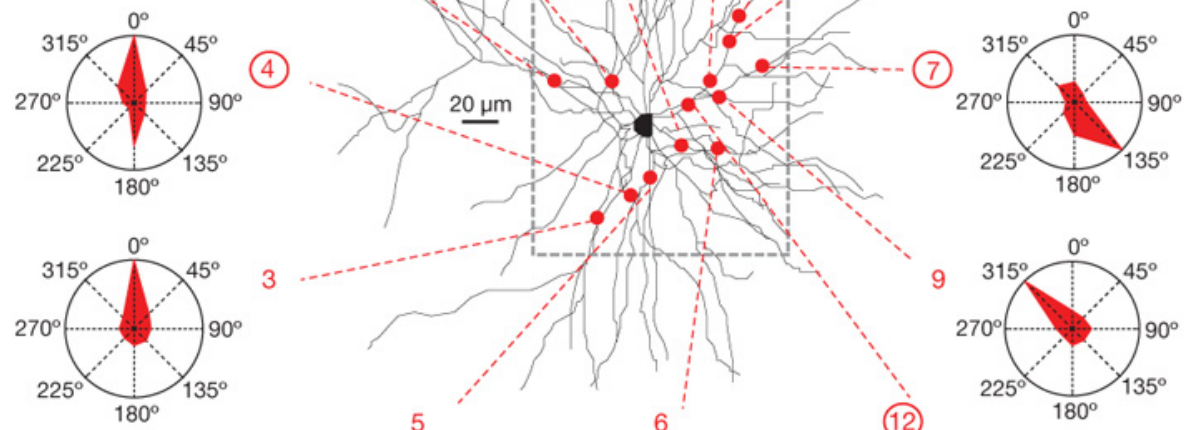<smiles>CC1CCC1</smiles>

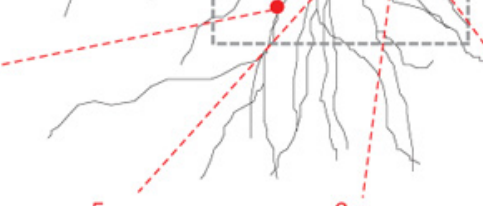

6

(12)
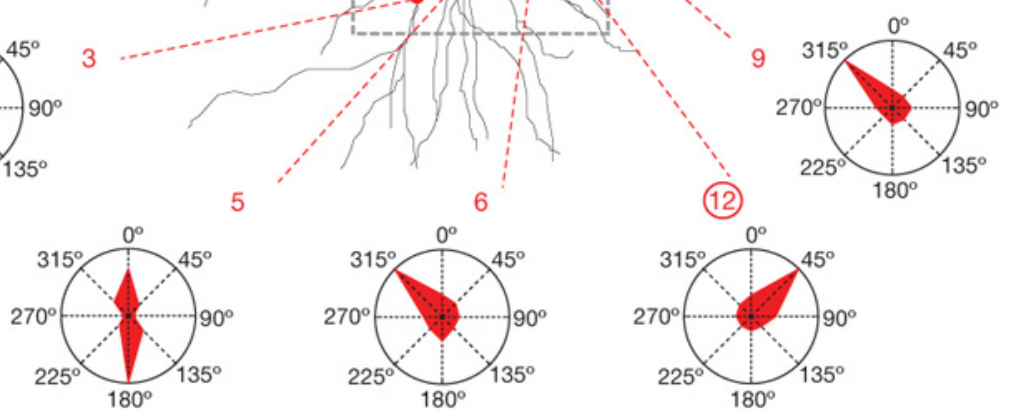

Figure 1.2 Lack of a subcellular input topography in mouse visual cortex a Four two-photon images of a neuron in layer $2 / 3$ of the mouse visual cortex, obtained at different depths under the cortical surface as indicated. Red dashed boxes indicate hotspots of local dendritic calcium signaling. $\mathbf{b}$ Local dendritic calcium signals evoked by drifting gratings of different orientations at three different dendritic sites as indicated in a. c Location of each hotspot indicated as a red dot on the Z-projection of the reconstructed dendritic tree. Red dashed lines point to the polar plot 
obtained for the corresponding local $\mathrm{Ca}^{2+}$ signals. The frame (grey dashed line) indicates the area of imaging. The output signal of the neuron was tuned for the vertical orientation [from 4].

The example of the disordered input arrangement on the dendrites of layer $2 / 3$ pyramidal cells in the mouse visual cortex in particular inspired this project. On these neurons, inputs with completely different tuning arrive right next to each other. At the same time, inputs with very similar tuning can arrive at opposite sides of the dendritic tree (Fig. 1.2). I was wondering what this would look like in the visual lobe of the fly. Given the crystalline structuring at different levels of the fly visual system - from the orderly arrangement of facets on the fly's compound eye (Fig. 1.5), which is mirrored in the columnar layout of its first visual neuropils, to the stereotypical shape and location of the direction selective tangential cells further downstream - I expected a very different picture. Furthermore, the study on visual cortex neurons does not consider all aspects of the visual input. It focuses on direction tuning, ignoring the spatial receptive fields of the inputs. By contrast, I aimed at comprehensively describing the subcellular distribution of a neuron's input, with all input features that collectively constitute its output.

A set of well-studied cells in the fly visual system was particularly suited for this endeavor: the vertical system (VS) cells. Three synapses downstream of the fly's photoreceptors, these cells have large, complex receptive fields, integrating local motion cues over a vast part of visual space. They have been studied intensely [for review, see 10], so their outputs were very well characterized, and a lot was already known about their synaptic inputs. In the following, I will give an overview of their presynaptic circuitry, their response properties, behavioral relevance and their embedding in the local network. 


\subsection{Fly Vertical System cells}

\subsubsection{Presynaptic circuitry}

\subsubsection{The Hassenstein-Reichardt detector}

For decades, only little was known about the presynaptic circuitry of VS cells. It was known that VS cells themselves exhibit direction selective responses to moving gratings, whereas direction selectivity is absent on the level of the fly's photoreceptors. The neural pathways that lie between long remained elusive. However, motion vision in flies has been extensively studied by the application and elaboration of a mathematical model called Hassenstein-Reichardt detector. I will start with a brief summary of this model, in order to provide a context for the description of what is currently known about the circuitry presynaptic to VS cells that implements local motion detection. The HassensteinReichardt detector was originally developed based on studying the turning tendency of a beetle, Chlorophanus, which was tethered to a holder and walked on a spherical Y-maze made from straws. A periodic, moving pattern surrounded the beetle, and at each bifurcation of the maze it could turn left or right [11]. Bernard Hassenstein's and Werner Reichardt's model for elementary motion detection describes the behavior of the beetle in a quantitative way and accounts for their observations in remarkable detail. In its simplest form, it consists of two mirror-symmetrical subunits. Each subunit (or halfdetector) processes luminance changes at two adjacent points in space. These values are multiplied, after one of them has been delayed by a low-pass filter. The outputs of the two half-detectors are finally subtracted [12] (Fig. 1.3). 


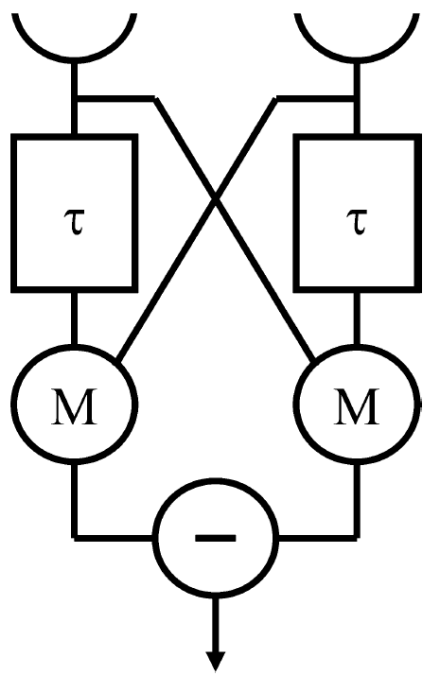

Figure 1.3 Reichardt model of elementary motion detection.

The model consists of two mirror-symmetrical subunits sharing the same two input lines. Within each subunit, the signal from one input is processed by a temporal low-pass filter with time constant $\tau$ and subsequently multiplied (M) with the instantaneous signal derived from the neighboring input. The signals from both subunits are subtracted (-), resulting in a directionally selective output [from 13].

A half detector generates a signal if the spatial arrangement of its delay and its direct line matches the direction of motion of an object passing by, that is, if the delayed signal coincides with the subsequently elicited direct signal at the multiplication stage. The signal is largest if the spacing between the two sampling points (the sampling base) relative to the time delay introduced by the low pass filter just compensates the velocity of the object. The detector as a whole will give a positive output for its preferred and a negative output for its non-preferred direction after subtraction of the output of the two half-detectors.

In principle, four different detector subtypes are conceivable. A luminance increment or 'ON'-signal at one point in space could be correlated with either a luminance increment or decrement ('OFF' signal) at a neighboring point. The four possible combinations are thus ON-ON, OFF-OFF, ON-OFF, OFF-ON. However, experiments using apparent motion stimuli (i.e., consecutive luminance increments or decrements at separate points in space that convey the illusion of a continuously moving object) indicated that only two of the four possible channels exist, one correlating luminance increments (ON-ON) and the other one correlating luminance decrements (OFF-OFF) [14, 15]. This makes sense from a biological standpoint since the movement of real objects will always lead to correlated ON or OFF signals at neighboring points in space. Still, one may wonder why there are two separate detectors, doubling wiring costs, and why not one single detector could implement the sign rule of multiplication, with the signal being positive when two positive as well as two negative brightness steps are correlated. 
However, it is hard to conceive how this could be implemented biophysically. Half-wave rectification of the input signal and splitting into an ON and OFF channel simplifies the problem considerably.

The Hassenstein-Reichardt detector model makes several predictions, which could be experimentally verified. Some of them even eluded its inventors, and have been studied much later [for review, see 16]. For example, fed with a moving sine grating, a Hassenstein-Reichardt motion detector produces an output that is not just linearly dependent on the pattern velocity, like a simple speedometer. Instead, its output increases as a function of image angular velocity, up to a maximum after which the response declines again. This maximum increases linearly as a function of the pattern wavelength. The ratio of pattern wavelength and velocity, i.e., the temporal frequency of a pattern that elicits the maximal response, therefore remains constant. This dependency of the detector on the properties of the pattern has been confirmed experimentally by electrophysiological recordings of large lobula plate neurons in both blowflies [17] and fruit flies $[18,19]$. Moreover, the Hassenstein-Reichardt model makes specific predictions regarding the transient response to grating motion, and it exhibits gain control, a property that was not noticed until fairly recently [for review, see 16]. The close fit between these predictions and the behavioral and electrophysiological observations make it very likely that a Hassenstein-Reichardt-type algorithm underlies motion detection in flies. While the range of plausible model parameters could be confined experimentally, its actual neuronal and biophysical implementation is still largely uncovered. 


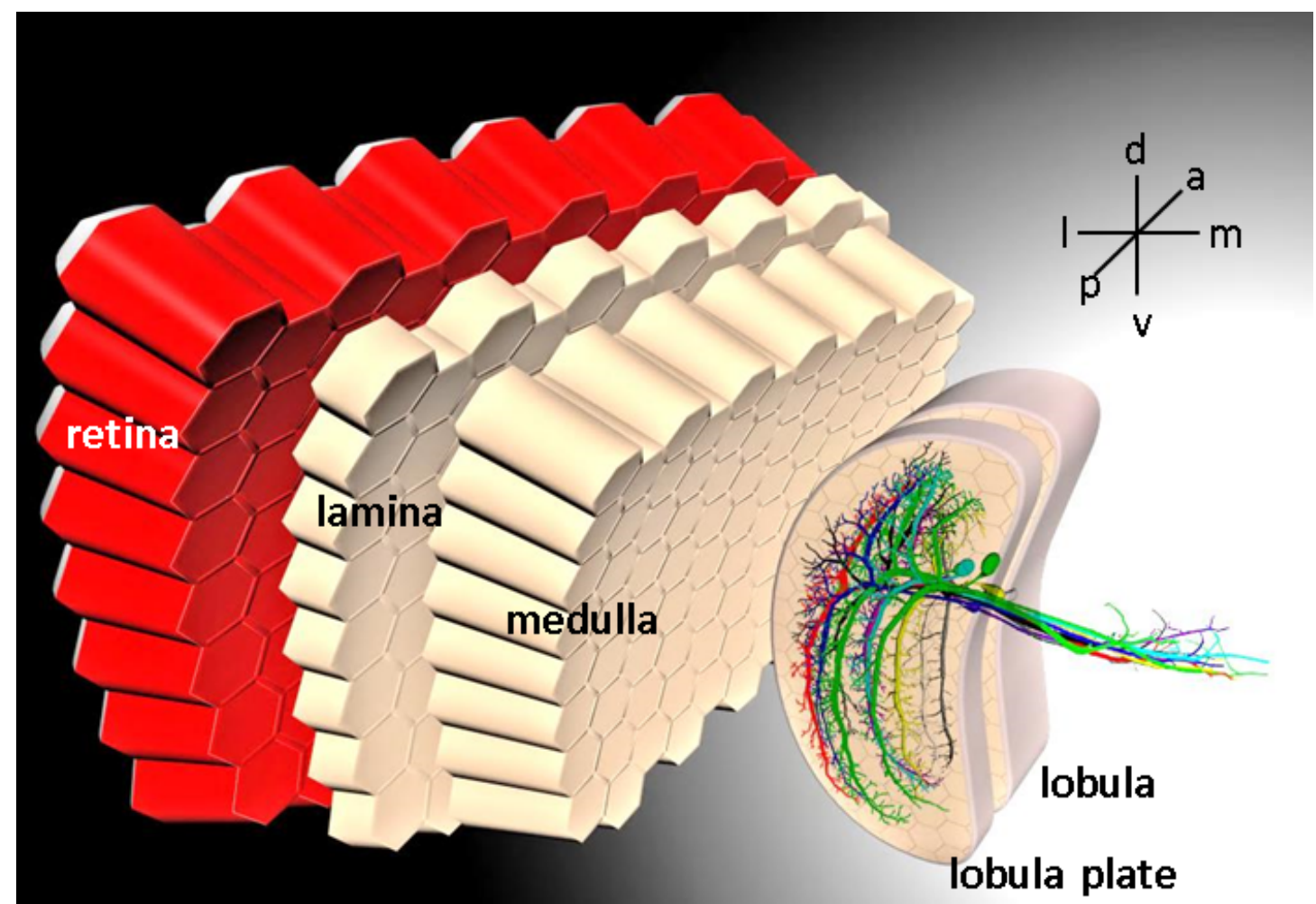

Figure 1.4 Fly visual system. The schematic shows the retina, lamina, medulla, lobula and the lobula plate with the VS cell population [modified from 20]. I, lateral, m, medial, d, dorsal, $v$, ventral, $p$, posterior, a, anterior.

\subsubsection{Neuronal implementation}

A major part of the fly's brain is devoted to visual processing. This part is called 'optic lobe' and can be anatomically subdivided into the lamina, medulla and lobula complex, composed of the lobula and the lobula plate (Fig. 1.4). All these neuropils have a columnar layout, matching the composition of the fly's compound eye into so-called ommatidia [21]. This layout is visible on the surface of the eye as an array of small, hexagonal faces called facets (Fig. 1.5). The retina consists of $\sim 5000$ ommatidia in Calliphora [22], with an interommatidial angle of 1-3 angular degrees, depending on the location on the eye [23]. Each ommatidium contains eight photoreceptor cells, R1-8, behind its own small lens. R7 and R8, the inner two receptor cells, are mainly involved in color vision [24, 25], whereas R1-6 all contain the same light-sensitive pigment, rhodopsin 1 (rh1), and feed into motion detection circuits [26-28]. Rh1 has two absorption peaks, 
one in the UV and one in the blue-green range [29, also compare Fig. 1.10]. R1-6 in the same ommatidium have different optical axes, depending on their location behind the facet lens. However, different receptor cells in neighboring ommatidia share an optical axis, following a regular, geometric pattern. The axons of those six neurons that share an optical axis converge on the same column in the lamina [30], a principle called "neural superposition". Consequently, one lamina column, also called cartridge, receives input from six different ommatidia, and the R1-6 in each ommatidium project to six different cartridges. This connection scheme preserves visual acuity while increasing sensitivity [31]. When activated by light, the photoreceptors depolarize [for review, see 32, 33] and release the neurotransmitter histamine at their synaptic terminals located in the lamina [34].

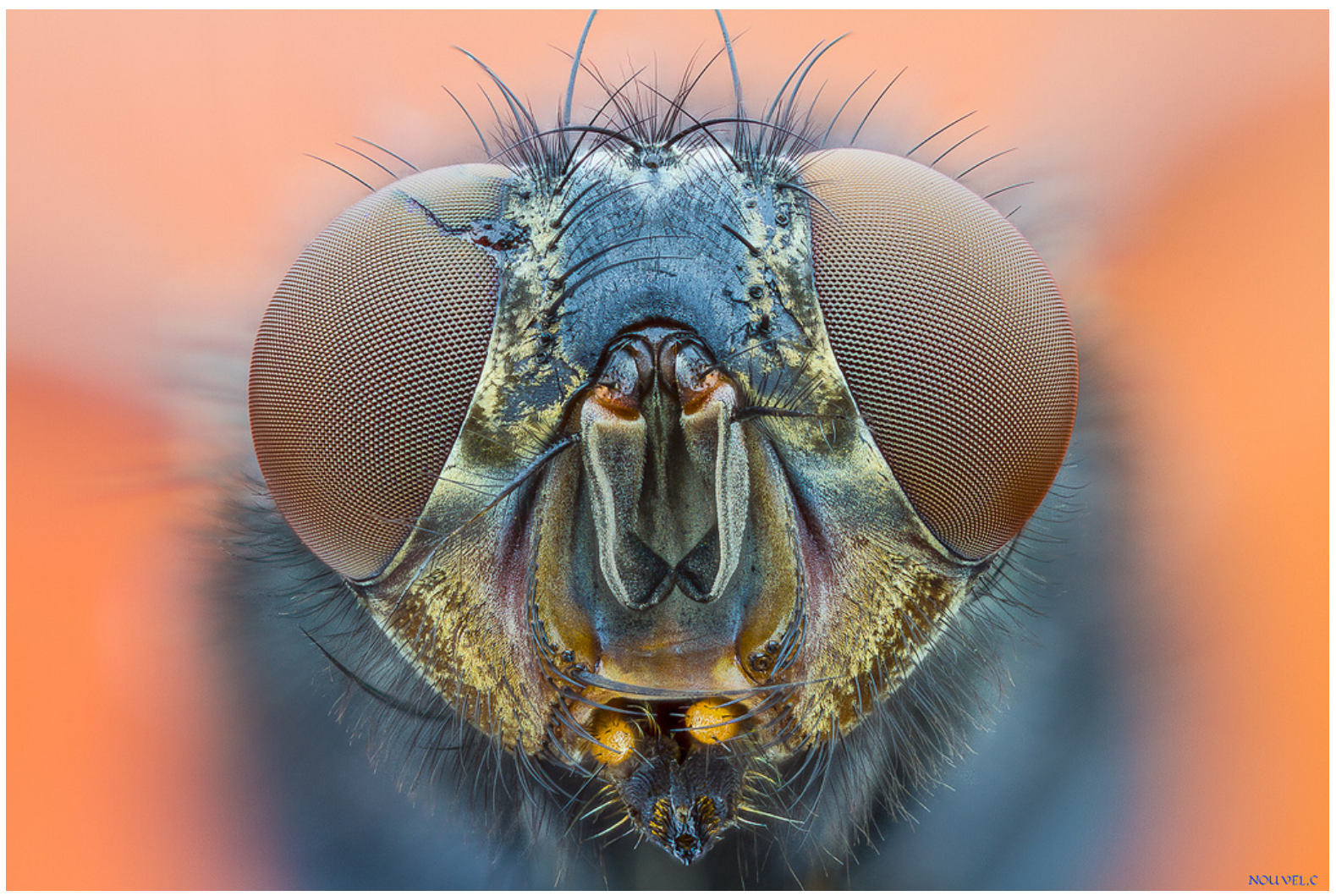

Figure 1.5 Compound eyes of Calliphora.

[modified from https://www.flickr.com/photos/77350506@N04/8971480924/in/photostream/]. 
In the lamina, the main targets of the photoreceptor cells are the large monopolar cells L1 and L2 and amacrine cells [35, 36]. The two largest monopolar cells, L1 and L2, are necessary and largely sufficient for motion detection. This has first been concluded from a behavioral study that measured optomotor responses while blocking synaptic transmission in L1 and L2, or while restoring synaptic transmission only in L1 and L2 in a background of impaired histamine receptors on cells postsynaptic to the photoreceptors [37]. Blocking either L1 or L2 separately and testing the response to motion stimuli electrophysiologically in downstream cells revealed a functional segregation between the two cell types: blocking only L1 eliminates the electrophysiological responses to a bright moving edge on a dark background in downstream neurons, but leaves the response to a dark edge on a bright background unaffected. Blocking L2, on the other hand, eliminates the response to dark moving edges [38]. A behavioral study later confirmed these results [39]. This suggests that at the level of the lamina, the signal from the photoreceptor cells is split into separate ON and OFF channels, transmitted by L1 and L2 cells, respectively. More precisely, combined apparent motion and genetic silencing experiments showed that the L1 channel exclusively deals with ON-ON signals, and the L2 channel exclusively processes OFF-OFF signals [40].

Histamine release by the photoreceptor terminals in the lamina opens histamine gated chloride channels on the large monopolar cells [41]. As a consequence, the signal is sign-inverted, and L1 and L2 hyperpolarize in response to light. This hyperpolarization consists of a strong, transient component, followed by a sustained component, and a rebound excitation when the light is switched off [42]. L1 and L2 both adapt over a broad range of luminances, maintaining an almost unchanged contrast sensitivity [43, 44]. Single cell transcript profiling indicates that they transmit their signals via different transmitter types to the medulla, L1 being glutamatergic and L2 cholinergic [45]. The OFF pathway also involves another cholinergic lamina monopolar cell, L4 [45], that seems to be crucial for motion processing [46]. L4 is reciprocally connected with L2 and connects adjacent columns in the lamina and medulla [35]. Moreover, the lamina monopolar cell L3 appears to play a role in OFF motion detection $[47,48]$. 
The next neuropil downstream, the medulla, again exhibits a columnar structure, matching the lamina in the number of columns [21]. Perpendicular to this columnar layout, the medulla also has a layered structure, with ten strata, M1-M10. All lamina neurons, except the lamina intrinsic amacrine cells, send their axons to the medulla and ramify in its different layers $[45,49]$. In addition, each medulla column houses at least 60 different cell types. Based on their anatomy, these cells can roughly be categorized into medulla intrinsic (Mi) neurons, which are confined to the medulla, transmedullary ( $\mathrm{Tm})$ cells, which connect the medulla to the lobula, the Y-shaped TmY cells, which connect the medulla to both the lobula and the lobula complex, and T4 cells, which connect layer M10 of the medulla to the lobula plate $[21,50]$. In addition, lobula T5 cells connect the lobula to the lobula plate. Based on anatomical costratificaton patterns, two functionally specialized pathways for motion detection were proposed early on: one pathway containing L1, Mi1, and T4, and a second one containing L2, Tm1, and T5 [51]. 
a

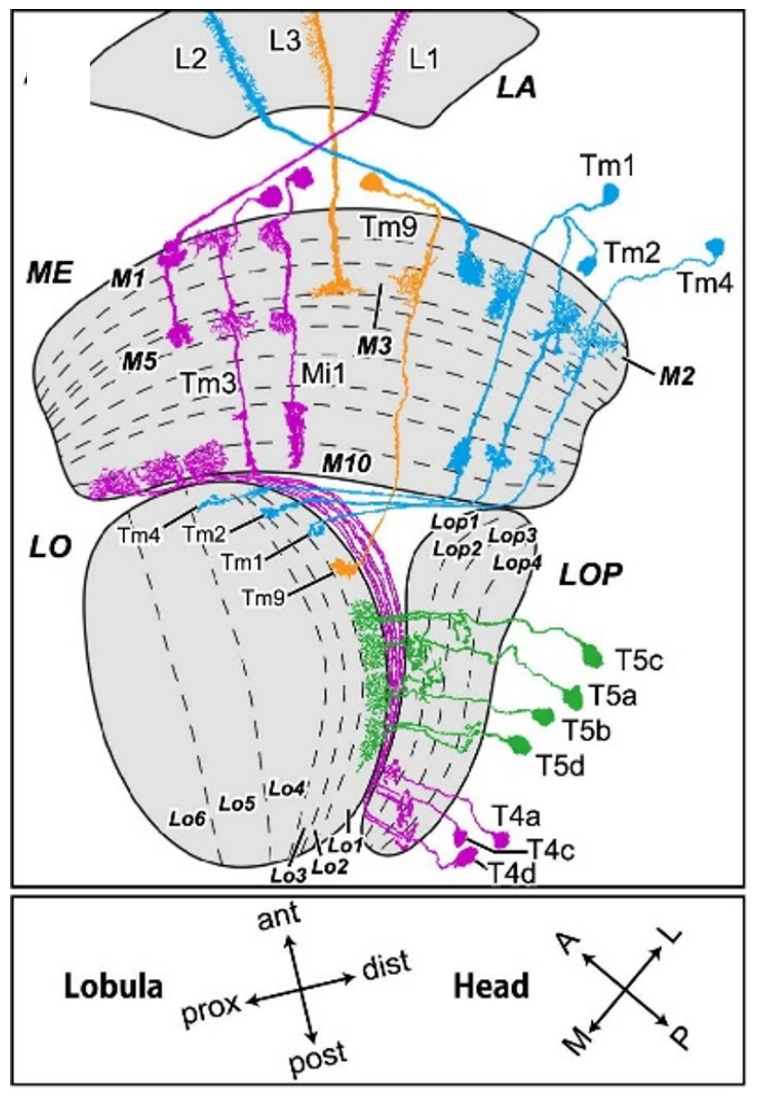

b

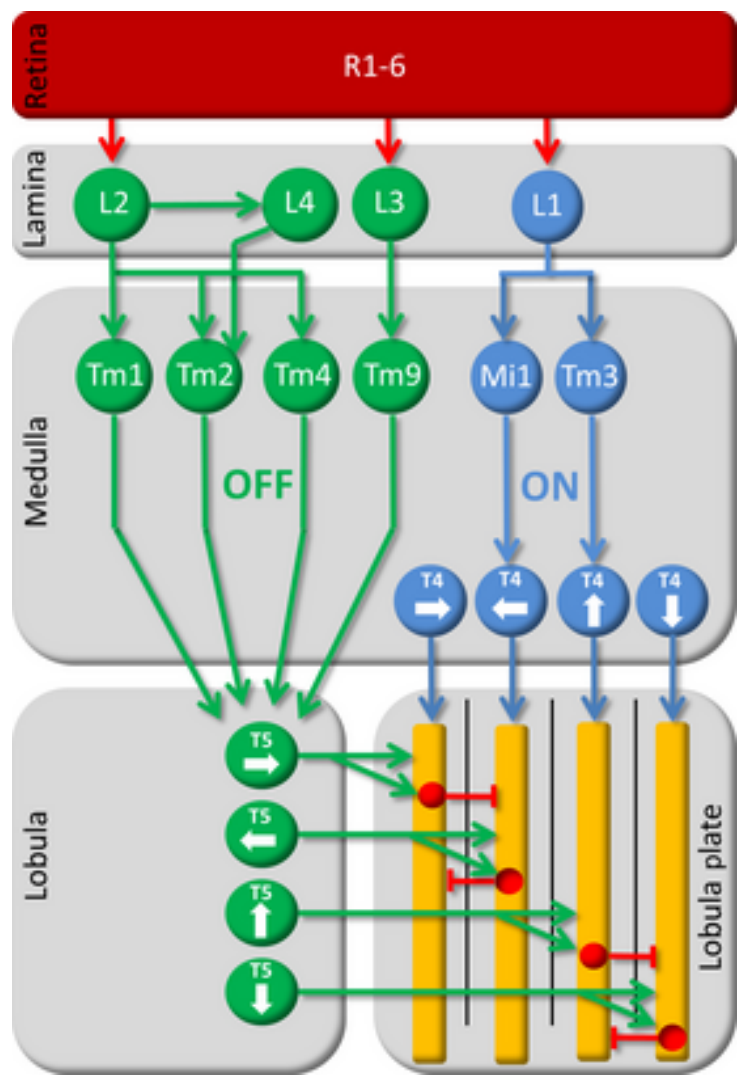

Figure 1.6 Motion detection circuit presynaptic to VS cells. a Drawings of the different cell types involved in motion detection. T4 receives input from the L1 pathway (magenta). T5 receives input from the L2 (cyan) and the L3 (orange) pathway. T4 and T5 both project to four layers in the lobula plate (LOP), Lop1-4, via lamina (LA) and medulla (ME). Inputs to T4 in the proximal medulla are shown only in part for clarity, with the terminals of Tm3 in the lobula (LO) omitted. In the lobula plate, T4 and T5 synapse onto VS and other tangential cells (not shown). Orientation markers are as follows: the lobula is rotated $90^{\circ}$ with respect to the medulla so that its anterior (ant) direction points toward the medulla and its posterior (post) toward the head's midline (M). The lobula's Tm cell inputs enter at its distal strata (dist), roughly toward the eye's lateral edge (L), and extend proximally (prox). The lobula's anterior edge receives input from frontally directed ommatidia and lamina cartridges, toward the head's anterior (A), after two inversions of the pathways through the chiasmata between lamina and medulla, and between medulla and lobula [from 52]. b Circuit diagram of the T4 (ON) and the T5 (OFF) motion detection pathways. Visual input from photoreceptors R1-6 is split into parallel pathways at the level of the lamina. The ONpathway (blue) is shown to involve lamina neuron L1 and at least two postsynaptic cells, Mi1 and Tm3, in the medulla. These cells contact the dendrites of T4 cells. In the OFF-pathway (green), lamina cells $L 2$ and $L 4$ synapse onto medulla neurons Tm1, Tm2 and Tm4. In addition, lamina cell L3 synapses onto Tm9. All four medulla neurons contact the dendrites of T5 cells. Directionally selective signals are carried via T4 and T5 cells to the four layers of the lobula plate where T4 and T5 cells with the same preferred direction converge again on the dendrites of the tangential cells 
(in yellow). Inhibition is conveyed via hypothetical, local interneurons from one layer to the adjacent one (in red) [from 53].

T4 and T5 cells have been speculated to be the output elements of the elementary motion detector based on the anatomical observation that T4 and T5 cells both have four different subtypes that project to four different layers in the lobula plate [21], and the finding that these four lobula plate layers are stained by activity dependent deoxyglucose labelling depending on the direction of a motion stimulus [54]. However, their small size made electrophysiological recordings very difficult, and for years the actual neuronal implementation of the Reichardt detector remained in the dark. This situation changed dramatically only in the last few years with the availability of genetic tools in the fruit fly, Drosophila melanogaster. Specific driver lines target subpopulations of neurons, guided by electron microscopic connectomics studies. This enabled the identification of neuronal components of the motion detection pathway, the description of their functional properties, and the examination of their behavioral significance. Most importantly, T4 and T5 cells have been confirmed as the output elements of the elementary motion detector, T4 being sensitive to moving ON edges, and T5 being sensitive to moving OFF edges. Their terminals in the four different layers of the lobula plate, and hence their four different subtypes, selectively respond to the four cardinal motion directions [55]. Genetically silencing T4 and T5 cells at the same time blocks the electrophysiological response to moving gratings in downstream lobula plate tangential cells [LPTCs; 56] and the behavioral response to grating motion [57]. Silencing either T4 or T5 cells separately abolishes the LPTC response as well as the behavioral response to ON and OFF edge motion, respectively [55]. T4 and T5 cells have thus been established as the outputs of the ON and OFF motion detection pathway.

Electron microscopic studies have elaborated on these pathways' cellular components between L1/L2 and T4/T5. Accordingly, the ON pathway leads from L1 via Mi1 and Tm3 to T4 [58]. The OFF pathway, on the other hand, leads from L2 via Tm1, Tm2, and Tm4 to T5. In addition, T5 receives synaptic input from L3 via Tm9 [52]. Within the OFF pathway, L2, L4 and Tm2 form a cholinergic microcircuit, with the two reciprocally connected $\mathrm{L} 2$ and $\mathrm{L} 4$ both projecting onto $\mathrm{Tm} 2$ [45]. Interestingly, when the 
synaptic inputs of Mi1 and Tm3 on the dendritic trees of T4 cells are traced back to their L1 inputs in the lamina cartridges, a small spatial offset is evident between these inputs and thus, between their anatomical receptive fields. The direction of this offset corresponds with the lobula plate layer the respective T4 cell projects to and hence, its direction selectivity [58]. This arrangement could allow for an implementation of the two arms of an elementary motion detector, one realized by Mi1, the other one by Tm3 neurons. Possibly, the two neuron types could utilize different transmitter-receptor systems, with a fast, ionotropic receptor for the direct, and a metabotropic receptor for the delay line. A comparable spatial displacement could also be found for the Tm1/Tm2 and the Tm2/Tm9 input pairs on the dendrites of T5. However, information about the corresponding projection of T5 onto the lobula plate is missing [52]. On the other hand, a potential molecular mechanism for the multiplicative stage of the Reichardt detector has been proposed for this circuit: $\operatorname{Tm} 1, \operatorname{Tm} 2$ and $\operatorname{Tm} 9$ all have a cholinergic phenotype $[45,52]$, and T5 expresses both nicotinic and muscarinic acetylcholine receptors. Since the inputs of $\operatorname{Tm} 1 / \operatorname{Tm} 2$ and $T m 2 / T m 9$ are spatially segregated on $T 5$ dendrites, this might allow for the implementation of a direct and a delay line and a multiplicative interaction between them, if nicotinic and muscarinic receptors are also asymmetrically expressed on T5 dendrites [52].

The functional significance of some of these circuit elements has already been verified. Cell-type specific calcium imaging confirmed the selectivity of Mi1 and Tm3 for ON stimuli, and of Tm1, L4 and Tm2 [59-61] for OFF stimuli. Furthermore, silencing of either L4 or Tm2 abolishes the response to OFF motion in LPTCs [60]. Interestingly, the electrophysiological responses of Mi1 and Tm3 are temporally slightly offset. Accordingly, the response of $\operatorname{Tm} 1$ is delayed relative to $\operatorname{Tm} 2$. Although this offset is rather small, the authors of this study suggest that it represents the temporal delay between the two arms of a Reichardt detector, and Mi1/Tm3 and Tm1/Tm2 form two elementary motion detectors for ON and OFF stimuli, respectively [61].

The subtractive stage of the Reichardt detector appears to be implemented as an interaction between synaptic excitation and inhibition on the dendrites of tangential cells in the lobula plate $[62,63]$. Recent experiments suggest that while the excitation is likely 
provided directly from T4/T5 cells, the inhibition is conveyed indirectly via intermediate interneurons that receive input from T4/T5 cells with opposite direction selectivity [64]. Excitatory transmission from T4/T5 to the lobula plate is cholinergic $[52,64]$, and sensed by nicotinic acetylcholine receptors on the dendrites of VS cells [65].

\subsubsection{VS cell response properties}

The third visual neuropil, the lobula plate, contains large cells that extend their dendrites perpendicularly to its columnar layout and cover large parts of the neuropil. Calliphora has roughly 60 of these lobula plate tangential cells (LPTCs). They can be categorized according to their electrical response mode. Some LPTCs elicit spikes and respond with a change in their firing rate, others respond only with a graded change in membrane potential, and some, like the VS cells that are studied here, respond with a graded change in membrane potential, superimposed by irregular spike-like events [66] (Fig. 1.7).

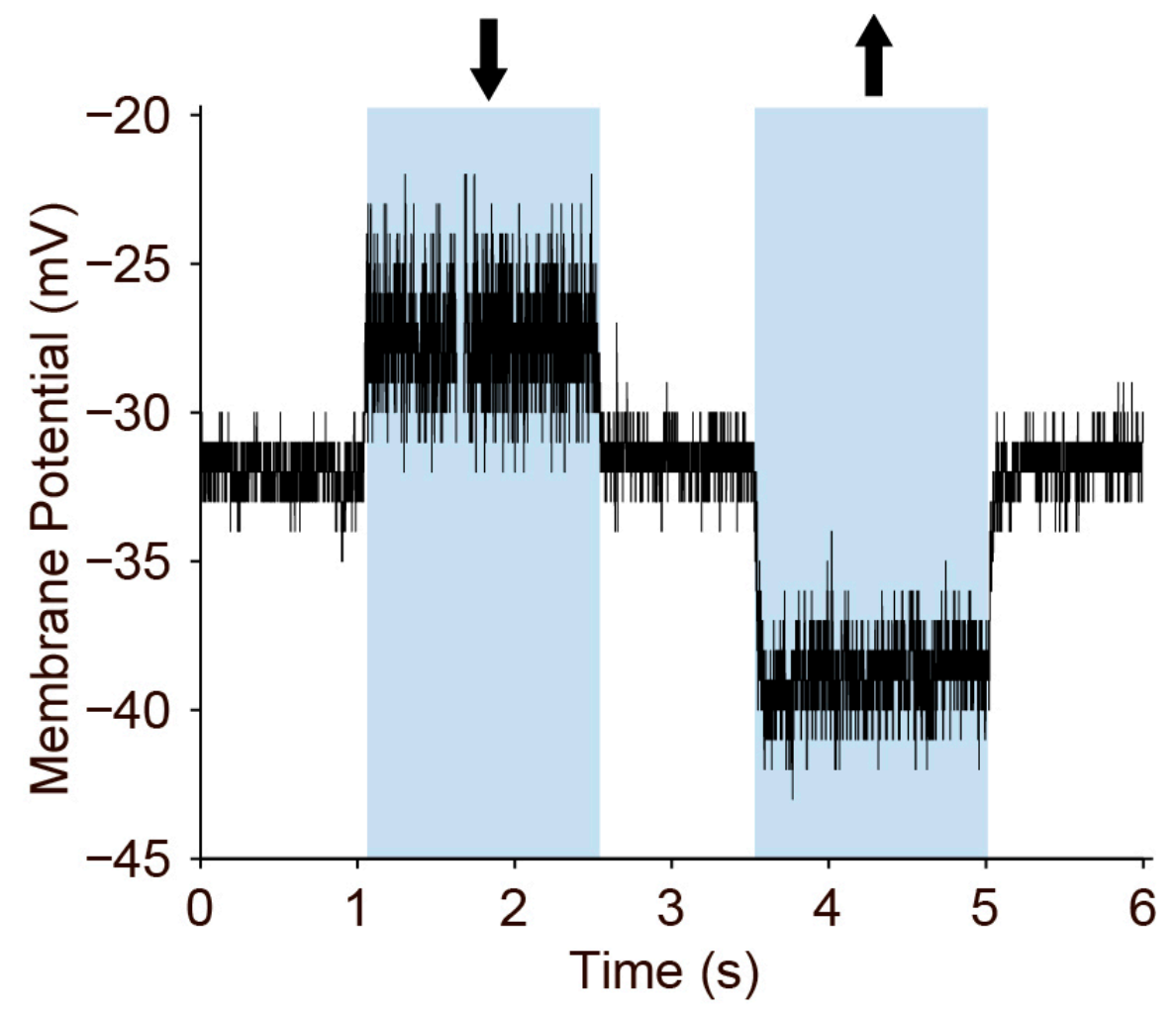

Figure 1.7 Electrophysiological response to motion in a VS cell. Membrane voltage in a VS 4 cell in response to downward (left blue box) and upward (right blue box) motion of a horizontal rectangular grating covering $\sim 30^{\circ}$ along the azimuth and elevation in the frontal visual field. 
Alternatively, LPTCS can be categorized with regard to their direction selectivity. Among the best-studied LPTCs are the horizontal and vertical system cells (HS and VS cells). HS cells depolarize in response to front-to-back and hyperpolarize in response to back-to-front motion. VS cells respond most strongly to vertical motion. For the most part, they depolarize in response to downward and hyperpolarize in response to upward motion. However, upon closer inspection across visual space, their receptive fields are much more complex and diverse. Probing their sensitivity to local motion in small parts of visual space, as has first been done with small, rotating dots [67], reveals broad, complex flow fields with a rich structure. These flow fields match patterns of global motion that are elicited during self-motion of the animal ('optic flow') [for review, see 20].

In the blowfly, VS cells comprise a set of 10 large, T-shaped neurons. Both their dendritic spanning fields, i.e., the tissue area covered by their dendritic arbors $[68,69]$, as well as their receptive fields [70] are highly stereotyped across individuals. Arranged in a row, VS cells are numbered from 1 to 10 from most distal to most proximal [68]. The center of their rotational receptive fields shifts accordingly across the mediolateral axis $[70,71]$, which led to the hypothesis that they act as a set of matched filters for optic flows elicited by rotation of the animal around particular body axes $[72,73]$. For example, the left hemisphere VS 5 will respond best to an optic flow pattern elicited by a rightward roll, i.e., a rightward rotation around the longitudinal body axis. The VS 9 cells in both hemispheres will respond most strongly when the fly performs forward pitch, a rotation around the horizontal body axis (Fig. 1.8).

\subsubsection{VS cell output and behavioral relevance}

Flies can use this information for course control, i.e., to counteract rotations, as they may occur when a wind gust hits the animal during flight and causes a deviation from its course, or to stabilize the image of the world on its retina by head movements. The underlying behavior is called the optomotor reflex. When a tethered fly is placed in a striped, rotating cylinder, it will follow the rotating pattern with a head movement. Walking or flying flies show a similar following behavior [for review, see 20]. LPTCs are crucially involved in the optomotor response. In animals with mutations that affect LPTCs [74] or with experimentally ablated LPTCs $[75,76]$, the optomotor response is severely 
impaired. Notably, these inactivation studies included large sets of LPTCs, or they only tested the yaw response to horizontal pattern motion, which may be relevant only for HS and not VS cells. The pivotal role of HS cells in the optomotor response has also been confirmed by optogenetically triggering yaw responses in Drosophila [77]. However, due to the overall similarity of HS and VS cells, it is generally believed that these conclusions extend to VS cells. 
a

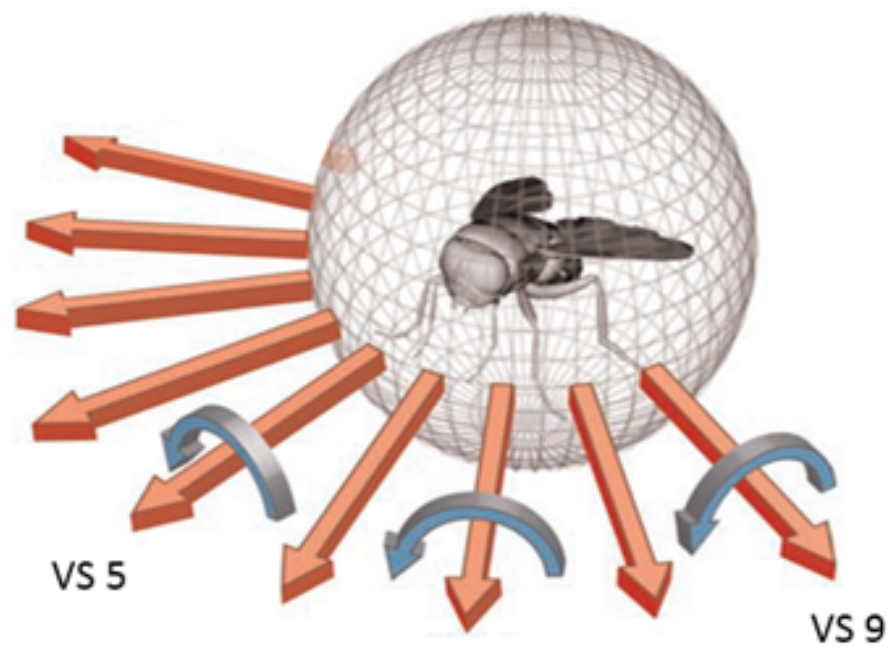

VS 7

b
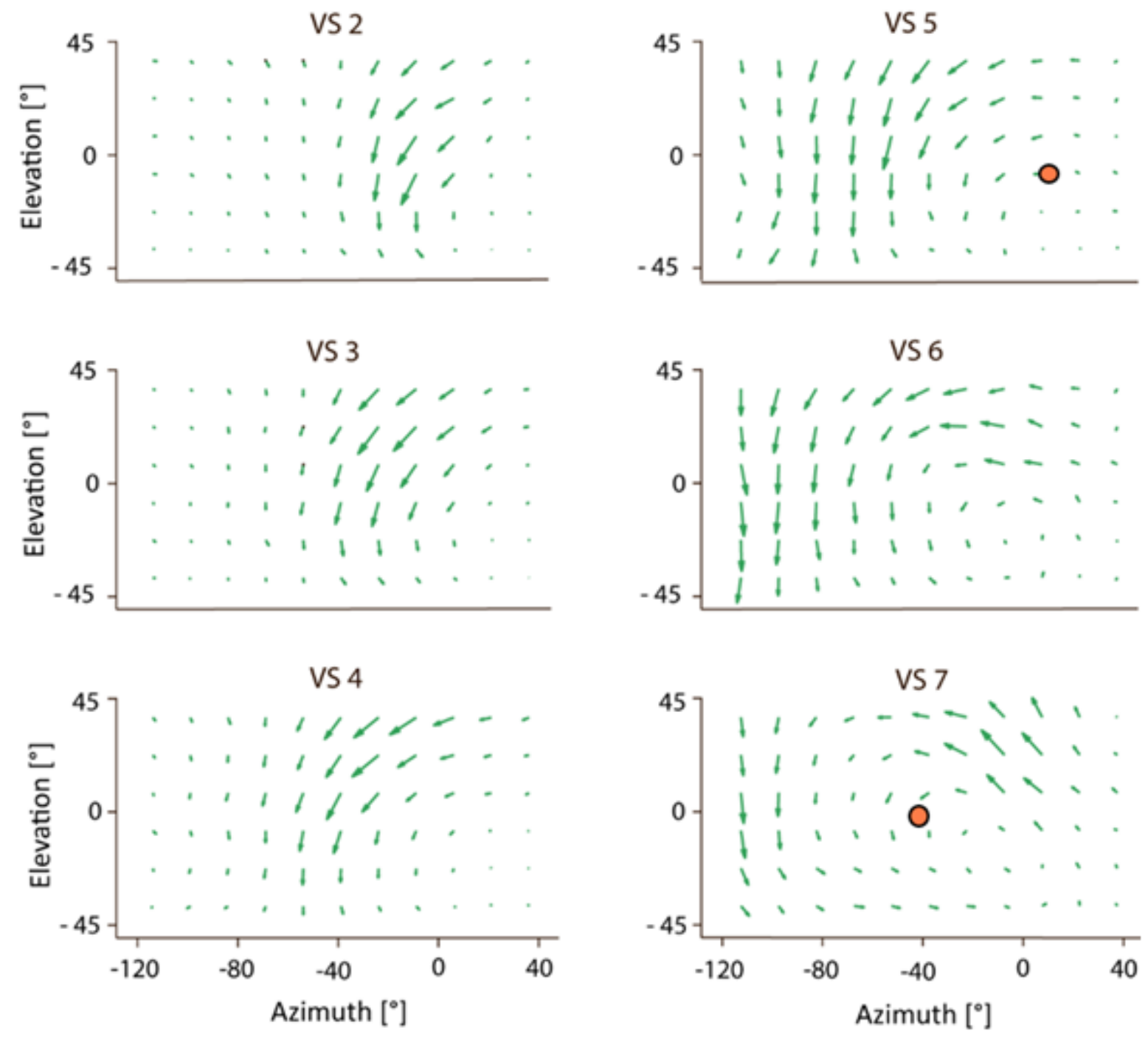

Figure 1.8 VS cell receptive fields resemble optic flows elicited by ego motion. a Schematic illustrating ego rotations of the fly. Red arrows indicate the respective VS cells' preferred axes of rotation, blue arrows indicate the preferred rotation of the fly. Image adapted from [78], which 
again is modified from [79]. b Receptive fields measured electrophysiologically at the axons of VS cells [data from 71, reprinted with permission]. In these vector field plots, the length of an arrow indicates the strength of the local direction tuning, and the direction indicates the local preferred direction. The red dots mark the center of rotation for two of the VS cells illustrated in a.

According to their role in optomotor reflex behavior, VS cells synapse onto descending neurons, which either innervate neck muscles for the control of head movements, or project onto motor neurons in the thoracic ganglion [80-85].

\subsubsection{VS cells in the lobula plate network}

A number of different inputs appear to shape the VS cells' complex receptive fields. In addition to the feedforward input from T4/T5 cells $[55,56]$, three types of lateral inputs from within the lobula plate network have been demonstrated for VS cells. First, all VS cells are connected sequentially via electrical synapses at their axons [86]. Second, proximal and distal VS cells mutually inhibit each other via intermediate interneurons that synapse onto the VS cells' axons [87]. Finally, proximal VS cells (VS 7-10) form dendro-dendritic gap junctions with dCH (dorsal centrifugal horizontal cell)[87] (Fig. 1.9). $\mathrm{DCH}$, on the other hand, does not receive feedforward input from the bushy T-cells on its own. Rather, it receives lateral input from HSN (horizontal system cell north), a lobula plate neuron tuned mainly to horizontal motion, via dendro-dendritic gap junctions [88], and input from the contralateral hemisphere via chemical synapses from the contralateral $\mathrm{H} 1, \mathrm{H} 2$ and $\mathrm{Hu}$ cells. The input from $\mathrm{H} 1$ arrives at dCH's dendrites in the lobula plate, whereas $\mathrm{H} 2$ and $\mathrm{Hu}$ synapse onto a small arborization of $\mathrm{dCH}$ in the protocerebrum, near its axon terminal. These contralateral inputs favor $\mathrm{dCH}$ 's response to optic flows elicited by a yaw rotation over those elicited by forward thrust $[89,90]$. 

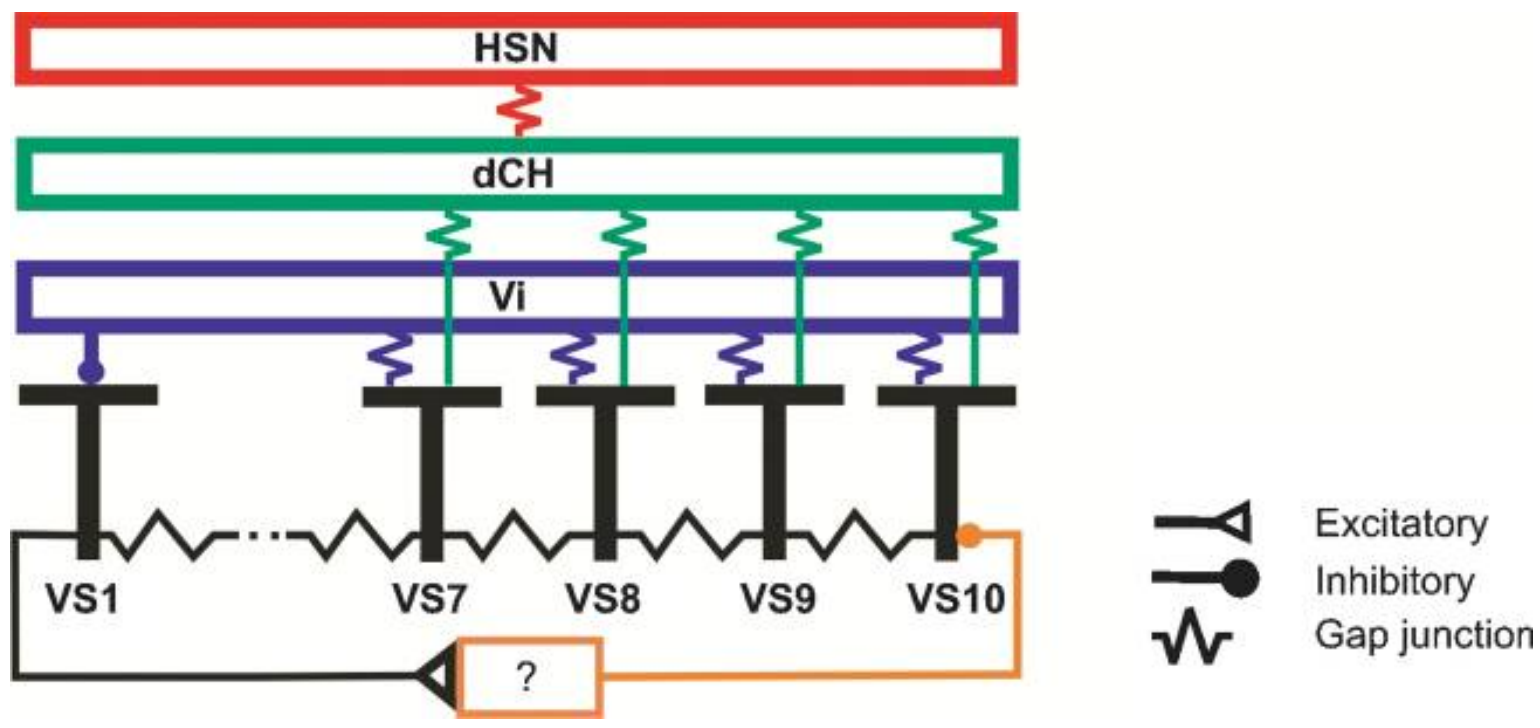

Figure 1.9 Lateral connectivity of VS cells. Circuit diagram illustrating the lateral connectivity of VS cells as described in chapter 1.2.4. Chemical excitatory and inhibitory and electrical synapses are marked as indicated in the legend to the right. The orange box (?) indicates a yet unidentified inhibitory interneuron [from 87].

\subsection{Project question}

What does the subcellular activity of the cells embedded in this intricate network look like, and how do they assemble their optic flow receptive fields? A recent simulation study that takes into account a large body of experimental results argued that the columnar feedforward input may encode pure downward motion in a narrow stripe of visual space, whereas horizontal and upward motion components of the receptive fields are imported via lateral inputs [91]. According to this model, proximal VS cells receive horizontal motion input via the dendro-dendritic coupling to $\mathrm{dCH}$, while their frontal upward motion preference is due to the axonal inhibition from a distal VS cell. The rotational structure of the VS cells' receptive fields would thus be produced by network interactions, and would not be completed until the level of their axons. Alternatively, rotational receptive fields may already be assembled by the appropriate selection of local motion inputs on the dendritic level. 
Early calcium imaging studies using full field illumination and CCD camera imaging provided evidence for a topographical organization on the dendrites of HS and VS cells. When gratings of $\sim 20^{\circ}$ in diameter were presented at two different elevations, a dorsal and a ventral area of an HS cell lit up, respectively [92, 93]. Sweeping a bar across the visual field of the fly lead to sequential activation of the dendrite along the dorso-ventral axis of a VS 1 cell [94]. Another study examined the direction tuning in subregions of selected VS cells in response to a global motion stimulus, but did not provide information about its sensitivity to local motion responses [95].

Thus, a thorough characterization of VS cell receptive fields that ascribes local direction selectivity with a high spatial resolution across visual space has only been measured electrophysiologically, representing axonal output $[67,70]$. Such a detailed analysis is still missing on the dendritic input level. In this thesis, I examine both the preferred location and the preferred direction of local motion cues for fine branchlets across the entire dendritic arborizations of a set of VS cells. Beyond the scope of the fly visual system, this is, to my knowledge, the first study that maps both stimulus space and stimulus feature onto the dendritic tree of individual neurons, thereby providing a comprehensive picture of the cells' input activity that can be related to their axonal output.

\subsection{2-photon imaging}

For this endeavor, CCD camera imaging is not sufficient. The technique lacks spatial resolution and does not allow for a reliable measurement of calcium signals in fine dendritic branches, due to difficulties with background subtraction [as discussed in 92 , 96]. I therefore used 2-photon imaging [97], which offers several advantages for in vivo imaging. The key advantage is that excitation quadratically depends on light intensity, and light intensity decreases dramatically outside of the focused laser beam. Therefore, excitation occurs almost exclusively in the focal volume, and practically all collected photons originate from the focal volume, scattered or unscattered. This improves the signal to noise ratio, especially for highly scattering tissues or when imaging deeper in the tissue. As a consequence, the effective spatial resolution of a 2-photon microscope can be 
superior to that of a confocal microscope, although roughly twice the excitation wavelength is used, and one would expect a spatial resolution of 2-photon imaging that is worse by about a factor of two compared to confocal imaging. However, this would only be true theoretically, for an infinitely small pinhole. In practice, there is a trade-off in the size of the pinhole between the achieved spatial resolution and the amount of collected photons [98].

Furthermore, using longer wavelengths for 2-photon excitation provides several other advantages that can be even more important when imaging in vivo: (1) reduced autofluorescence, which again improves the signal to noise ratio and reduces phototoxicity, (2) far less photobleaching of the fluorescent dye outside of the focal volume, which is critical for long in vivo experiments at different depths in the tissue, (3) no interference with the visual system, since the excitation wavelengths used are outside of the absorption spectra of the fly's photoreceptors (Fig. 1.10), and (4) superior depth penetration $[98,99]$.

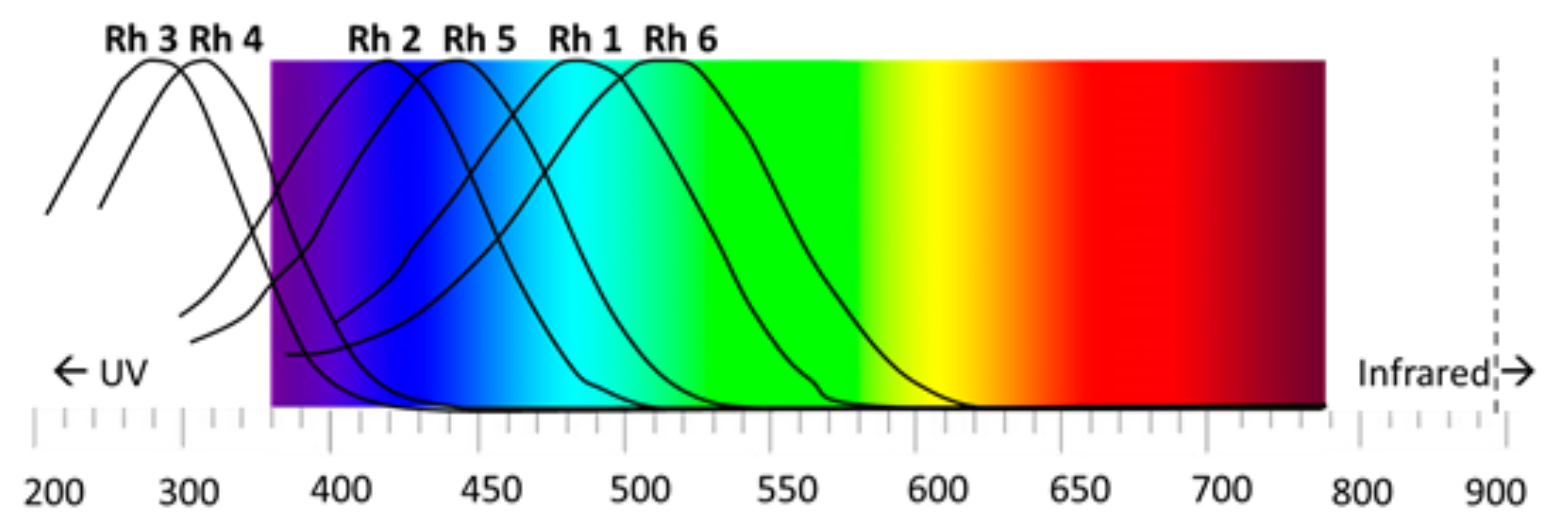

Figure 1.10 Laser excitation does not interfere with the visual system. Absorption spectra of the different rhodopsin types in the retina of Drosophila [adapted from 100]. Dashed line marks wavelength used for 2-photon excitation. 


\section{Materials and Methods}

\subsection{Fly Preparation}

Blowflies (Calliphora vicina, 2-7 days old, laboratory stock, of either sex) were briefly anesthetized with $\mathrm{CO}_{2}$ and fixed with wax to a small custom made plastic holder. The fly's legs were removed and its wings and abdomen were immobilized with wax. To prevent motion artifacts caused by peristalsis, the proboscis was cut and the esophagus was removed from the head. The head capsule was opened from behind and tracheae, air sacs and fat capsules were removed.

VS cells in the left hemisphere were filled with a calcium indicator through sharp microelectrodes. Electrodes were pulled on a Flaming/Brown micropipette puller (P-97; Sutter Instruments) from glass capillaries (GB100F-10; Science Products GmbH). The tip of the electrodes was filled with 5 mM Oregon Green Bapta-1 (OGB-1; Molecular Probes; a chemical calcium indicator with relatively high calcium affinity; $\mathrm{K}_{\mathrm{d}}=170 \mathrm{nM}$ determined by the manufacturer in vitro at $22^{\circ} \mathrm{C}$ in $100 \mathrm{mM} \mathrm{KCl}, 10 \mathrm{mM}$ MOPS, $\mathrm{pH}$ 7.2) solution. The shaft was filled with a $2 \mathrm{M} \mathrm{KAc}$ and $0.5 \mathrm{M} \mathrm{KCl}$ solution.

\subsection{Stimulation setup and 2-Photon Microscopy}

Visual stimuli were displayed on a custom-built cylindrical LED display [18] that covered $\sim 90^{\circ}$ of the vertical and $\sim 180^{\circ}$ of the horizontal visual field of the fly, centered with respect to the elevation in front of the fly, and ranging from about $-130^{\circ}$ to $+50^{\circ}$ along the azimuth. It allows refresh rates of up to $600 \mathrm{~Hz}$ with 16 intensity levels, ranging from 0 to $70 \mathrm{~cd} / \mathrm{m}^{2}$ with a spectral peak at $568 \mathrm{~nm}$.

For calcium imaging I used a custom built two-photon laser scanning microscope [97] consisting of the following components: a diode-pumped Ti:Sapphire laser (Mai Tai; Spectraphysics), a Pockels cell (350-80; Conoptics), scan mirrors (6215; Cambridge Technology), a scan lens (4401-302; Rodenstock), a tube lens (MXA22018; Nikon), a dichroic mirror (789 DCSPR 25.5x36; AHF Tuebingen), and a 40x water immersion objective (440095; 
Zeiss, NA=0.8). The objective can be moved along all three axes by a step-motor driven micromanipulator (MP 285-3Z; Sutter Instruments), while the specimen is held still. A 2-photon excitation wavelength of $890 \mathrm{~nm}$ was used. Emitted fluorescence light was collected by a photomultiplier tube (PMT, R63570; Hamamatsu). A telescope built from two curved mirrors was used for beam expansion. Two flat mirrors could be switched into the laser path to circumvent beam expansion. This allowed me to use two beam sizes: one overfilling the objective, yielding a high z-resolution for morphology stacks, and another one slightly underfilling the objective, sacrificing z-resolution for depth of view. I took advantage of the latter during functional calcium imaging to simultaneously measure multiple dendritic branchlets located at different depths.

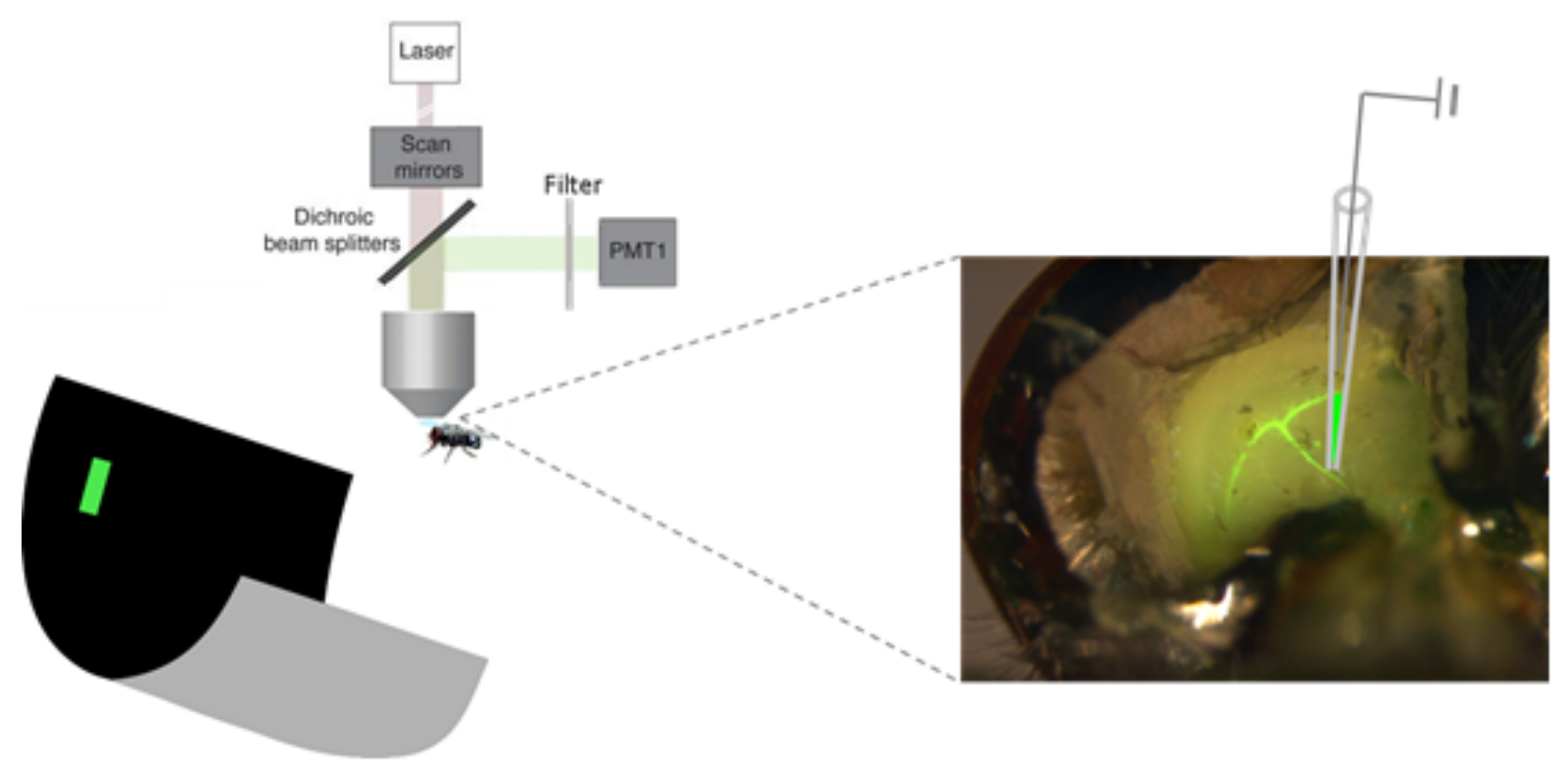

Figure 2.1 Setup for 2-photon imaging under visual stimulation. The fly is presented with visual stimuli on a cylindrical LED display (bottom left) during 2-photon imaging from above (top left). The image on the right shows a photograph of a VS 4 cell in the left hemisphere filled with OGB-1 through a sharp microelectrode (overlay of a bright field image and a fluorescence image). 


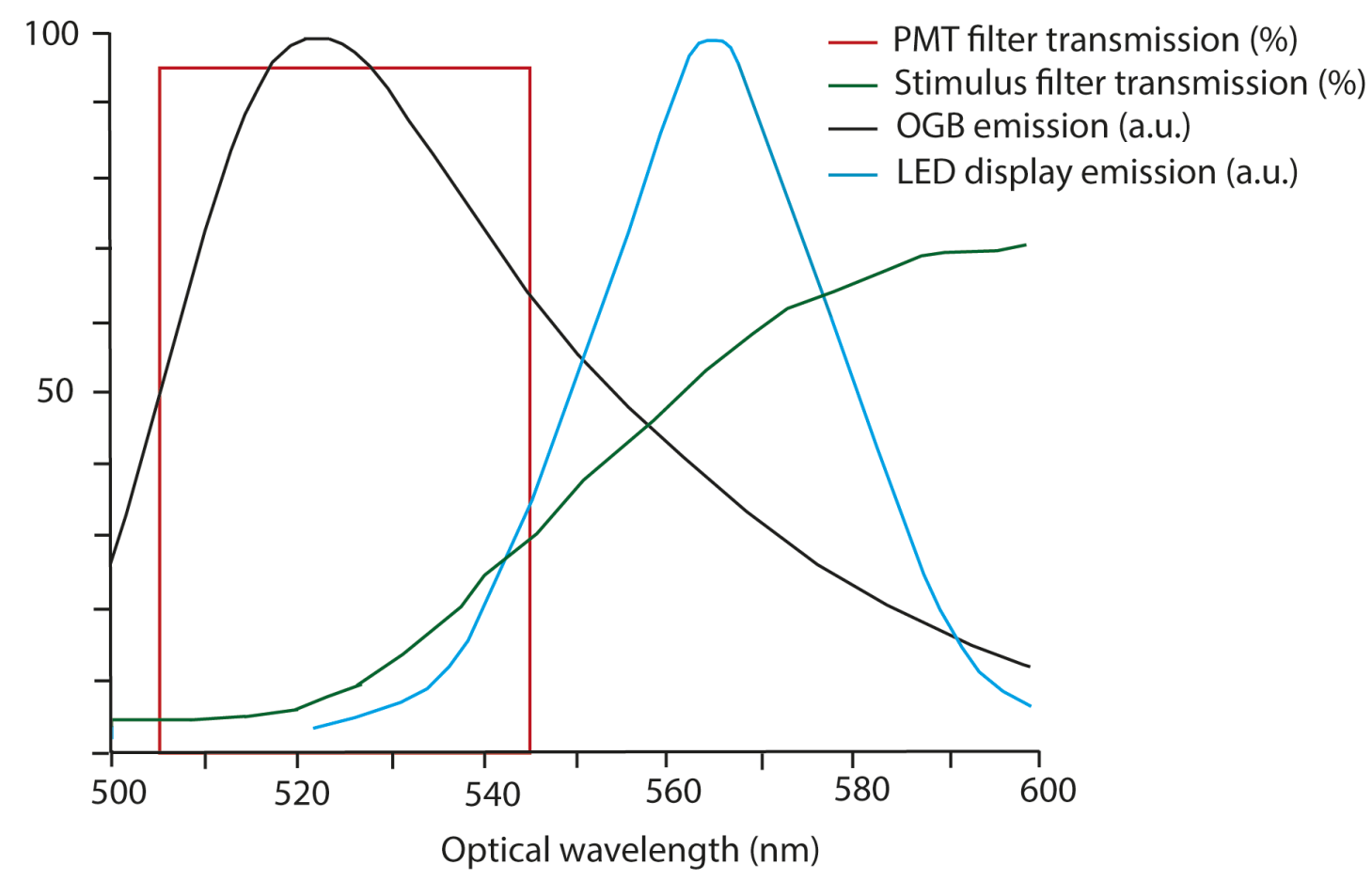

Figure 2.2 Spectral separation of stimulus light and fluorescence signal. Emission spectra of OGB-1 and the LED display and spectra of the filter in front of the PMT and the LED display, respectively, are shown as indicated in the legend to the right.

The spectrum of the LED display and the calcium dye overlap to a great extent (turquoise and black line, respectively, Fig. 2.2). This leads to a strong contamination of the fluorescence measurement by stimulus light. I pursued several potential solutions to this problem. A previous study [101] interlaced stimulus presentation and laser scanning in such a way that the stimulus was shown only during a $0.4 \mathrm{~ms}$ retrace period of the scan mirror, while during each $1.6 \mathrm{~ms}$ line scan, no stimulus was shown. With a flicker fusion frequency of around $250 \mathrm{~Hz}$ for Calliphora vicina [100], the fly can be assumed to perceive this $500 \mathrm{~Hz}$ flicker as a continuous stimulus, albeit at a luminance reduced to $25 \%$. However, this solution did not work for my setup, since the PMTs saturated upon stimulus presentation, and recovered at a timescale of hundreds of milliseconds. This markedly reduced PMT sensitivity during a subsequent line scan and introduced dynamic artifacts if a flickering stimulus was presented. Another solution I considered was to use shutters in front of the PMTs. However, with opening and closing times in the millisecond range, mechanical shutters are too slow for my purpose. Optical shutters, on the other hand, would be fast enough, but have an open state transmission of only $25-30 \%$ and thus take away too much 
of the fluorescence signal. A circuit diagram to gate the power supply to the PMTs on a sub millisecond timescale was suggested by Hamamatsu, but neither our electronics workshop nor Hamamatsu themselves were able to successfully implement it. Ultimately, I settled on a combination of shielding and spectral separation, which successfully avoided contamination by stimulus light. A black PVC foil extended from the fly holder over the LED stimulus display (Fig. 2.3). The fly head was surrounded by an upside down cone of black aluminum foil, leaving only a small window to measure fluorescence emitted from the brain, but at the same time allowing for a maximal lateral field of view for the animal. Leaking stimulus light was spectrally separated from OGB fluorescence by two filters. First, the LED display was covered with a UV filter that blocks wavelengths below $550 \mathrm{~nm}$ (ASF SFG 10; Microchemicals) (Fig. 2.2 and 2.3). This also reduced the maximum stimulus luminance to $33 \mathrm{~cd} / \mathrm{m}^{2}$. Second, a band-pass filter (525/40, F37-524; AHF Tuebingen) was inserted before the PMT (Fig. 2.2).

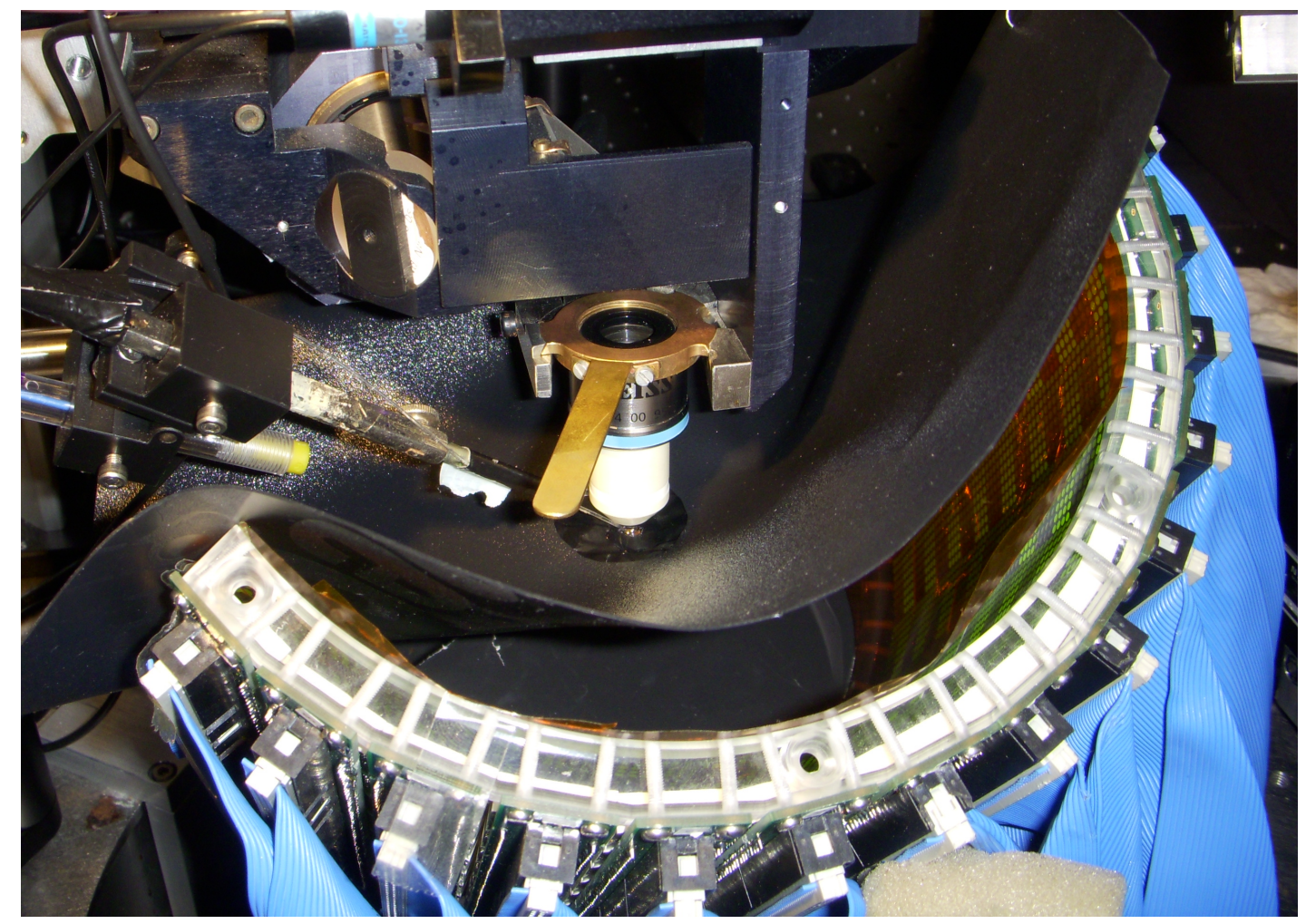

Figure 2.3 Shielding of stimulus light. Photograph of the setup from above. The black plastic foil and the black aluminum foil around the fly's head shield stimulus light emitted from the LED display. 
The system was controlled with the MATLAB-based open-source software Scanlmage [102] (version r3.7). For functional calcium imaging, images were acquired at a resolution of $128 \times 128$ pixels and a frame rate of $7.81 \mathrm{~Hz}$. For anatomical reconstructions, several z-stacks, covering the entire cell or only its dendritic tree, were acquired with an xy-resolution of $512 \times 512$ pixels $(0.3 \mu \mathrm{m})$ and a z-resolution of $2 \mu \mathrm{m}$. Functional imaging locations were mapped onto anatomical reconstructions by taking into account changes of the respective hardware and software settings in the Scanlmage software, i.e. the angular range of the scan mirrors, the zoom factor, the scan rotation, and the image resolution.

\subsection{Visual stimuli and data analysis}

Z-stacks were reconstructed using the software package Amira (version 5.3.1; Mercury Computer Systems, Berlin). VS cell types were identified based on the position of their ventral dendrite relative to the borders of the lobula plate [103].

Custom-written scripts in MATLAB (version 7.12.0) were used for programming visual stimuli as well as for analysis of the functional imaging data. Frames were detected as motion artifacts and linearly interpolated if their 2-D correlation with an average picture computed over the surrounding 100 frames was below a preset threshold. The threshold was manually set depending on drift and noise level in each experiment. This method was employed for only a few, brief periods in a given trial. Experiments with frequent motion artifacts were completely discarded. Fluorescence was averaged in manually defined regions of interest (ROIs) that typically enclosed fine dendritic branchlets of $\sim 1-5 \mu \mathrm{m}$ in diameter. Fluorescence traces were corrected for drift by subtraction of a 300 frame moving average window, converted to $\Delta F / F$ using a 20 frame interval in the beginning of each trial as baseline, and averaged across trials. Averaged $\Delta F / F$ traces were smoothed with a Gaussian kernel (standard deviation 2 frames, i.e. $256 \mathrm{~ms}$ ) and deconvolved according to:

$$
d F / F_{\text {deconv }}(t)=\tau \cdot(d F / F(t)-d F / F(t-1))+d F / F(t)
$$

The time constant, $\tau$, was estimated in each cell individually. To this end, the impulse response of the system was measured: a round patch of $21^{\circ}$ in diameter filled with a square wave grating of $12^{\circ}$ spatial wavelength was moved at a temporal frequency of $5 \mathrm{~Hz}$ for 2 
scan frames, i.e. $256 \mathrm{~ms}$, and a first order low pass filtered step function was fitted to the $\Delta \mathrm{F} / \mathrm{F}$ response of a fine dendritic branch. This was done for only one branchlet per cell after multiple experiments in different VS cell types had shown that time constants were always comparable across the dendritic tree. The average $\tau$ was $3.3 \pm 1.6 \mathrm{~s}(\mathrm{n}=24$ cells, mean $\pm s d)$.

Receptive fields were measured as follows: Small bars $\left(18^{\circ}\right.$ long, $4.5^{\circ}$ wide, with a luminance of $33 \mathrm{~cd} / \mathrm{m}^{2}$ on a dark background) were moved across the whole LED arena at a constant velocity of $14 \%$ s. I showed horizontal bars which moved upward or downward at 10 different azimuthal angles, and vertical bars which moved left or right at 5 different elevations, spaced by $18^{\circ}$. In this way, the entire LED arena was covered by the stimulus in a $5 \times 10$ grid (Fig. 3.1a). Trials with the four different directions were randomized. Each direction was shown twice. The deconvolved $\Delta F / F$ responses to horizontally moving bars at the 5 different elevations were binned and averaged in 10 bins along the azimuthal direction. Accordingly, responses to vertical motion at 10 different azimuthal angles were binned and averaged in 5 bins along the elevation direction. As such, a $5 \times 10$ response matrix was obtained for each motion direction. Negative entries were considered noise and rectified to zero, since I never observed consistent fluorescence decreases in responses to visual stimulation. The responses to the four directions in each grid field were then multiplied with the respective unit vectors pointing in the direction of the stimulus and summed to obtain the preferred direction vector for that field. Direction selectivity indices (DSIs) were calculated as

$$
D S I=\frac{\left|\sum_{i=1}^{4} r_{i} \vec{e}_{i}\right|}{\sum_{i=1}^{4} r_{i}}
$$

where $r_{i}$ is the response to motion in the direction of unit vector $\vec{e}_{i}, D S I \in[0,1]$.

Stimulus size and velocity were chosen as a compromise between resolution of visual space and coverage of the dendritic tree of each cell. On the one hand, I aimed at covering visual space with a grid as fine as possible. The smaller the bar stimulus, the finer the grid, but the longer an individual trial takes. At the same time, there is an upper limit on the velocity of the bars that still yields a good signal-to-noise ratio at a scanning frame rate of $7.81 \mathrm{~Hz}$. On the other hand, I wanted to cover the dendritic tree of each cell as exhaustively as possible. Here again I faced a trade-off between the dendritic area covered in one single 
trial (with a lower zoom factor), and signal quality (which increases with the zoom factor). To balance these interests, I chose a 10 by 5 stimulus grid spanning my LED display, which corresponds to a grid field size of $18 \times 18^{\circ}$, and imaged up to 22 separate sections of the dendrite per neuron. A typical experiment took between 4 and 8 hours, including preparation of the fly brain, functional measurements and acquisition of z-stacks for anatomical reconstructions. The actual duration of an experiment depended on the lifetime of the animal.

To validate the receptive field mapping, I first presented small patches of $21^{\circ}$ in diameter that were filled with a square wave grating of $12^{\circ}$ spatial wavelength moving leftward at a temporal frequency of $5 \mathrm{~Hz}$ for 2 laser scan frames, i.e. $256 \mathrm{~ms}$. I showed the grating patch at 10 different azimuthal angles at the preferred elevation of a branchlet. Responses were evaluated as $\Delta F / F$, and enough time was allowed for the fluorescence signal to decay back to baseline between stimulus presentations. The resulting spatial response profile was compared to the binned and averaged deconvolved responses to a small bar moving leftward along the azimuth, again at the branchlet's preferred elevation. The experiment was repeated accordingly with downward moving gratings placed along the elevation and bars moving downward (exemplified in Fig. 3.1d, g). Secondly, I presented the same grating patch at the preferred azimuth and elevation position of the branchlet and moved the grating in 8 different directions. Again, responses were evaluated as $\Delta F / F$. The preferred direction was calculated by vector summation and compared to the preferred direction at the corresponding azimuth and elevation that was determined by my receptive field mapping method (Fig. 3.1k, also see Results, pp. 33-35).

The cylindrical LED display had to be rotated away from a position perpendicular to the fly's azimuthal plane to enable stimulation across the entire dendritic trees of all considered VS cells. Consequently, the regular stimulation grid appeared to some extent irregular and distorted in the perspective of the fly. For an accurate assignment of an azimuth and elevation angle to each field on the grid, I constructed a computer model of the LED cylinder, which virtually reproduced the setup. The LED display was modeled as a cylinder sitting in a coordinate system with the fly at its origin. The cylinder was then rotated and translated using a custom written GUI until the positions of a number of reference points on 
the cylinder relative to the fly matched those that were measured in the actual setup. The deviation of this rotated stimulation grid from a positioning perpendicular to the fly's azimuthal plane was then read out from the model for each grid field (Fig. 2.4).

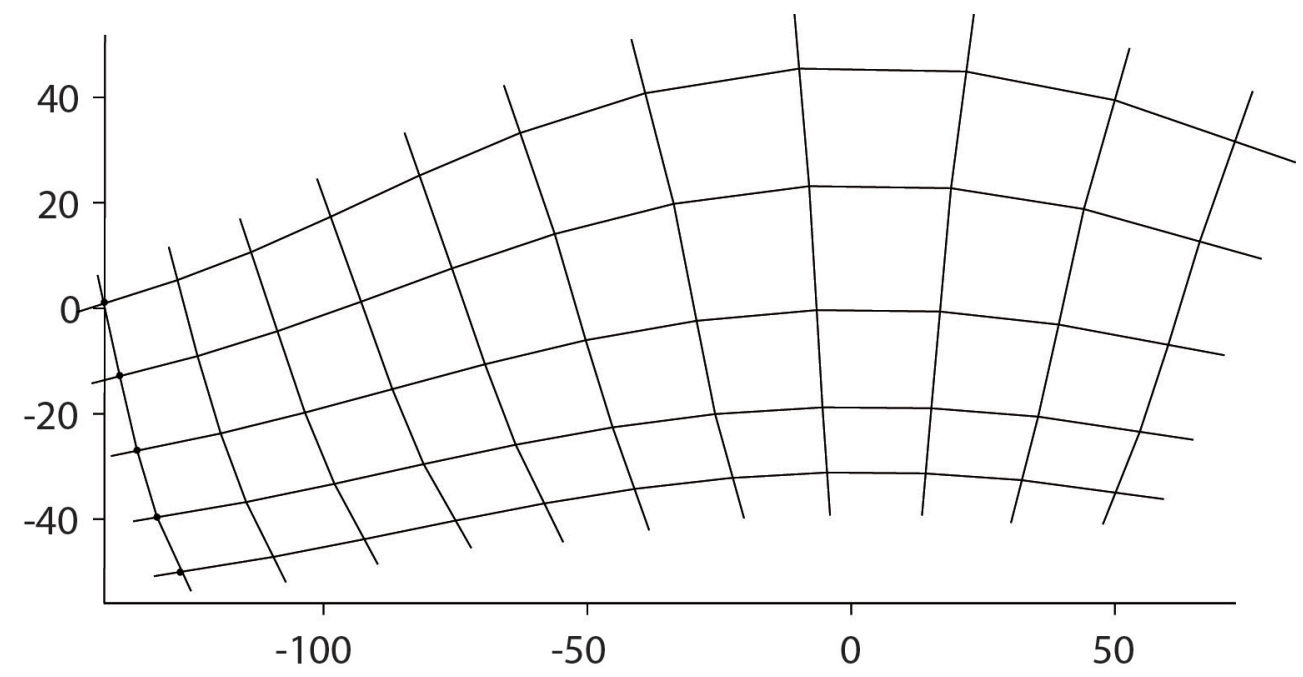

Figure 2.4 The LED display in fly visual coordinates. The cylindrical LED display appears distorted in the perspective of the fly, because it had to be rotated away from a position perpendicular to the fly's azimuthal plane.

The local preferred direction (PD) vectors were corrected by the respective deviation angle to accurately reflect those perceived by the fly. From the fly's perspective, some of the axes of the stimulation grid were not exactly orthogonal. The potential error in PD calculation by vector summation was calculated to be within a range of $0^{\circ}$ and $14^{\circ}$.

For the analysis presented in Fig. 3.4, the grid field with the maximum PD vector length was evaluated as a branchlet's preferred stimulus location. Branchlet coordinates of different VS cells were transferred to a shared lobula plate coordinate system. They were first normalized to the coordinates of the main dendritic bifurcation of the individual cell. Then, they were shifted by the coordinates of the main bifurcation of the respective cell type in a reference set where all VS cells were stained in one animal (depicted in Fig. 3.4a). For contour plots (Fig. 3.4e,f), I used the MATLAB-based tool gridfit [104, http://www.mathworks.com/matlabcentral/fileexchange/8998] to approximate smooth 
surfaces to the preferred azimuth and elevation, respectively, as a function of a branchlet's position in the lobula plate.

For the analysis presented in Fig. 3.6c,d, the PD at the grid field with the maximum PD vector length was selected as a branchlet's main preferred direction. To compare the summed dendritic receptive field $\left(R F_{\text {dend }}\right)$ and the axonal output receptive field $\left(R F_{a x o n}\right.$, Fig. 3.7), the vector fields of all branchlets were normalized to their respective maximum vector length and then summed over all cells of one particular cell type. This cell type averaged $\mathrm{RF}_{\text {dend }}$ was then interpolated to the coordinates of $\mathrm{RF}_{\mathrm{axon}}$. Both $\mathrm{RF}_{\text {dend }}$ and $\mathrm{RF}_{\text {axon }}$ were normalized to their respective maximum vector length.

\subsection{Immunostaining}

VS cells (VS 4 and 7 in separate animals) in 3-5 day old flies were filled with Alexa 488 (Molecular Probes) using sharp microelectrodes. Brains were prefixed for 30 minutes in the intact animal with 4\% PFA (4\% paraformaldehyde in PBS) and then dissected. Dissected brains were fixed for another 30 minutes in 4\% PFA with $0.1 \%$ Triton, washed in PBT $(0.3 \%$ Triton in PBS) and blocked in $10 \%$ goat serum in PBS. The primary antibodies were incubated for $72 \mathrm{hrs}$, the secondary antibodies for $48 \mathrm{hrs}$, washing in PBT in between. After the secondary incubation, brains were washed first in PBT, then in PBS, and embedded in low melting agarose (Serva Electrophoresis GmbH; Heidelberg, Germany). Antibodies used were: Anti-Alexa Fluor 488 Rabbit IgG (Molecular Probes; cell staining) and 4F3 anti-discs large (Developmental Studies Hybridoma Bank; neuropil staining). Sections of 60 or $80 \mu \mathrm{m}$ thickness were cut from the agarose block with a Leica VT 1000S vibratome and mounted in Vectashield (Vector Laboratories, Burlingame, CA). Tissue sections were imaged with a Leica SP5 confocal microscope using a $40 x$ oil immersion objective. Images were taken at a resolution of $1024 \times 1024$ pixels at $1 \mu \mathrm{m}$ intervals.

For the VS cell reference set (Fig. 3.4a), a fly brain was stained with Richardson's stain, cut in $1 \mu \mathrm{m}$ thick sections, and imaged with bright field microscopy. VS cells were subsequently reconstructed using the software package Amira (version 5.3.1; Mercury Computer Systems, Berlin). 


\section{Results}

\subsection{Mapping the receptive fields of small dendritic branchlets}

To measure the subcellular input topography of VS cells, I performed 2-photon calcium imaging in response to visual stimulation of fine branchlets across the entire dendritic trees of individual VS cells. Individual neurons were filled with the calcium indicator Oregon Green Bapta-1 (OGB-1) through intracellular recording electrodes. Previous studies have found two sources for calcium entry in VS cells $[105,106]$. First, insect nicotinic acetylcholine receptors (nAChRs) are calcium permeable. In VS cells, they are most abundant on the fine branchlets of higher order dendrites [65], where the density of postsynaptic sites is highest [107]. Second, VS cells have been shown to contain voltage-gated calcium channels (VGCCS) [106]. The signals I measure here thus reflect direct excitatory input and local deflections in membrane potential that result from the integration of excitatory and inhibitory inputs. A three-dimensional stack of the cell was assembled at the end of each experiment for anatomical reconstructions.

For functional imaging I presented motion stimuli on a cylindrical LED display spanning $\sim 180^{\circ}$ of the fly's visual field along the azimuth and $\sim 90^{\circ}$ along the elevation. I focused on those cells whose receptive fields were covered by my stimulus device, i.e., VS 2-7. VS 1, for example, has a large receptive field that extends to $180^{\circ}$ in the back of the fly [70], an area which was not stimulated by my device. Responses were evaluated based on the relative fluorescence change $\Delta F / F$ in a manually drawn region of interest (ROI). ROIs were chosen to include fine dendritic branchlets, typically of 1-5 $\mu \mathrm{m}$ in diameter. To map receptive fields, I modified a stimulus previously used in electrophysiological experiments [71, 108]. Small, bright bars were moved horizontally across the LED display at 5 elevations, and vertically at 10 azimuthal angles (Fig. 3.1a). I binned and averaged responses to horizontal and vertical bar motion in 10 and 5 bins, respectively, to obtain responses for each of the four motion directions at each location on the $5 \times 10$ grid. Local preferred directions could then be calculated by vector summation (also see Materials and Methods, pp. 26-27). 
a
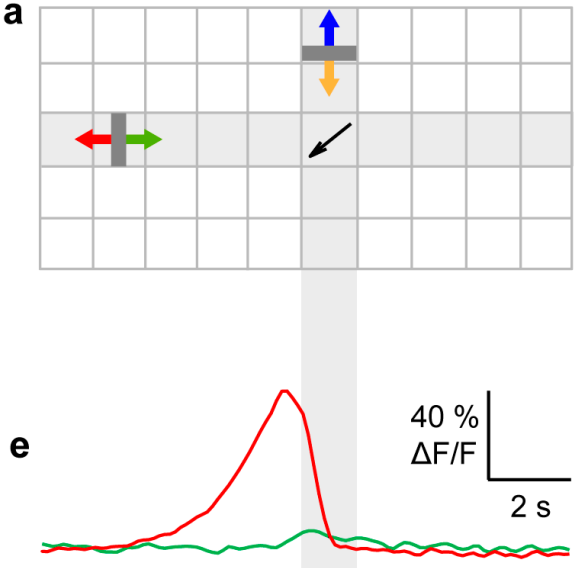

g

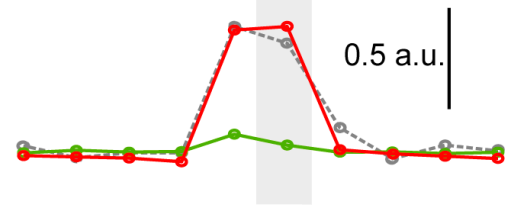

b

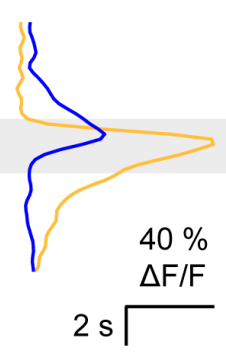

C

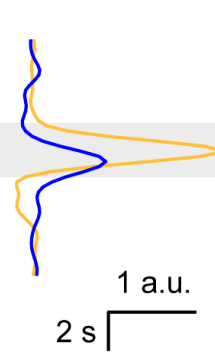

h

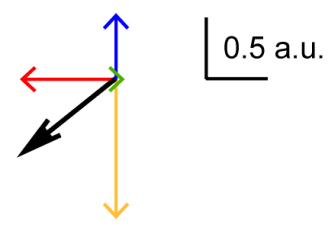

j

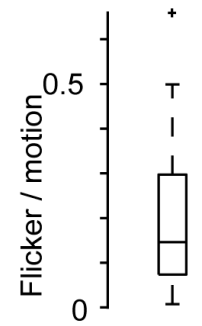

d

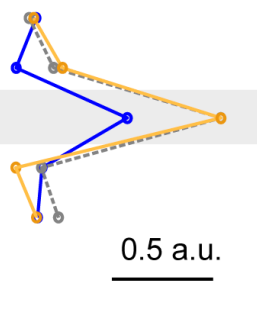

i

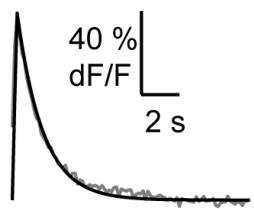

k

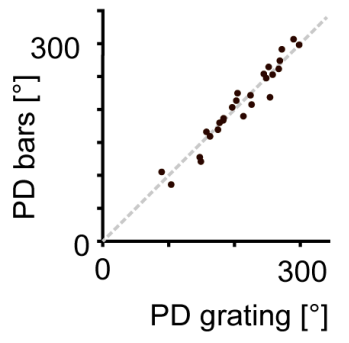

Figure 3.1 Mapping the receptive fields of small dendritic branchlets. a Schematic illustrating the small moving bar stimulus and receptive field calculation. Panels $\mathbf{b}-\mathbf{h}$ demonstrate the calculation of the local preferred direction (black arrow in a) for the grid field at the intersection of the gray shaded rectangles. $\mathbf{b}, \mathbf{e} \Delta \mathrm{F} / \mathrm{F}$ responses to a small bar moving in the indicated directions (red, blue, green and yellow arrows in panel a) across the LED display at the positions marked by the gray shaded rectangles. $\mathbf{c}, \mathbf{f}$ Same as in $\mathbf{b}, \mathbf{e}$ after deconvolution. $\mathbf{d , g}$ Same as in $\mathbf{c , f}$ binned and averaged (colored, solid lines); gray, dashed lines: spatial response controls using moving gratings restricted to small patches along the elevation or azimuth. $\mathbf{h}$ Illustration of the vector summation for the example grid field. Its local preferred direction (black arrow) is calculated from the data points in the gray shaded rectangles in $\mathbf{d}$ and $\mathbf{g}$. $\mathbf{i}$ Estimation of the system's impulse response. $\Delta \mathrm{F} / \mathrm{F}$ response to a small patch of grating at the branchlet's preferred location moving briefly in its preferred direction (gray trace) and parametric fit (black trace). $\mathbf{j}$ Responses of dendritic branchlets of all analyzed VS cells to the appearance of a small stationary grating at their preferred location relative to the response to grating motion. The boxplot indicates median, 25th and 75th percentile. An outlier is marked by the + sign. $\mathbf{k}$ Local preferred directions of dendritic branchlets at their optimal response location determined by the receptive field mapping method illustrated above compared to the local preferred directions determined with a small grating moving in 8 directions. The gray dashed line indicates identity. The difference between local preferred directions measured with the two methods is $-1.3 \pm 14.9^{\circ}$, mean $\pm s d$, which is not statistically different from zero in a one-sample $t$ test, $p=0.67, n=26$ branchlets in 5 cells 
However, a challenge arises in this calculation because the indicator acts as a low pass filter on the actual calcium dynamics [109]. When a bar moves through the receptive field of a branchlet in its preferred direction, the fluorescence signal rises sharply, but decays slowly back to baseline (see for example Fig. 3.1b, yellow trace: response to a downward moving bar). Basing the receptive field calculation on the raw $\Delta F / F$ signal (Fig. 3.1b,e) would thus lead to an overestimation of the motion response at each subsequent location along the bar trajectory. I therefore deconvolved the signal to recover the original calcium dynamics. To this end, I estimated the impulse response of the system by presenting a small patch of a grating in the receptive field of a branchlet and applying a brief motion pulse in the branchlet's preferred direction (Fig. 3.1i). All $\Delta F / F$ traces were then deconvolved accordingly (Fig. 3.1c,f; see Materials and Methods), and the deconvolved traces were binned and averaged as outlined above (Fig. 3.1d,g). Local preferred directions were calculated by vector summation of the binned responses to the four directions of motion (exemplified in Fig. 3.1h for the grid field at the intersection of the vertical and horizontal gray shaded rectangles in Fig. 3.1a).

I performed two controls to validate this receptive field mapping method. First, I tested whether the spatial response profile of the measured branchlet is accurately reflected in the deconvolved $\Delta F / F$ response to a bar moving across the LED display. To this end, I compared it to the $\Delta F / F$ response to moving square wave gratings within small patches, which were consecutively placed at different positions along the respective bar trajectory. Both methods lead to almost identical spatial profiles (Fig. 3.1d,g, compare the red and the yellow trace, respectively, with the gray control traces). I repeated this experiment in a total of 17 branchlets in 4 different cells and obtained similar results (Fig. 3.2). 

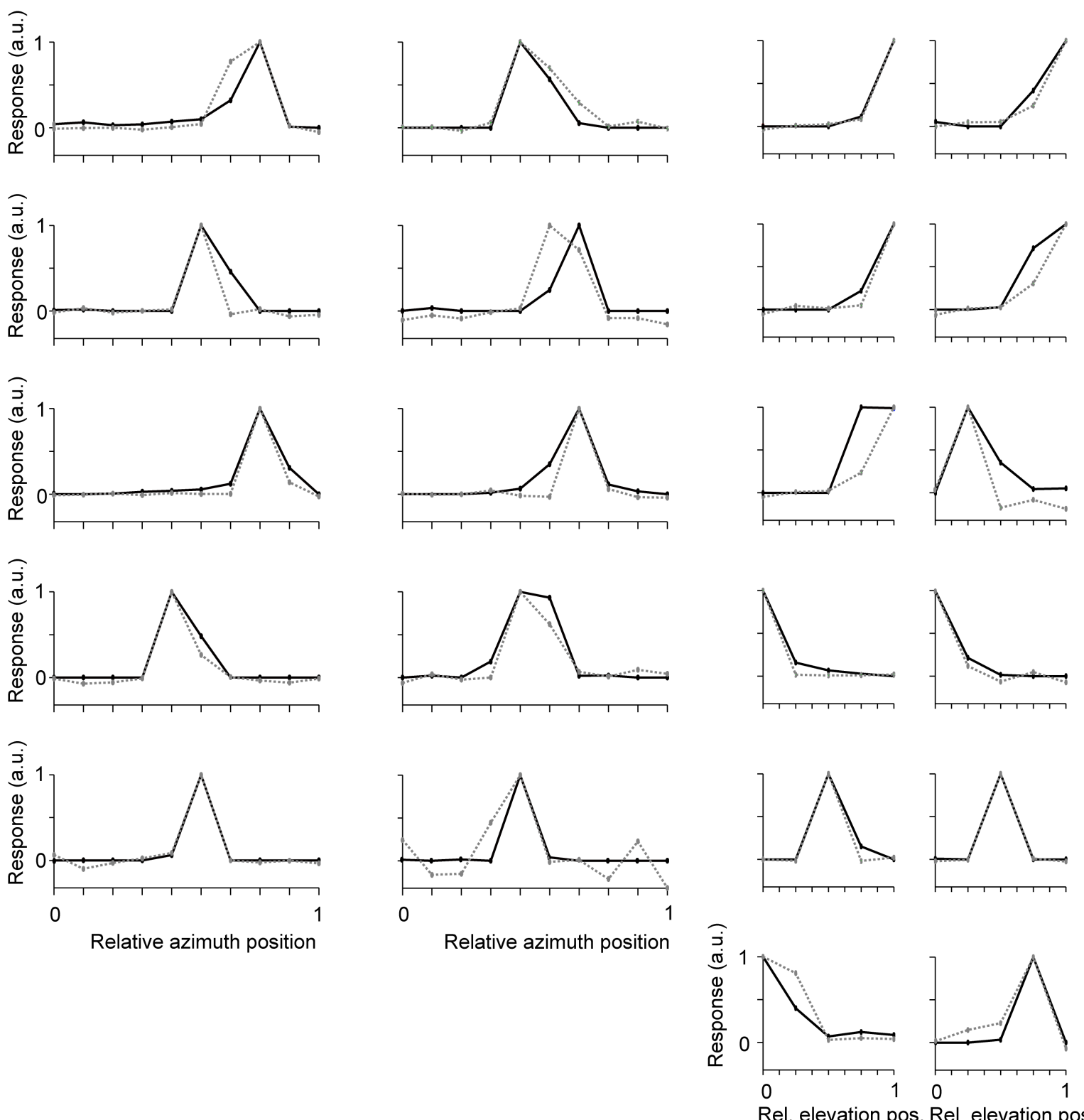

Figure 3.2 Spatial profile controls. a Compare to Fig. 3.1 g. Deconvolved, binned and averaged $\Delta F / F$ responses to small bars moving leftward across the LED display at the respective branchlet's preferred elevation, (black, solid lines), and spatial response controls using leftward-moving gratings restricted to small patches along the azimuth (grey, dotted lines). b Compare to Fig. 3.1 d. Same as in a, but for bars moving downward and patches of downward-moving gratings along the elevation.

Secondly, I asked whether local preferred directions can be reliably determined from the deconvolved responses to small bars moving in four directions. As a control, I moved a small grating in eight directions at the preferred location of a branchlet and evaluated the responses as $\Delta F / F$, allowing for the signal to decay back to baseline between the different stimulus presentations. Compared to mapping the complete receptive field of a branchlet in 
this way, the approach I chose is faster by almost an order of magnitude. Using my moving bar stimulus, the strength of the direction selectivity is considerably underestimated, as demonstrated by a significantly lower direction selectivity index (DSI, see Materials and Methods, of $0.43 \pm 0.12$ vs. $0.80 \pm 0.27$, mean $\pm s d$, for bar vs. grating stimulus; $p$-value $<0.001$, Wilcoxon's rank sum test, $n=26$ branchlets in 5 cells). This may be attributed to a flicker response component elicited by a moving bar, independent from the direction of motion. Experiments with small grating patches revealed that responses to the appearance of the stationary grating were highly variable between branchlets, but on average amounted to $20 \pm 17 \%$ (mean $\pm \mathrm{sd}$ ) of the responses to grating motion in the preferred direction (Fig. 3.1j). Importantly, though, the local preferred directions calculated with the two methods are not statistically different (Fig. 3.1k). Taken together, I can accurately and efficiently determine both spatial sensitivity and direction selectivity with the described receptive field mapping method.

\subsection{Small dendritic branchlets have local receptive fields with clear direction selectivity}

Having established an efficient method to measure receptive fields with calcium imaging, I was able to determine the receptive fields of fine branchlets across the entire dendritic trees of individual VS cells. An example experiment on a VS 4 cell is shown in Fig. 3.3. Throughout the dendritic tree, small branchlets respond locally and with clear direction selectivity. This is true for all analyzed branchlets across VS 2-9. The average DSI is $0.5 \pm 0.2$ (mean $\pm s d, n=270$ branchlets in 24 cells) at the stimulus location with the maximal response vector length, which is likely an underestimation due to the contribution of direction-independent flicker responses. Typically, dendritic branchlets respond to bar motion in 1-2 grid fields with at least a half-maximal response, which corresponds to a visual area ranging from $18 \times 18^{\circ}$ to $18 \times 36^{\circ}$. Even neighboring branchlets measured simultaneously often have non-overlapping receptive fields (for example, see Fig. 3.3a, inset, and Fig. 3.3b confirming that I can independently measure the responses of single dendritic branchlets. 


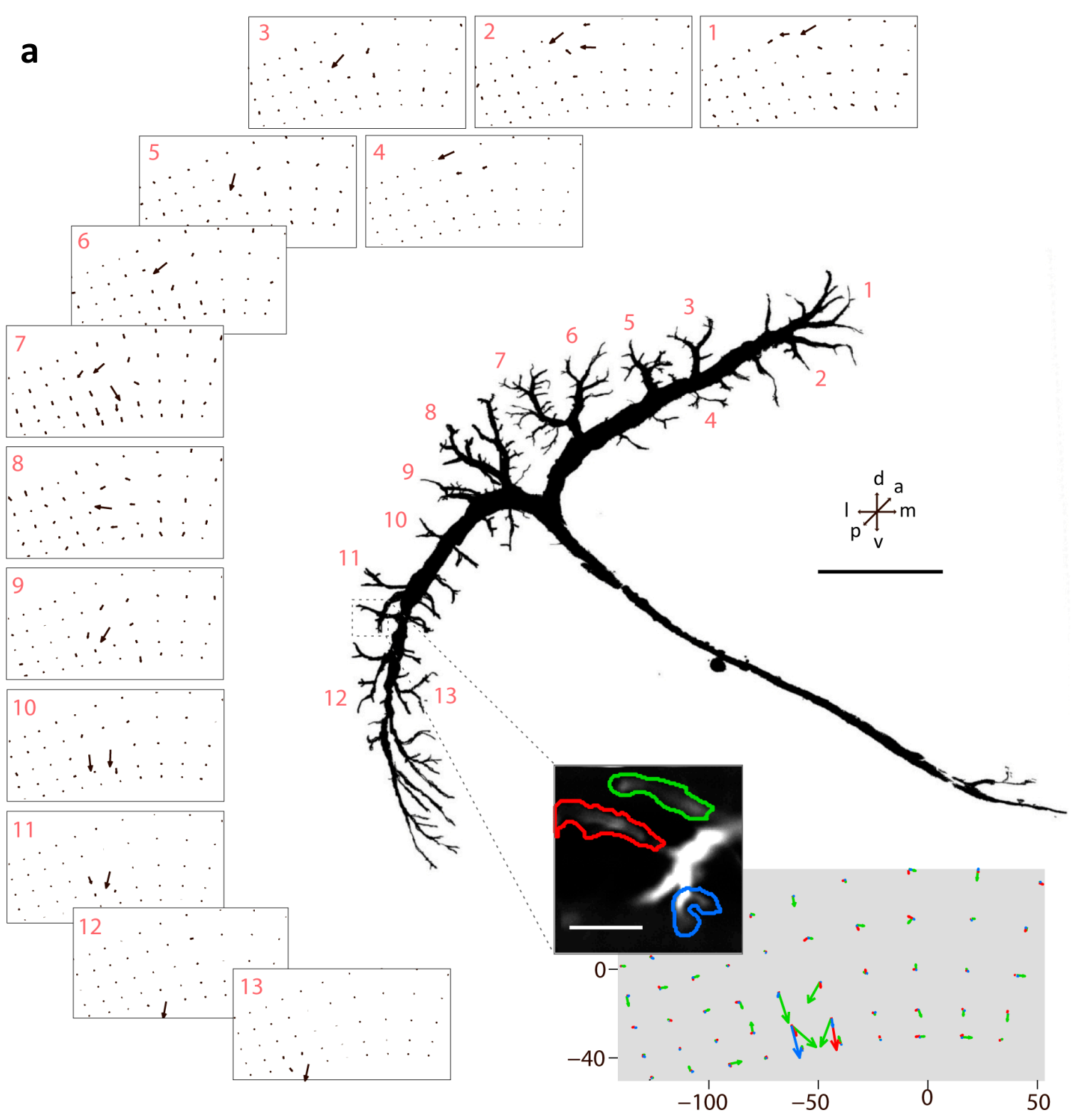

b

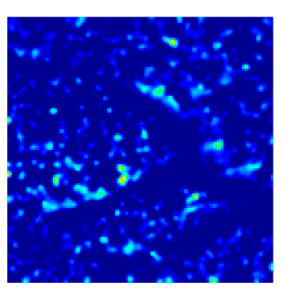

baseline

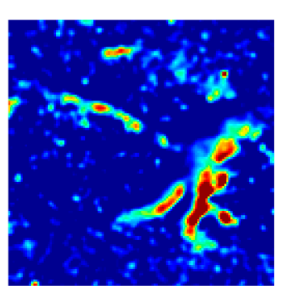

$a z=-58^{\circ}$

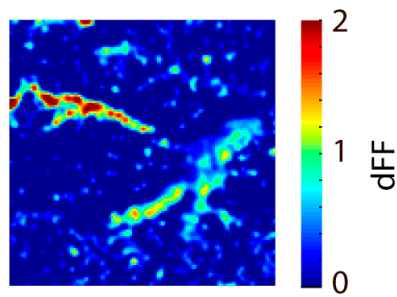

$\mathrm{az}=-41^{\circ}$

Figure 3.3 Small dendritic branchlets have local receptive fields with clear direction selectivity. a Local receptive fields of small dendritic branchlets throughout the dendritic tree of a VS 4 cell, which is depicted as a z-projection of a reconstructed 2-photon stack. Scale bar, $100 \mu \mathrm{m}$. D: dorsal, $\mathrm{v}$ : ventral, l: lateral, m: medial, p: posterior, a: anterior. The orientation of the cell matches the orientation of the cells in Fig. 1.4. For clarity, only a representative selection of measured local receptive fields (13 out of 21 ) is shown. The stimulus grids are distorted as viewed from the 
perspective of the fly (see Materials and Methods). Inset: Overlay of local receptive fields of three neighboring branchlets imaged simultaneously. The ROIs of the three branchlets are depicted in an image of the area. Scale bar, $10 \mu \mathrm{m}$. $\mathbf{b} \Delta \mathrm{F} / \mathrm{F}$ images before the stimulus started (left) and in response to a bar moving downward at $-58^{\circ}$ (middle) and $-41^{\circ}$ (right) azimuth.

This local, direction-selective dendritic activity is subcellularly organized according to two general principles. First, preferred stimulus locations are represented systematically across the dendritic tree. Dorsal branchlets respond to stimuli in the upper part of visual space, whereas ventral branchlets respond to stimuli at lower elevations (Fig. 3.3a). In fact, in all cells studied, the preferred stimulus elevation relative to the center of the cell's receptive field strongly correlates with the respective branchlet's position on the dendritic tree along the dorsoventral axis (Pearson's correlation coefficient 0.88 , different from $0, \mathrm{p}$ value $<0.001, t$-test, $\mathrm{n}=270$ branchlets in 24 cells). Similarly, there is a positive correlation between the preferred stimulus' azimuthal angle and a branchlet's location on the mediolateral axis (Pearson's correlation coefficient 0.62, different from 0 , p-value $<0.001$, $t$-test, $\mathrm{n}=270$ branchlets in 24 cells). Secondly, local preferred motion directions vary gradually across the dendrite in a pattern resembling the curved optic flow trajectory seen in these cells' output receptive fields. Compare, for example, the dendritic receptive fields of the VS 4 cell in Fig. 3.3a with this cell's output receptive field [71, compare Fig. 1.8]: Local preferred directions vary gradually from horizontal in the upper part of visual space to vertical in the lower part, representing a quarter rotation.

\subsection{Visual space is retinotopically mapped onto the lobula plate}

Given the correlation between the branchlets' preferred stimulus location and their relative position on the dendritic tree of individual VS cells, I asked whether this reflects a retinotopic map in the lobula plate. VS cells 1-10 line up in a row, and it is known that their spatial sensitivity shifts accordingly along the azimuth [70]. On the other hand, a crude mapping of a motion stimulus presented at different elevations onto the dendrite of an HS cell $[92,93]$ and a VS 1 cell $[94]$ indicated a topographic organization along the elevation. In order to tie these findings together and continuously map the stimulus space onto the lobula plate, I shifted each branchlet's relative position by the position of the corresponding 
cell type in a reference set in which all VS cells were stained and reconstructed (Fig. 3.4a, see Materials and Methods). If a retinotopic mapping exists, branchlets that are neighboring in the lobula plate should also respond to neighboring locations in visual space. Indeed, pairwise distances of dendritic branchlets across the VS cell population correlate with the pairwise distances of their respective preferred stimulus locations (Fig. 3.4b). The axes of visual space appear to be systematically represented in the lobula plate (Fig. 3.4c,d), which allows us to draw isoazimuth and isoelevation contour plots of this area (Fig. 3.4e,f), revealing a retinotopic map in which azimuth and elevation are represented along the short and the long axis of the lobula plate, respectively. Taken together, motion stimuli are retinotopically represented across the dendritic trees of individual cells as well as across the cell population. 
a

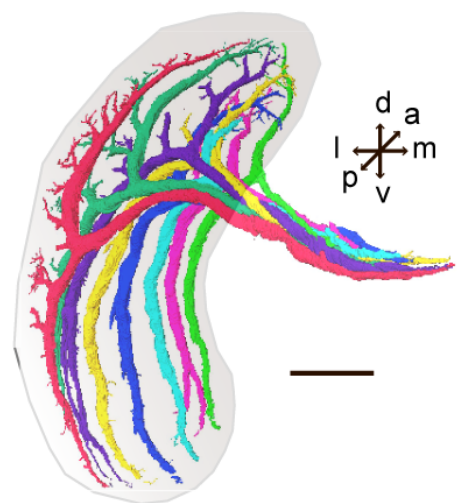

VS $2 \sim 3 m 4 \sim 5 \sim 6 \sim 7 m 8 \sim 9$ b

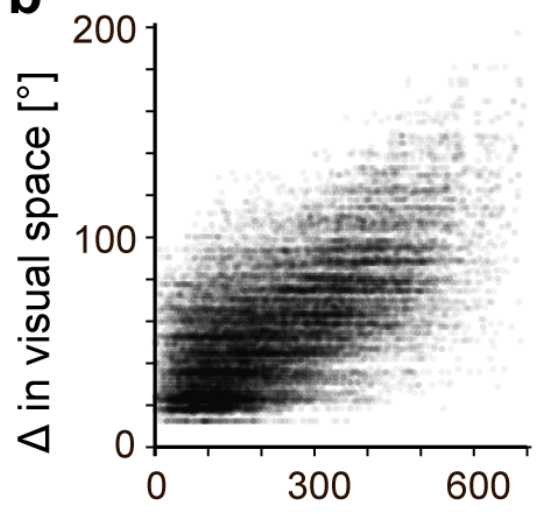

$\Delta$ in lobula plate $[\mu \mathrm{m}]$
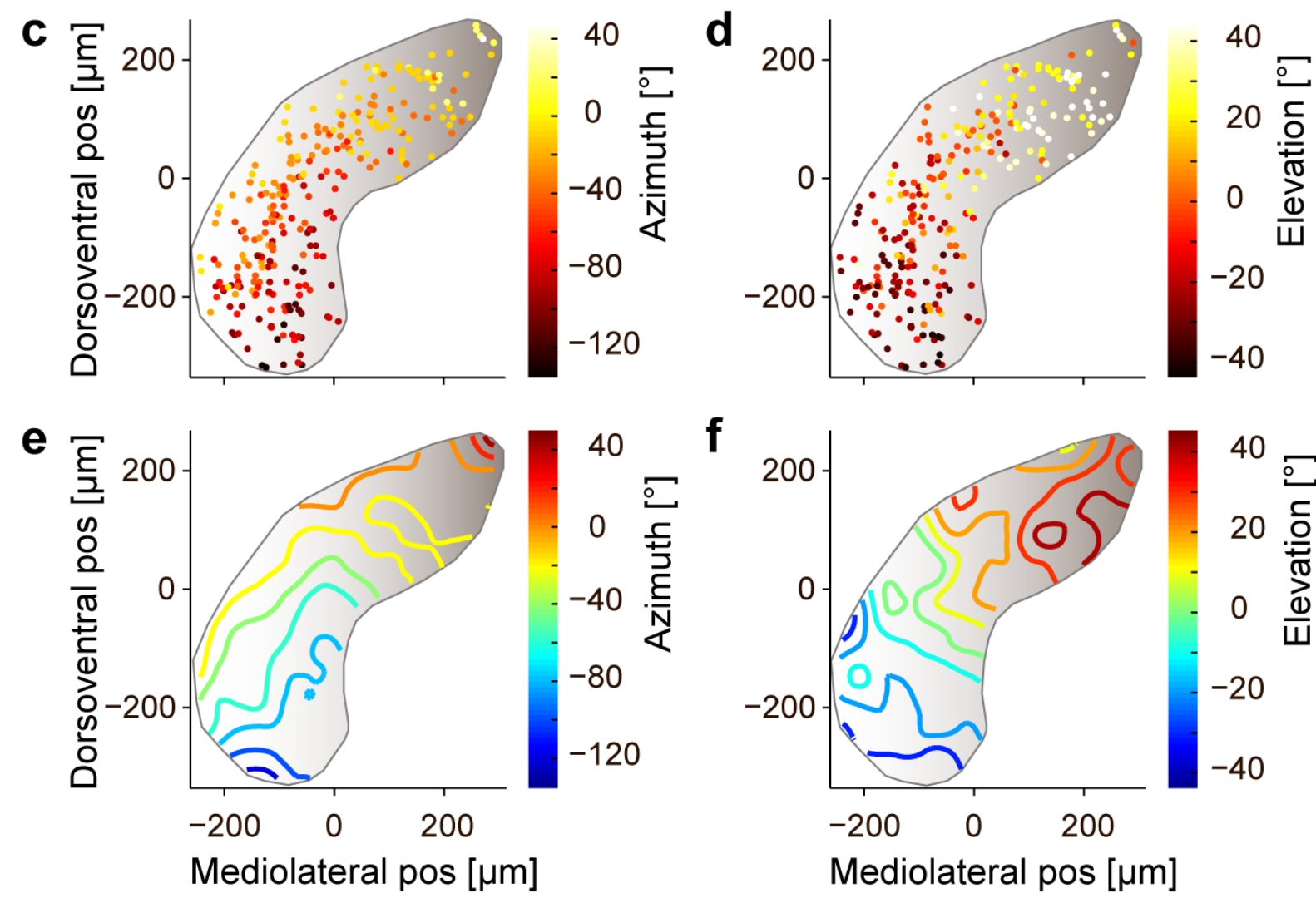

Figure 3.4 Visual space is retinotopically mapped onto the lobula plate a Reconstruction of a set of VS2-9 in the left hemisphere of a single animal. Scale bar, $100 \mu \mathrm{m}$. D: dorsal, v: ventral, I: lateral, m: medial, p: posterior, a: anterior. b Scatter plot of the Euclidian distance of two branchlets' preferred locations on a Mercator map of visual space as a function of the Euclidian distance of their centers of mass in the lobula plate. Pearson's correlation coefficient $=0.65$, different from 0 , $p$-value $<0.001$, t-test, $n=270$ branchlets, i.e. $\left(\begin{array}{c}270 \\ 2\end{array}\right)=36315$ pairs. c,d Heat maps of preferred stimulus azimuthal angle (c) and elevation location (d) as a function of the respective branchlet's relative position in the lobula plate. The orientation of the lobula plate is the same as in Fig. 1.4, Fig. 2.1 and Fig. 3.3a. e,f Contour plots of preferred stimulus azimuthal angle (e) and elevation location (f) as a function of the respective branchlet's position in the lobula plate 


\subsection{Bistratified cells exhibit a layered organization of local direction selectivity}

Next, I asked whether there is a subcellular organization of local preferred directions. Activity dependent deoxyglucose labeling of the lobula plate selectively stains four functional layers: the most anterior layer is stained in response to a grating moving horizontally from front to back, followed by layers most sensitive to back-to-front motion, upward motion and downward motion [54]. VS cells 2-6 have a planar morphology; their dendrites are confined to the most posterior layer of the lobula plate, layer 4 (compare Fig. 3.5, VS 3-5, and Fig. 3.6a). VS cells 7-9, however, are bistratified; the ventral part of their dendrite resides in layer 4 , whereas they extend their dorsal dendrite into the most anterior layer 1 of the lobula plate [compare Fig. 3.5, VS 7, and Fig. 3.6b; and compare 70,110].

In the planar VS 2-6, the distribution of local preferred directions is only mildly offset between ventral (red bars) and dorsal (blue bars) branchlets (Fig. 3.6c, p<0.001, WatsonWilliams test). Similarly, their distributions of preferred stimulus locations along the azimuth largely overlap (Fig. 3.6e). Thus, all branchlets of a VS 2-6 neuron respond to local motion in a relatively narrow part of the visual field, with a preferred direction pointing downwards and slightly front-to-back. Dorsal branchlets on average have a tendency to respond to more frontal stimuli with a slightly stronger front-to-back motion component. In contrast, ventral and dorsal dendritic branchlets in bistratified VS 7-9 exhibit opposite direction preferences as well as largely deviating distributions of their preferred stimulus location. Ventral branchlets respond to downward moving stimuli at around $-90^{\circ}$ azimuth, whereas dorsal branchlets respond to upward motion in the frontal visual field (Fig. 3.6d,f). 


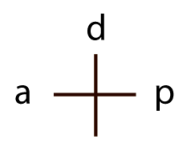

v

VS 3

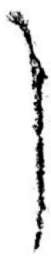

VS 4

VS 5
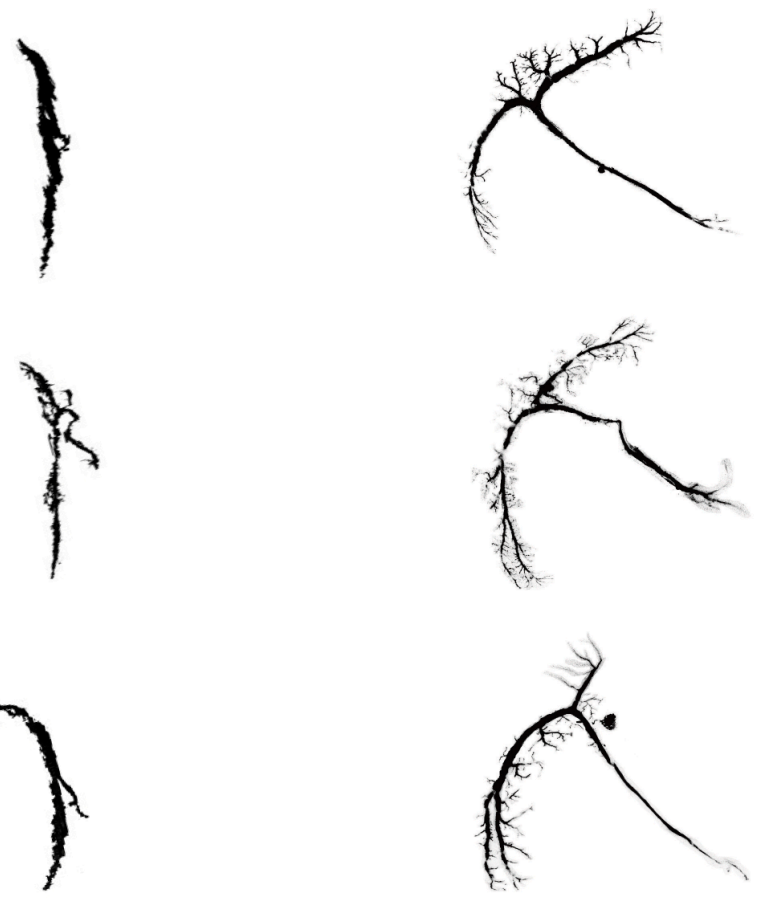

VS 7
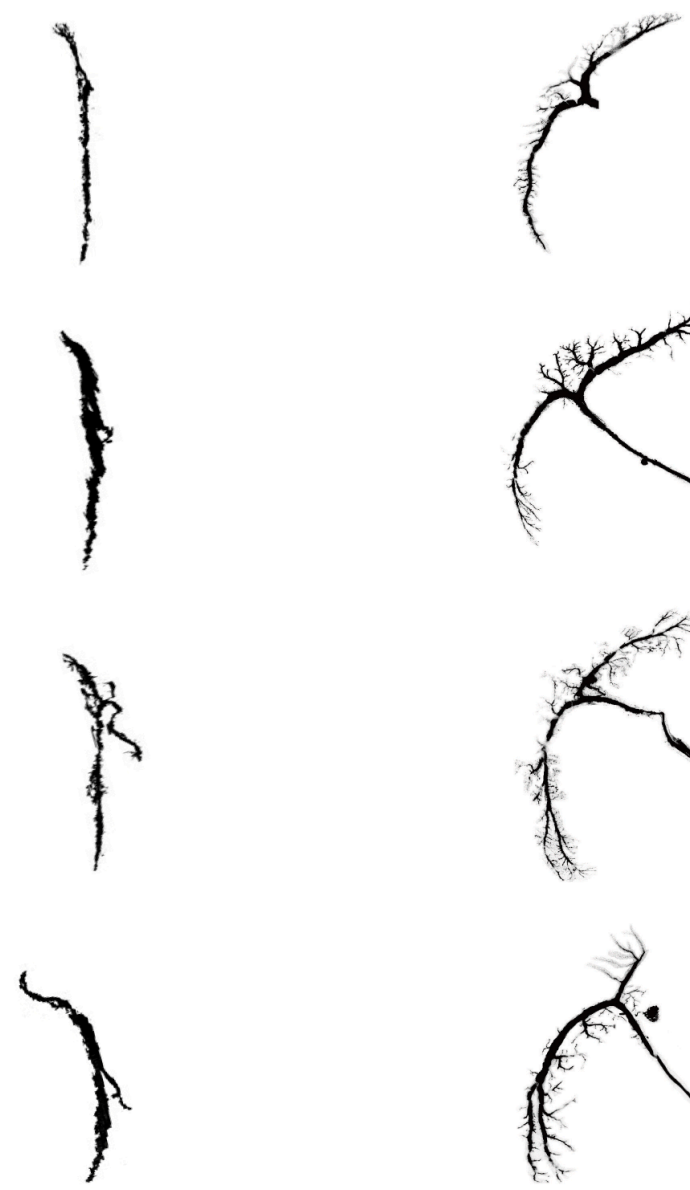

Figure 3.5 Morphology of monoand bistratified VS cells.

VS $3,4,5$, and 7 from a lateral (left column) and a posterior perspective (right column).

a, anterior, p, posterior, d, dorsal, $v$, ventral, I, lateral, $m$, medial. Note that VS 3, 4, and 5 are monostratified, whereas VS 7 extends its dorsal dendrite to a more anterior level (left column).
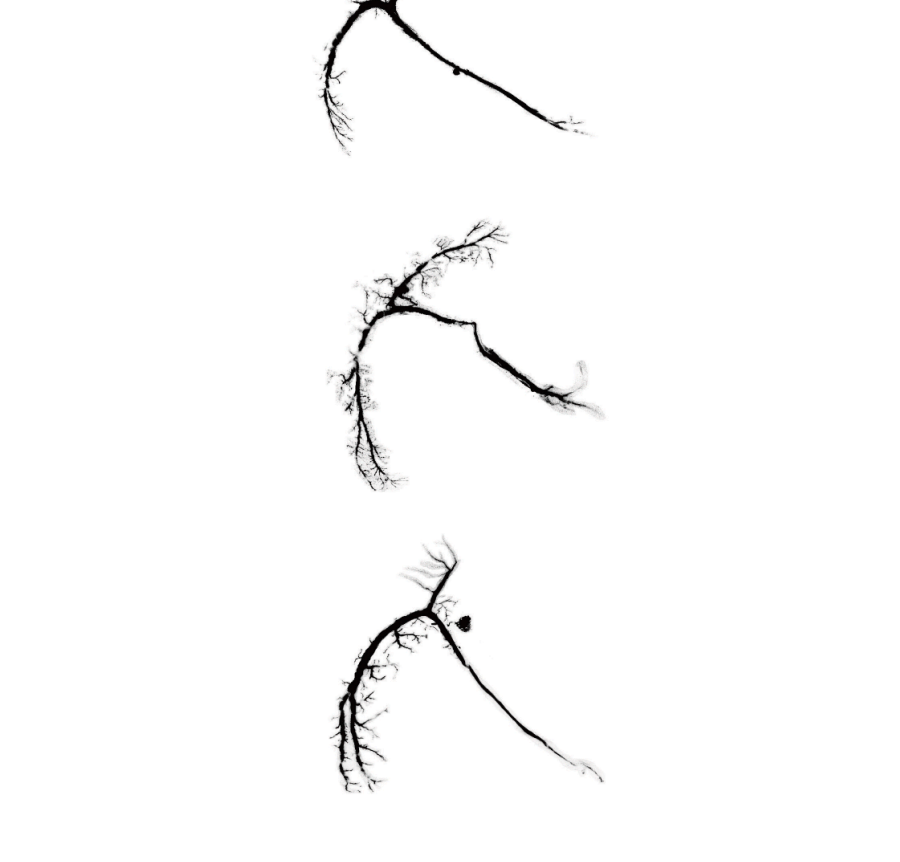
a
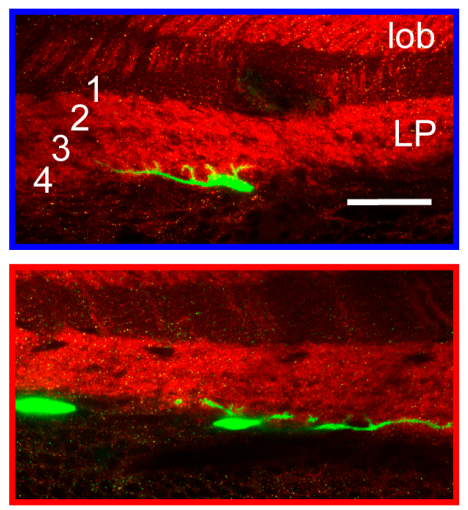

VS 2-6

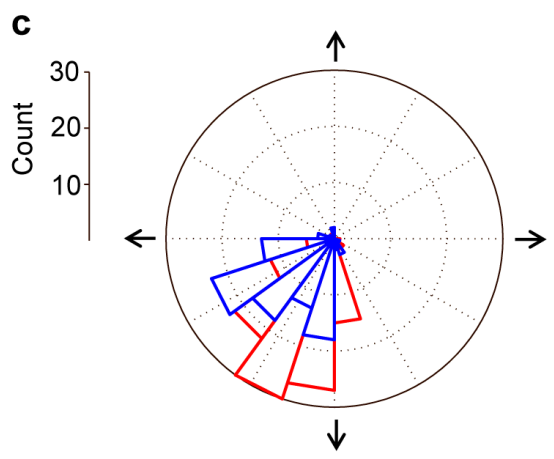

e

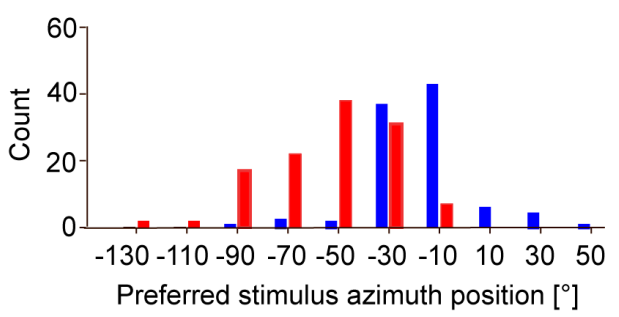

b
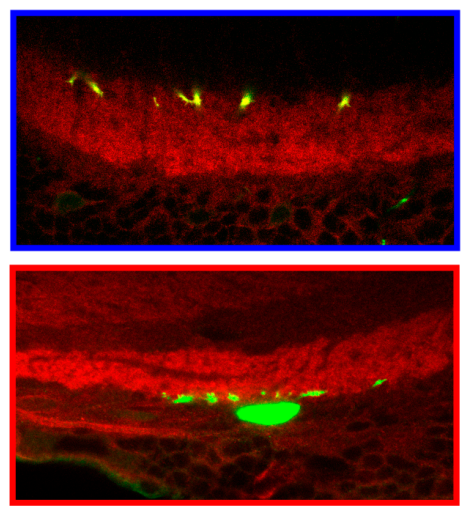

VS 7-9

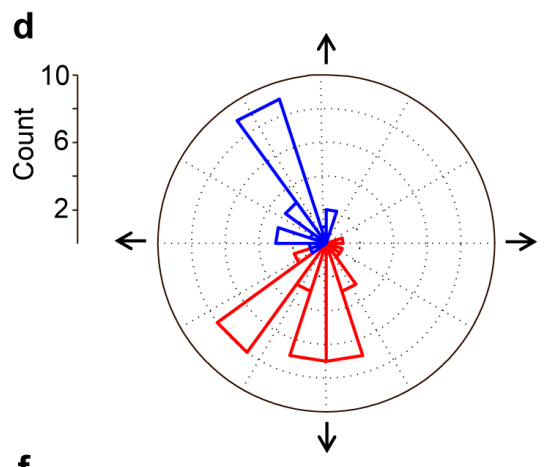

f

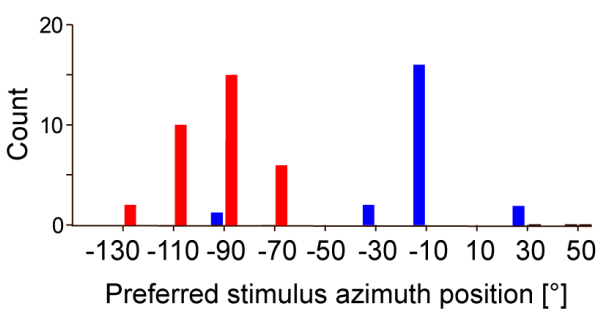

Figure 3.6 Bistratified cells exhibit a layered organization of local direction selectivity. a Immunohistochemical staining of a monostratified VS 4 cell (green), counterstained with anti-discs large for neuropil (red). Images show horizontal sections at the level of the dorsal (upper panel, blue border) and the ventral dendritic branch (lower panel, red border). LP: lobula plate, lob: lobula, numbers from 1 to 4 label lobula plate layers 1 to 4 from anterior to posterior. Scale bar, $25 \mu \mathrm{m}$. The schematic in the middle visualizes the orientation of the stained sections relative to the fly brain. LP: lobula plate, med: medulla. b Same as in a for a bistratified VS 7 neuron. c,e Distributions of local preferred directions (c) and preferred stimulus azimuthal angles (e) of branchlets located on the dorsal dendrite (blue) and the ventral dendrite (red), of monostratified VS 2-6. d,f Same as in c,e for bistratified VS 7-9 

lattice

\subsection{The intralayer distribution of preferred directions follows the deflections of the ommatidial lattice}

The most prominent feature in the spatial distribution of local preferred motion directions is the layered organization of directionally tuned inputs that I observed in bistratified cells (Fig. 3.6d). In line with these findings, it has recently been shown that four subpopulations of T4 and T5 cells are tuned to the four cardinal directions of motion, and terminate in the lobula plate in different layers depending on their preferred direction [55]: T4/T5 cells with a horizontal, front-to-back motion preference terminate in layer 1 , those with a back-to-front motion preference terminate in layer 2, layer 3 contains T4/T4 terminals with upward-, layer 4 with downward motion preference. However, I also observed a small but significant offset of the distributions of local preferred directions between ventral and dorsal branchlets in the monostratified VS 2-6 (Fig. 3.6c). Another observation that seems puzzling at first is the upward motion sensitivity of the dorsal branchlets of proximal VS cells, which are located in lobula plate layer 1 . This layer has previously been shown to be selective for front-to-back motion [54, 55]. Both these findings may be explained by the geometry of the fly's eye. The orientation of the ommatidial axes changes across the eye in a characteristic way. In particular, they are tilted by up to $90^{\circ}$ in the frontodorsal part of the visual field as compared to the lateral part [23]. If different subclasses of local motion detectors compute motion along the main ommatidial axes, their preferred directions should exhibit corresponding variations across visual space. The local preferred directions in the axonally measured receptive fields of some lobula plate cells have been shown to vary across visual space in a pattern consistent with that of the ommatidial lattice [111, 112; for illustration, see 113]. This agreement can also be seen in the dendritic activity of single VS cells. In VS 4, for example, the local preferred direction is downward in the ventral part of the receptive field, but more oblique in the dorsal part (compare Fig. 3.3a), such as the vertical rows of the ommatidial lattice in the corresponding parts of the eye.

To compare the intralayer distribution of preferred motion directions with the axes of the ommatidial lattice, I summed the receptive fields of all branchlets within lobula plate layer 4, that is, all branchlets in VS 2-6 and all ventral branchlets in VS 7-9, which reside in 
the fourth layer. The resulting global flow field is not unidirectional, but exhibits a curly structure in line with the vertical axes of the ommatidial lattice (Fig. 3.7). I conclude that local motion detectors process motion along the different ommatidial axes and project to four separate layers in the lobula plate, each of which will show a retinotopic organization and a pattern of local preferred directions that corresponds to the ommatidial lattice.

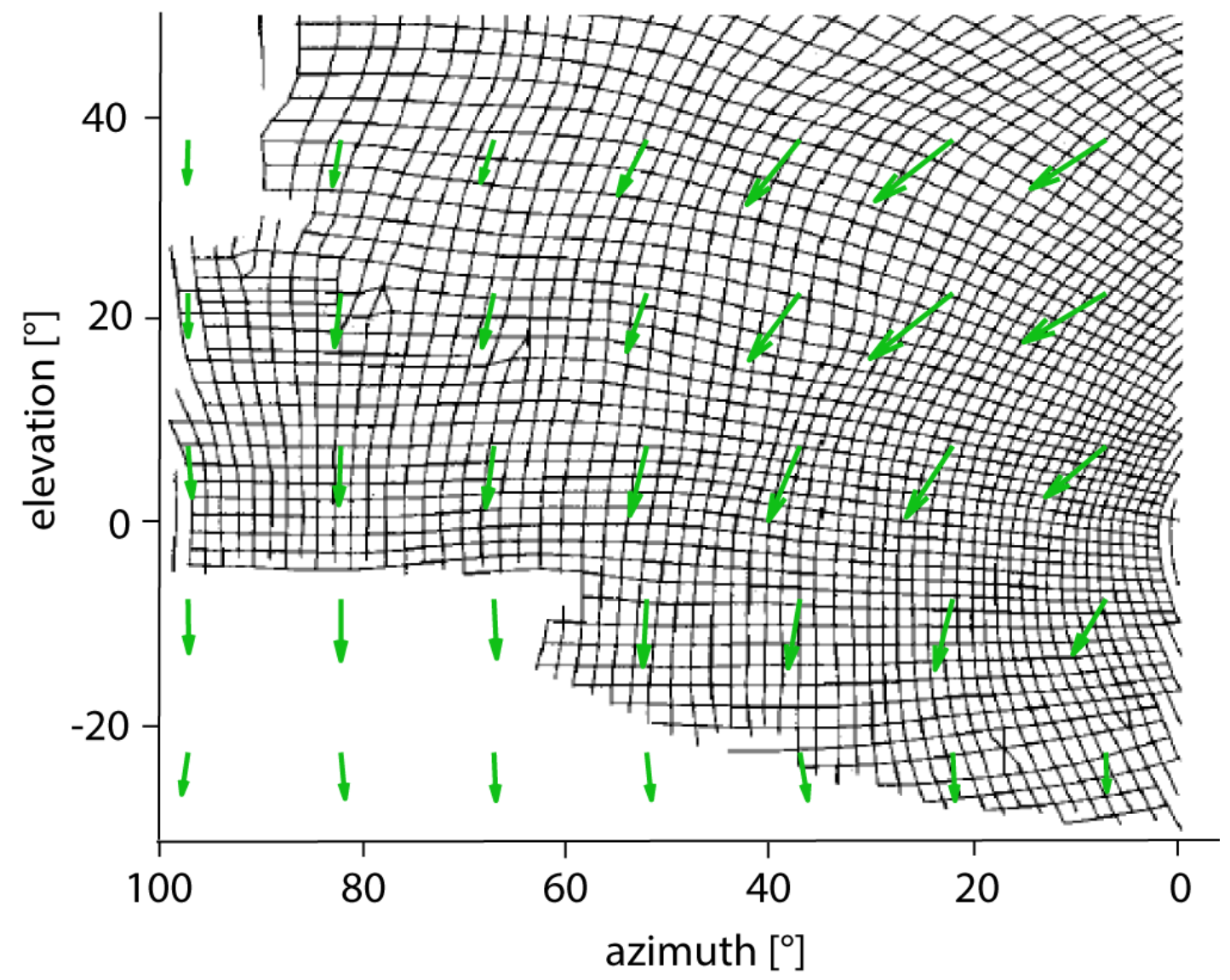

Figure 3.7 The intralayer distribution of preferred directions follows the deflections of the ommatidial lattice. Green arrows show a summation of the receptive fields of all dendritic branchlets located in layer 4 of the lobula plate, across all VS cells measured. The black grid indicates the vertical and horizontal axes of the ommatidial lattice [adapted from 23]. 


\subsection{Local dendritic receptive fields constitute the building blocks of global optic flow filters}

Given that VS cells do not only receive dendritic input, but also electrical and chemical synapses at their axons, I wanted to compare their dendritic activity to their previously published output receptive field measured in the axon [compare Fig. 2 in 71]. As a first approximation, I summed the local receptive fields over all measured branchlets of one particular cell type to obtain an average dendritic vector field for each cell type (black vector fields, Fig. 3.8). A simple sum of the dendritic receptive fields yields global optic flow patterns that reproduce the characteristic properties of the respective VS cell types' axonal output receptive fields (green vector fields, Fig. 3.8). Starting from distal towards proximal VS cells, the receptive fields become broader, they shift along the azimuth from frontal to lateral, and their rotational component becomes more pronounced. 


\section{- summed dendritic RF}

axonal RF
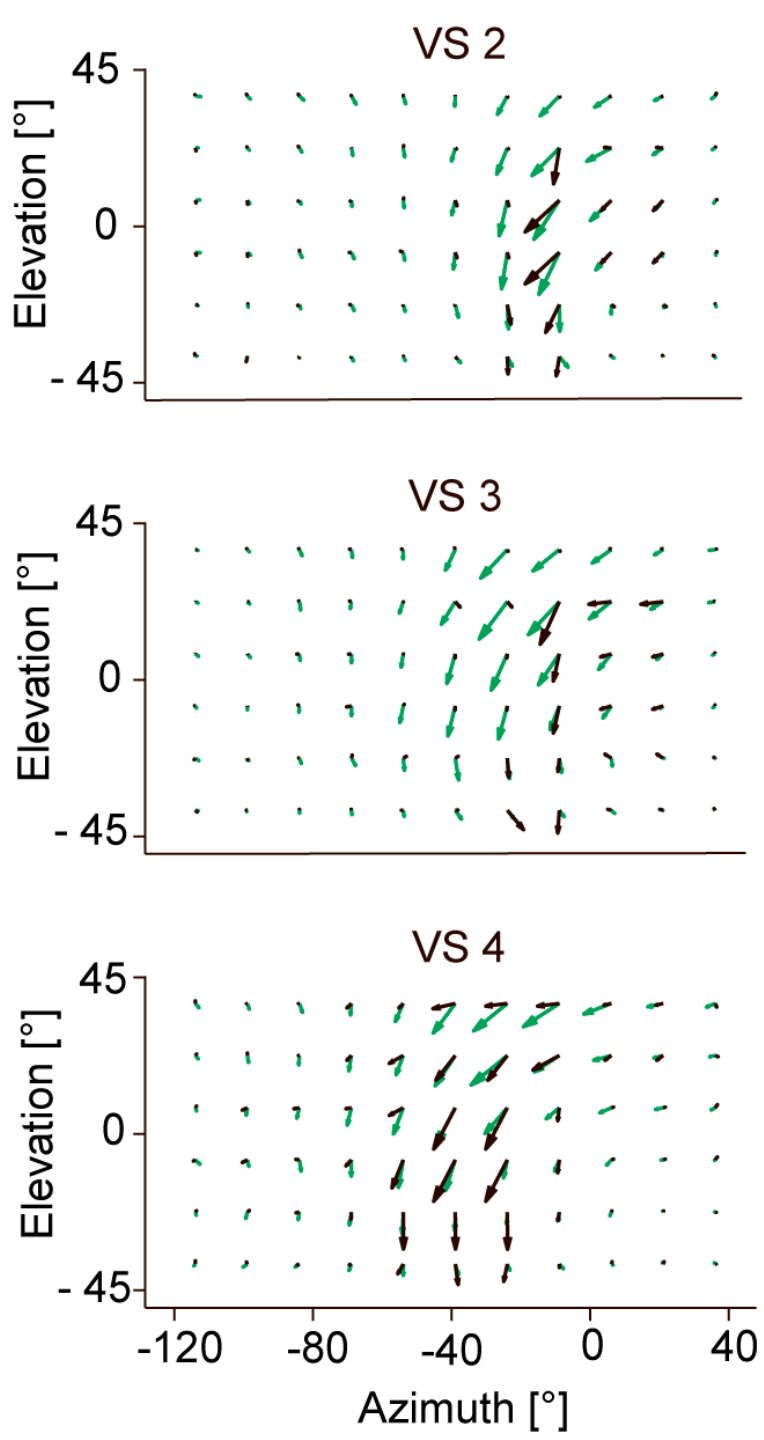
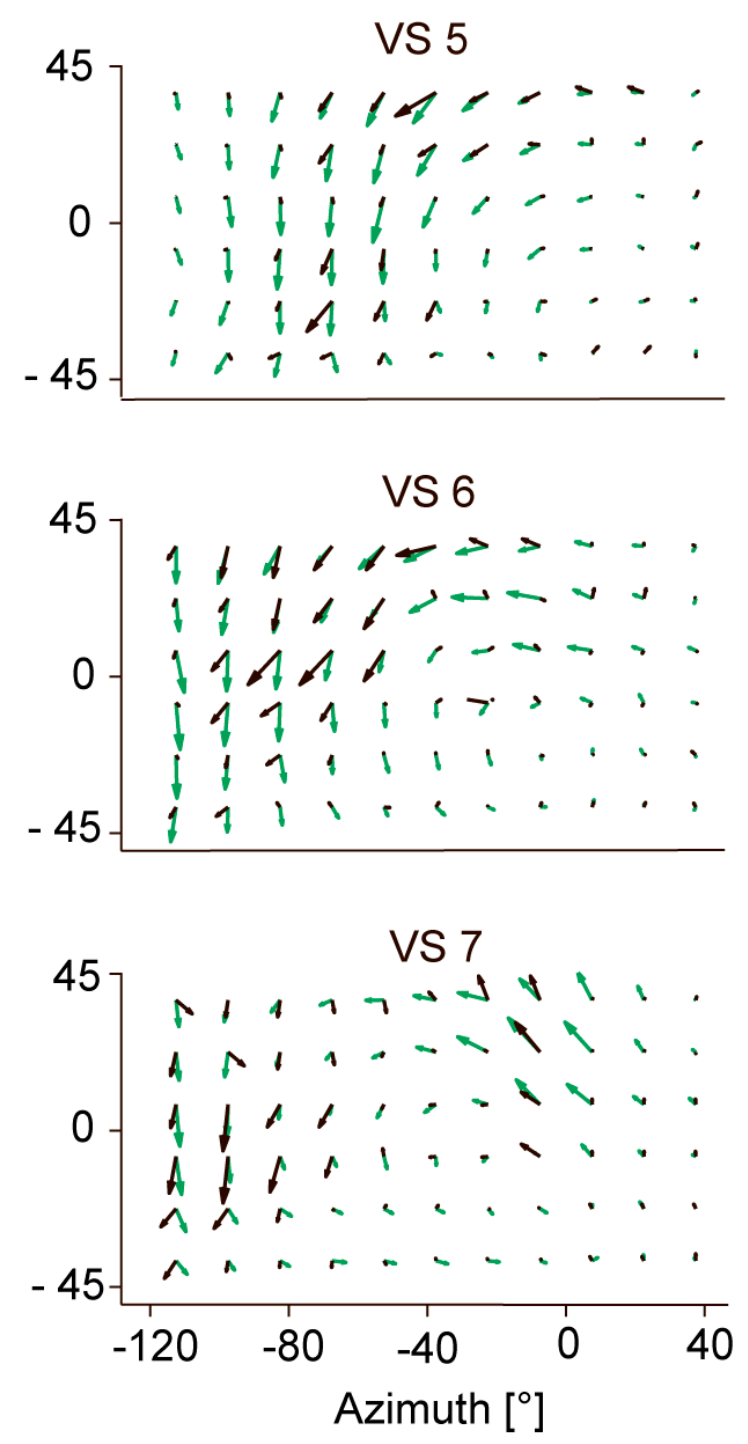

Figure 3.8 Local dendritic receptive fields constitute the building blocks for global optic flow filters.Overlay of the summed dendritic receptive fields of VS 2-7 (black), along with their output receptive fields measured electrophysiologically at the axon [data replotted with permission from 71]. The dendritic receptive fields are summed over $n$ flies and a total of $N$ dendritic branchlets for VS $2(n=1, N=10)$, VS $3(n=2, N=17)$, VS $4(n=8, N=116)$, VS $5(n=4, N=51)$, VS $6(n=2, N=22)$, VS $7(n=4, N=47)$

In a passive cable model of VS cells with a retinotopic organization, peripheral inputs arriving near the tips of their T-shaped dendrites should be attenuated compared to central inputs. However, a summation of local dendritic receptive fields regardless of their anatomical position does not overestimate local motion contributions in the periphery of the visual space compared to the center. Conceivably, active dendritic properties on VS cells 
[114] could equalize inputs at different distances to the axon as a prerequisite for the assembly of global optic flow filters that cover large parts of visual space.

As observed previously with whole field imaging [115], axonal receptive fields are generally broader than the summed dendritic ones. This results from the electrical coupling of VS cells at their axons [86] which broadens and spatially smooths their output receptive fields $[103,115]$. Compartmental modelling showed that these pooled responses should not invade the dendrites [116]. I quantitatively compared dendritic and axonal receptive fields by calculating a difference index (DI), which measures the average vector length of the difference between the two:

$$
D I(a, b)=\frac{1}{n} \sum_{i=1}^{n} \sqrt{\left(a_{x, i}-b_{x, i}\right)^{2}+\left(a_{y, i}-b_{y, i}\right)^{2}}
$$

where $\operatorname{DI}(a, b)$ is the difference index between receptive fields $a, b$. With each vector field being normalized to its maximum vector length, the $\mathrm{DI}$ can range between 0 for identical vector fields and 2 for corresponding but opposite vector fields. This measure has been introduced to compare electrophysiologically measured receptive fields of the same cell type, but determined by different authors who used different stimuli [71]. The DIs between dendritic and axonal receptive fields are of similar magnitude as those between the axonal receptive fields measured with different stimuli $[D I=0.21,0.26,0.22,0.35,0.39$ and 0.30 for VS 2-7, respectively; compare Fig. 3 in 71]. 


\section{Discussion}

Here I demonstrate that optic flow processing neurons in the fly exhibit a pronounced topographic organization of dendritic activity with respect to both spatial sensitivity as well as direction selectivity. Visual space is retinotopically mapped onto the dendritic trees of individual cells as well as onto the cell population. In addition, dendritic branchlets residing in different layers of the neuropil are tuned to different directions of motion. Within one layer, local preferred directions vary corresponding to the deflections of the ommatidial lattice. A simple sum of the local receptive fields over all dendritic branchlets within one cell type yields global receptive fields that capture the distinguishing properties of the output receptive fields measured at the axon.

\subsection{Dendritic calcium signals}

In all VS cells, I consistently observed positive fluorescence signals in response to motion of a branchlet's preferred direction, but never a consistent decrease to motion in its null direction. Previous studies disagreed on that matter. Some also found virtually no fluorescence decrease in tangential cells during ND motion [93], or specifically reported that a fluorescence decrease in response to ND motion only occurs in $\mathrm{CH}$, but not in HS or VS cells $[95,117]$. Others detected no fluorescence decreases in higher order dendrites, but observed both fluorescence increases and decreases in lower order dendrites of VS 1 cells in response to preferred and null direction motion, respectively [106, 118]. One possible explanation for this discrepancy could be that these studies relied on imaging with a CCD camera and full field illumination of the specimen, which may slightly depolarize the cells beyond their normal resting potential of $-50 \mathrm{mV}$. In a study determining the relationship between membrane potential and calcium increase, no fluorescence change could be observed in response to a voltage step from -80 to $-60 \mathrm{mV}$. Calcium started to increase for membrane potentials more positive than $-60 \mathrm{mV}$ [96]. Conceivably, depolarizing these neurons by full field illumination could shift them to a voltage range where decreases in calcium are more readily observable. 


\subsection{Deconvolution of the fluorescence traces}

In order to calculate receptive fields, I measured the impulse response in each experiment and deconvolved the calcium traces based on the inferred time constant. A mathematically precise way to do this would be Wiener deconvolution, i.e., the application of the Wiener-Kolmogorov filter to noisy measurements:

If

$$
y(t)=h(t)^{\circ} x(t)+n(t)
$$

where $y(t)$ is the measured signal, $x(t)$ is the input signal, $n(t)$ is noise which is independent from the input signal, and $h(t)$ is the unknown impulse response of the system, we want to infer $g(t)$ with

$$
x^{\prime}(t)=g(t){ }^{\circ} y(t)
$$

where $x^{\prime}(t)$ is an estimation of $x(t)$ that minimizes the quadratic error. In the frequency domain, this reads

$$
X^{\prime}(f)=G(f) \cdot Y(f)
$$

The Wiener filter offers a solution for $G(f)[119]$ :

$$
G(f)=\frac{H^{*}(f) \cdot S(f)}{|H(f)|^{2} \cdot S(f)+N(f)}
$$

where $S(f)$ is the spectral density of the signal, $N(f)$ is the spectral density of the noise, and $H^{*}(f)$ is the complex conjugate of $H(f)$. Reformulated, this reads

$$
G(f)=\frac{|H(f)|^{2}}{|H(f)|^{2}+\frac{N(f)}{S(f)}} \cdot \frac{1}{H(f)}
$$

In this notation, the effect of the Wiener filter becomes clear. Essentially, it is a series of two filters: the first part of the product is a denoising filter. In the noise-free case, this part would equal 1. With noise added, the signal is attenuated depending on the respective noise content at different frequencies. The second part is simply the inverse of the impulse response, corresponding to a deconvolution in the time domain. 
Not knowing the frequency content of either the input signal or the noise, I chose a heuristic approach for my analysis: First, I subtracted slow drift from the measured fluorescence signal where applicable, and smoothed the signal with a Gaussian filter. Second, I deconvolved it according to the inverse of a low pass filtered signal at a time constant estimated from the measured impulse response of the system. In effect this resembles the concise Wiener method: Both high-frequency noise and low-frequency drift in the signal are filtered out, followed by a simple deconvolution with the inverse of the impulse response function.

For comparison, Yaksi et al. [120] did calcium imaging experiments in the zebrafish with simultaneous electrophysiology. They then reconstructed the underlying firing rate changes by deconvolution of the fluorescence traces with an exponentially decaying kernel, which they fitted to the fluorescence signal corresponding to an action potential. They claimed that deconvolution was robust over a wide range of parameters, and should also yield acceptable results if simultaneous electrophysiological recordings are not possible. By contrast, the approach used here does not require simultaneous electrophysiological recordings at all. Still, the low pass characteristics of the fluorescence signal can be measured fast and efficiently in each experiment individually. This may be important when experimenting on cells with varying dye concentrations, as they can occur when the dye is loaded through a sharp microelectrode, but also in a genetically labeled cell population where expression levels vary from cell to cell. It is even feasible to measure low pass time constants in each cellular compartment during one experiment, if calcium channel types and densities are heterogeneous across one cell.

\subsection{VS cell dendritic morphologies are adapted to collect appropriate receptive field components from a spatially organized input repertoire}

I demonstrated that VS cells show a pronounced subcellular organization, with a retinotopic representation of visual space across their dendritic tree. By projecting single VS cells onto the neuropil, I could derive a retinotopic map of the lobula plate (Fig. 4.1). 


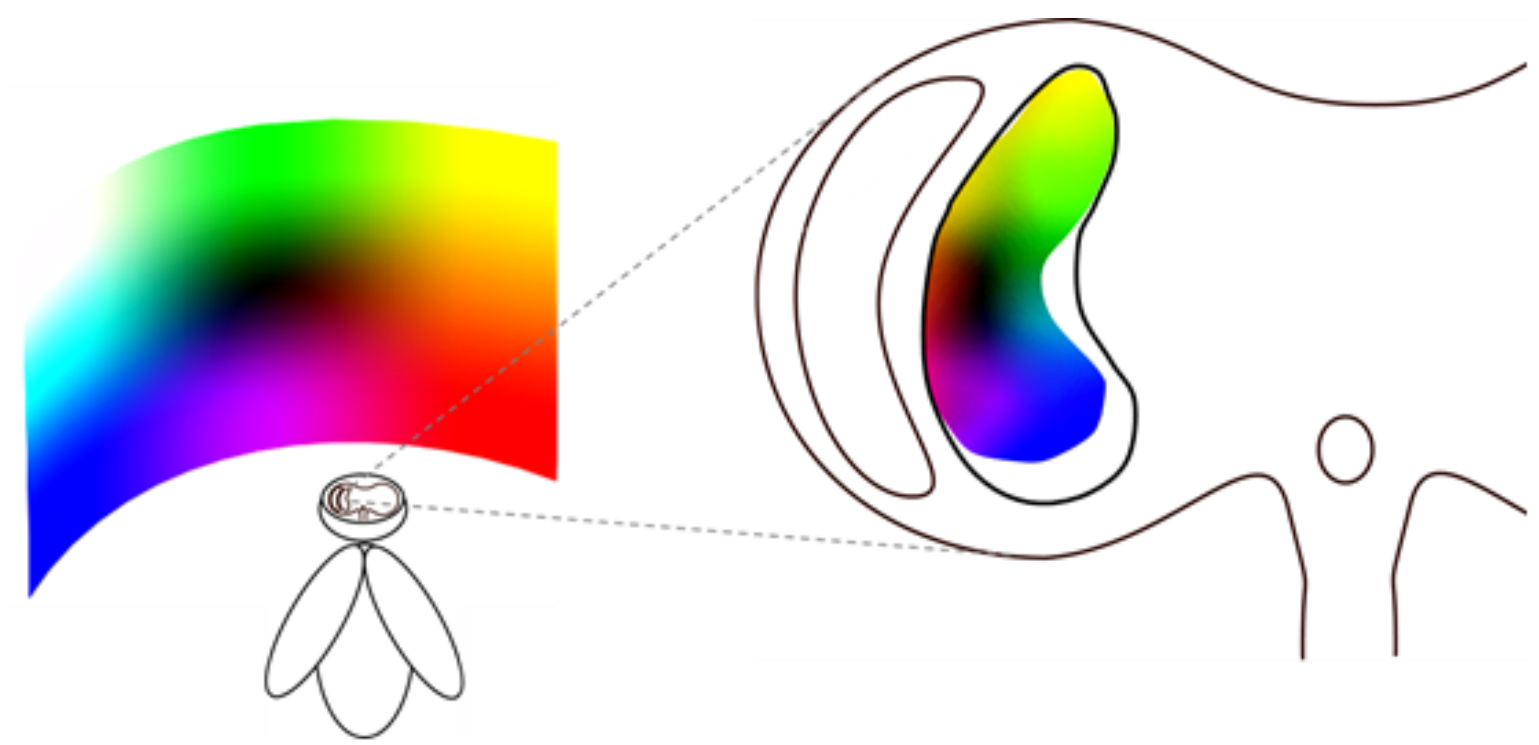

Figure 4.1 Retinotopy of the lobula plate. The color code illustrates how visual space (left) is mapped onto the lobula plate(right).

These results elaborate on previous studies that have demonstrated a retinotopic organization of VS cells on a coarser scale $[92,94]$. They also comply with the columnar layout of the bushy T-cells (T4 and T5) that provide feedforward input to VS cells $[55,56]$, and that have been suggested to project onto VS cells in an orderly manner [121]. The retinotopic relationship of the T4/T5 inputs to the lobula plate has recently been confirmed on a functional level [55].

Evidence for a subcellular mapping of visual space has also been found in other organisms. In the tectum of Xenopus larvae, a topographic bias of the dendritic response to different elevations of a flicker stimulus has been reported [8]. In the lobula giant movement detector neuron in the visual system of the locust, a retinotopic mapping has been rigorously established down to the level of single ommatidia [11].

In addition to their spatial sensitivity, I also examined the organization of local direction tuning across VS cell dendrites. The general principle of a layered direction tuning in the lobula plate is in line with previous studies that stained four different layers with cellunspecific deoxyglocose labelling in response to prolonged presentation of a grating moving in four different directions [54]. It also complies with the recent demonstration that the four different subtypes of T4/T5 cells that project to these four layers indeed show orthogonal 
direction preferences [55], and that they provide the direction selective input to LPTCs, as shown by combined silencing of T4/T5 cells and LPTC electrophysiology [55, 56]. An interesting question that remains is how and where the hexagonal ommatidial lattice is collapsed onto the orthogonal coordinate system found in the lobula plate. It has been suggested that this projection may be entailed by the L2/L4/Tm2 microcircuit [45].

I conclude that local motion tuning is computed along the axes of the ommatidial lattice, and projected onto the four layers of the lobula plate in a retinotopic manner. The VS cells' dendritic branchlets that collect these retinotopic, layered local motion inputs correspondingly show layered directional preferences with variations according to the deflection of the ommatidial axes of the compound eye (Fig. 4.2). The resulting spatial heterogeneity of direction tuning within one lobula plate layer escaped previous analyses, which have been performed with global motion stimuli and therefore averaged across locally varying preferred directions $[54,55]$. This revised model can account for both the horizontal component in the receptive fields of monostratified VS cells as well as the frontal upward motion sensitivity of bistratified VS cells. It contrasts a previous model [91] that simulated columnar feedforward input to be tuned purely to the four cardinal directions of motion, ignoring the deflection of the ommatidial rows, and ascribed intermediate, oblique preferred directions to network interactions that caused a mixing of vertical and horizontal input. 


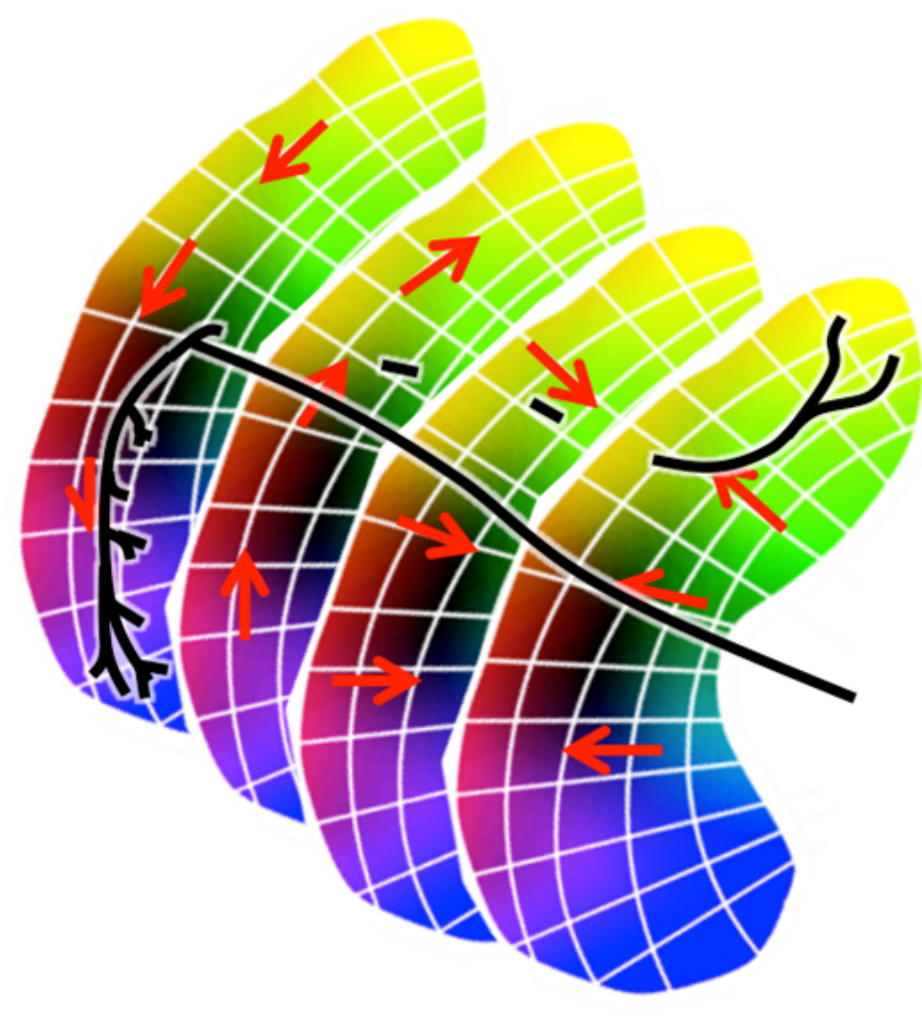

Figure 4.2 Topography of local preferred directions in the lobula plate.

The schematic illustrates the layering of local preferred directions (red arrows) in the lobula plate, with an intralayer distribution that follows the deflections of the ommatidial lattice (white lines). A bistratified VS 7 (black) extends its ventral dendrite in the most posterior, and its dorsal dendrite into the most anterior lobula plate layer.

Importantly, the rotational structure of the VS cells' output receptive fields is already present at the dendritic level. This rules out that the axonal inhibitory input from distal onto proximal VS cells is crucial for the upward motion sensitivity in their receptive fields [91]. This hypothesis was insufficient in the first place since a frontal upward motion sensitivity does not only exist computationally after the PD-ND subtraction underlying the receptive field calculation. Instead, a depolarization to frontal upward motion can be directly observed in electrophysiological recordings of these cells [compare Fig. $1 \mathrm{~b}$ in 87]. This depolarization would thus require a constant inhibition from distal VS cells, and a disinhibition following the distal VS cells' null direction, frontal upward motion, a scenario which seems unlikely. Furthermore, laser ablation of VS 1 did not abolish the response of a VS 8 cell to frontal upward motion [103]. Two potential sources are left for the excitatory, upward motion sensitive input to the dorsal dendrites of proximal VS cells within LP layer 1 : direct feedforward input from local columnar elements that terminate in this layer, and the lateral input via dendro-dendritic gap junctions with $\mathrm{dCH}$ [87], which ramifies in the same layer. In fact, dCH imports columnar input via dendro-dendritic coupling from another neuron in the lobula plate, HSN [88], which has the same preference for upward motion in the frontal part of visual space as proximal VS cells [Fig. 3d in 87]. Retinotopic columnar 
input could thus be "handed down" by HSN to $\mathrm{dCH}$ and, ultimately, VS cells. Both possibilities - feedforward columnar input and lateral, dendro-dendritic input - comply with the observation that dendritic receptive fields are locally confined and retinotopically organized across the dendritic tree, since such a subcellular organization implies that if electrical contacts are made between fine dendritic branchlets, defined subregions of receptive fields can be exchanged with preserved topography.

In summary, VS cells invade the appropriate areas of a spatially organized input neuropil to selectively pick up those local inputs that constitute the building blocks of their particular global optic flow pattern, either by making direct contacts with local columnar neurons, or by importing local inputs from other lobula plate cells via dendro-dendritic gap junctions. This way, an appropriate wiring of optic flow neurons can be achieved independent of sensory experience by simply predefining their dendritic coverage volume. In fact, the dendritic spanning field of VS cells is unaltered in flies with genetically ablated eyes [122], and flies reared in the dark show no abnormalities in the receptive field organization of lobula plate cells [123]. Similarly, in the mammalian retina, where direction selectivity occurs independent of vision [for review, see 124], an intriguing subcellular topography has been reported for directionally tuned inputs on starburst amacrine cells [7]. By contrast, in the cortex, where the establishment of direction selectivity critically depends on visual experience [for review, see 124], inputs with different directional tuning are scattered across the dendrite [4]. At the expense of plasticity, a spatial circuit organization permits the efficient hard-wired encoding of complex visual patterns, both in terms of dendritic cable length - inputs from neighboring parts of visual space can be picked up en route - as well as regarding the number of instructions that have to be genetically encoded.

\subsection{VS cells in Drosophila}

A lot of the lobula plate cell types known in Calliphora, including the vertical system cells, have also been identified in Drosophila. The lobula plate tangential cells, grouped into horizontal and vertical system (HS and VS) cells, are strikingly similar in both species. Analogous to Calliphora, Drosophila has three HS cells, HSN, HSE, and HSS (HS north, 
equatorial, and south), from dorsal to ventral. The HS cells in Drosophila have a similar anatomy and response properties as in Calliphora, with the three HS cells spanning layer 1 of the lobula plate [21] and responding to horizontally moving gratings [19]. In addition, six VS cells can be found in Drosophila [18, 124]. Very similar to VS cells in Calliphora, they are mainly located in LP layer 4, with dorsal dendrites extending into layer 1 [21, 74]. Upon closer examination, most Drosophila VS cells reveal a beautifully bistratified structure, with dendritic arborizations at distinct levels on the anterior-posterior axis [125]. Only the VS 2 cell appears to be confined exclusively to one LP layer. As for Calliphora, they mainly respond to vertical grating motion, their broad receptive fields shift along the azimuth from VS 1 to VS 6, and neighboring cells appear to be electrically coupled [18]. Their optic flow receptive fields have an evident rotational component for VS 5 and 6, whereas VS 1-4 responded virtually only to vertical motion throughout the part of visual space measured [126]. However, it is not straightforward to draw conclusions regarding the lack of a rotational component in the receptive fields of VS 1-4, since their optic flow fields have only been measured from $-50^{\circ}$ to roughly $100^{\circ}$, and VS 1 , for example, might show a horizontal motion preference beyond $100^{\circ}$, as is the case in Calliphora. Thus, at this point no comprehensive statement can be made about the connection between the anatomy of individual Drosophila VS cells, their layering and their receptive field properties. However, given the similarities in VS cell anatomy and physiology in Calliphora and Drosophila the results from this thesis most likely extend to Drosophila. 


\section{References}

1. Penfield, W. and T. Rasmussen, The cerebral cortex of man; a clinical study of localization of function. 1950, New York,: Macmillan. xv, 248 p.

2. Hubel, D.H. and T.N. Wiesel, Sequence regularity and geometry of orientation columns in the monkey striate cortex. J Comp Neurol, 1974. 158(3): p. 267-93.

3. Bosking, W.H., et al., Orientation selectivity and the arrangement of horizontal connections in tree shrew striate cortex. J Neurosci, 1997. 17(6): p. 2112-27.

4. Jia, H., et al., Dendritic organization of sensory input to cortical neurons in vivo. Nature, 2010. 464(7293): p. 1307-12.

5. Varga, Z., et al., Dendritic coding of multiple sensory inputs in single cortical neurons in vivo. Proc Natl Acad Sci U S A, 2011. 108(37): p. 15420-5.

6. Chen, X., et al., Functional mapping of single spines in cortical neurons in vivo. Nature, 2011. 475(7357): p. 501-5.

7. Euler, T., P.B. Detwiler, and W. Denk, Directionally selective calcium signals in dendrites of starburst amacrine cells. Nature, 2002. 418(6900): p. 845-52.

8. Bollmann, J.H. and F. Engert, Subcellular topography of visually driven dendritic activity in the vertebrate visual system. Neuron, 2009. 61(6): p. 895-905.

9. Peron, S.P., P.W. Jones, and F. Gabbiani, Precise subcellular input retinotopy and its computational consequences in an identified visual interneuron. Neuron, 2009. 63(6): p. 830-42.

10. Borst, A. and J. Haag, Neural networks in the cockpit of the fly. J Comp Physiol A, 2002. 188(6): p. 419-37.

11. Hassenstein, B. and W. Reichardt, Systemtheoretische Analyse der Zeit, Reihenfolgen und Vorzeichenauswertung bei der Bewegungsperzeption des Rüsselkafers Chlorophanus. Zeitschrift für Naturforschung Part B, 1956. 11(9-10): p. 513-524.

12. Reichardt, W., Autocorrelation, a principle for the evaluation of sensory information by the central nervous system, in Sensory communication, W.A. Rosenblith, Editor. 1961, MIT Press / Wiley: New York / London. p. 303-17.

13. Borst, A., Visual motion detection in Drosophila, in Encyclopedia of Computational Neurosciences, D. Jaeger and R. Jung, Editors. 2014, Springer Press, New York. 
14. Riehle, A. and N. Franceschini, Motion detection in flies: parametric control over ONOFF pathways. Exp Brain Res, 1984. 54(2): p. 390-4.

15. Eichner, H., et al., Internal structure of the fly elementary motion detector. Neuron, 2011. 70(6): p. 1155-64

16. Borst, A., Neural circuits for elementary motion detection. J Neurogenet, 2014. 28(34): p. 361-73.

17. Haag, J., W. Denk, and A. Borst, Fly motion vision is based on Reichardt detectors regardless of the signal-to-noise ratio. Proc Natl Acad Sci USA, 2004. 101(46): p. 16333-8.

18. Joesch, M., et al., Response properties of motion-sensitive visual interneurons in the lobula plate of Drosophila melanogaster. Curr Biol, 2008. 18(5): p. 368-74.

19. Schnell, B., et al., Processing of horizontal optic flow in three visual interneurons of the Drosophila brain. J Neurophysiol, 2010. 103(3): p. 1646-57.

20. Borst, A., J. Haag, and D.F. Reiff, Fly motion vision. Annu Rev Neurosci, 2010. 33: p. 49-70.

21. Fischbach, K.F. and A.P.M. Dittrich, The optic lobe of Drosophila melanogaster. A Golgi analysis of wild-type structure. Cell and Tissue Research, 1989. 258(3): p. 441-475.

22. Strausfeld, N.J., Functional neuroanatomy of the blow fly's visual system, in Photoreception and vision in invertabrates, M.A. Ali, Editor. 1984, Plenum Press: New York. p. 483-522.

23. Petrowitz, R., et al., Arrangement of optical axes and spatial resolution in the compound eye of the female blowfly Calliphora. J Comp Physiol A, 2000. 186(7-8): p. 737-46.

24. Morante, J. and C. Desplan, The color-vision circuit in the medulla of Drosophila. Curr Biol, 2008. 18(8): p. 553-65.

25. Yamaguchi, S., C. Desplan, and M. Heisenberg, Contribution of photoreceptor subtypes to spectral wavelength preference in Drosophila. Proc Natl Acad Sci USA, 2010. 107(12): p. 5634-9.

26. Heisenberg, M. and E. Buchner, Role of retinula cell types in visual behavior of Drosophila melanogaster. J Comp Physiol, 1977. 117(2): p. 127-162.

27. Morante, J., C. Desplan, and A. Celik, Generating patterned arrays of photoreceptors. Curr Opin Genet Dev, 2007. 17(4): p. 314-9. 
28. Wardill, T.J., et al., Multiple spectral inputs improve motion discrimination in the Drosophila visual system. Science, 2012. 336(6083): p. 925-31.

29. Stavenga, D.G., Insect retinal pigments: Spectral characteristics and physiological functions. Progress in Retinal and Eye Research, 1995. 15(1): p. 231-259.

30. Braitenberg, V., Patterns of projection in the visual system of the fly. I. Retina-lamina projections. Exp Brain Res, 1967. 3(3): p. 271-98.

31. Kirschfeld, K., Die Projektion Der optischen Umwelt auf das Raster der Rhabdomere im Komplexauge von Musca. Experimental Brain Research, 1967. 3(3): p. 248-\&.

32. Hardie, R.C. and P. Raghu, Visual transduction in Drosophila. Nature, 2001. 413(6852): p. 186-93.

33. Montell, C., Drosophila visual transduction. Trends Neurosci, 2012. 35(6): p. 356-63.

34. Nassel, D.R., et al., Histamine-like immunoreactivity in photoreceptors of the compound eyes and ocelli of the flies Calliphora erythrocephala and Musca domestica. Cell Tissue Res, 1988. 253(3): p. 639-46.

35. Meinertzhagen, I.A. and S.D. O'Neil, Synaptic organization of columnar elements in the lamina of the wild type in Drosophila melanogaster. J Comp Neurol, 1991. 305(2): p. 232-63.

36. Rivera-Alba, M., et al., Wiring economy and volume exclusion determine neuronal placement in the Drosophila brain. Curr Biol, 2011. 21(23): p. 2000-5.

37. Rister, J., et al., Dissection of the peripheral motion channel in the visual system of Drosophila melanogaster. Neuron, 2007. 56(1): p. 155-70.

38. Joesch, M., et al., ON and OFF pathways in Drosophila motion vision. Nature, 2010. 468(7321): p. 300-4.

39. Clark, D.A., et al., Defining the computational structure of the motion detector in Drosophila. Neuron, 2011. 70(6): p. 1165-77.

40. Joesch, M., et al., Functional specialization of parallel motion detection circuits in the fly. J Neurosci, 2013. 33(3): p. 902-5.

41. Hardie, R.C., A histamine-activated chloride channel involved in neurotransmission at a photoreceptor synapse. Nature, 1989. 339(6227): p. 704-706.

42. Jaervilehto, M. and F. Zettler, Localized intracellular potentials from pre- and postsynaptic components in the external plexiform layer of an insect retina. Zeitschrift für vergleichende Physiologie, 1971. 75(4): p. 422-40. 
43. Laughlin, S.B. and R.C. Hardie, Common strategies for light adaptation in the peripheral visual systems of fly and dragonfly. J Comp Physiol, 1978. 128(4): p. 319340.

44. Laughlin, S.B., J. Howard, and B. Blakeslee, Synaptic limitations to contrast coding in the retina of the blowfly Calliphora. Proceedings of the Royal Society Series BBiological Sciences, 1987. 231(1265): p. 437-467.

45. Takemura, S.Y., et al., Cholinergic circuits integrate neighboring visual signals in a Drosophila motion detection pathway. Curr Biol, 2011. 21(24): p. 2077-84.

46. Farrow, K., J. Haag, and A. Borst, Nonlinear, binocular interactions underlying flow field selectivity of a motion-sensitive neuron. Nat Neurosci, 2006. 9(10): p. 1312-20.

47. Silies, M., et al., Modular use of peripheral input channels tunes motion-detecting circuitry. Neuron, 2013. 79(1): p. 111-27.

48. Tuthill, J.C., et al., Contributions of the 12 neuron classes in the fly lamina to motion vision. Neuron, 2013. 79(1): p. 128-40.

49. Takemura, S.Y., Z. Lu, and I.A. Meinertzhagen, Synaptic circuits of the Drosophila optic lobe: the input terminals to the medulla. J Comp Neurol, 2008. 509(5): p. 493513.

50. Strausfeld, N.J., Atlas of an insect brain. 1976, Berlin ; New York: Springer-Verlag. xiv, p. 214.

51. Bausenwein, B., A.P. Dittrich, and K.F. Fischbach, The optic lobe of Drosophila melanogaster. II. Sorting of retinotopic pathways in the medulla. Cell Tissue Res, 1992. 267(1): p. 17-28.

52. Shinomiya, K., et al., Candidate neural substrates for off-edge motion detection in Drosophila. Curr Biol, 2014. 24(10): p. 1062-70.

53. Borst, A., In search of the holy grail of fly motion vision. Eur J Neurosci, 2014. 40(9): p. 3285-93.

54. Buchner, E., S. Buchner, and I. Bulthoff, Deoxyglucose mapping of nervous activity induced in Drosophila brain by visual movement. Wildtype. J Comp Physiol, 1984. 155(4): p. 471-483.

55. Maisak, M.S., et al., A directional tuning map of Drosophila elementary motion detectors. Nature, 2013. 500(7461): p. 212-6.

56. Schnell, B., et al., Columnar cells necessary for motion responses of wide-field visual interneurons in Drosophila. J Comp Physiol A, 2012. 198(5): p. 389-95. 
57. Bahl, A., et al., Object tracking in motion-blind flies. Nat Neurosci, 2013. 16(6): p. 730-8.

58. Takemura, S.Y., et al., A visual motion detection circuit suggested by Drosophila connectomics. Nature, 2013. 500(7461): p. 175-81.

59. Strother, J.A., A. Nern, and M.B. Reiser, Direct observation of ON and OFF pathways in the Drosophila visual system. Curr Biol, 2014. 24(9): p. 976-83.

60. Meier, M., et al., Neural circuit components of the Drosophila OFF motion vision pathway. Curr Biol, 2014. 24(4): p. 385-92.

61. Behnia, R., et al., Processing properties of ON and OFF pathways for Drosophila motion detection. Nature, 2014. 512(7515): p. 427-30.

62. Borst, A. and M. Egelhaaf, Direction selectivity of blowfly motion-sensitive neurons is computed in a two-stage process. Proc Natl Acad Sci U S A, 1990. 87(23): p. 9363-7.

63. Borst, A., M. Egelhaaf, and J. Haag, Mechanisms of dendritic integration underlying gain control in fly motion-sensitive interneurons. J Comput Neurosci, 1995. 2(1): p. 518.

64. Mauss, A.S., et al., Optogenetic and pharmacologic dissection of feedforward inhibition in Drosophila motion vision. J Neurosci, 2014. 34(6): p. 2254-63.

65. Raghu, S.V., et al., Synaptic organization of lobula plate tangential cells in Drosophila: Da7 cholinergic receptors. J Neurogenet, 2009. 23(1-2): p. 200-9.

66. Hengstenberg, R., Spike responses of 'non-spiking' visual interneurone. Nature, 1977. 270(5635): p. 338-40.

67. Krapp, H.G. and R. Hengstenberg, A fast stimulus procedure to determine local receptive field properties of motion-sensitive visual interneurons. Vision Res, 1997. 37(2): p. 225-34.

68. Hengstenberg, R., K. Hausen, and B. Hengstenberg, The number and structure of giant vertical cells (Vs) in the lobula plate of the blowfly Calliphora erythrocephala. J Comp Physiol, 1982. 149(2): p. 163-177.

69. Cuntz, H., et al., The morphological identity of insect dendrites. PLoS Comput Biol, 2008. 4(12): p. e1000251.

70. Krapp, H.G., B. Hengstenberg, and R. Hengstenberg, Dendritic structure and receptive field organization of optic flow processing interneurons in the fly. J Neurophysiol, 1998. 79(4): p. 1902-17. 
71. Wertz, A., J. Haag, and A. Borst, Local and global motion preferences in descending neurons of the fly. J Comp Physiol A, 2009. 195(12): p. 1107-20.

72. Franz, M.O. and H.G. Krapp, Wide-field, motion-sensitive neurons and matched filters for optic flow fields. Biol Cybern, 2000. 83(3): p. 185-97.

73. Karmeier, K., H.G. Krapp, and M. Egelhaaf, Population coding of self-motion: Applying Bayesian analysis to a population of visual Interneurons in the fly. J Neurophysiol, 2005. 94(3): p. 2182-2194.

74. Heisenberg, M., R. Wonneberger, and R. Wolf, Optomotor blindh31 - Drosophila mutant of lobula plate giant neurons. J Comp Physiol, 1978. 124(4): p. 287-296.

75. Geiger, G. and D.R. Nassel, Visual orientation behaviour of flies after selective laser beam ablation of interneurones. Nature, 1981. 293(5831): p. 398-9.

76. Hausen, K. and C. Wehrhahn, Microsurgical lesion of horizontal cells changes optomotors yaw response in the blowfly Calliphora erythrocephala. Proc R Soc London B, 1983. 219(1215): p. 211-216.

77. Haikala, V., et al., Optogenetic control of fly optomotor responses. J Neurosci, 2013. 33(34): p. 13927-34.

78. Borst, A. and J. Haag, Optic flow processing in the cockpit of the fly, in Invertebrate neurobiology, G. North and R.J. Greenspan, Editors. 2007, Cold Spring Harbor Laboratory Press: Cold Spring Harbor, N.Y. p. 101-122.

79. Zbikowski, R., "Fly like a Fly" (vol 42, pg 46, 2005). IEEE Spectrum, 2006. 43(1): p. 8-8.

80. Strausfeld, N.J. and U.K. Bassemir, Lobula plate and ocellar interneurons converge onto a cluster of descending neurons leading to neck and leg motor neuropil in Calliphora erythrocephala. Cell Tiss Res, 1985. 240(3): p. 617-640.

81. Haag, J., A. Wertz, and A. Borst, Integration of lobula plate output signals by DNOVS1, an identified premotor descending neuron. J Neurosci, 2007. 27(8): p. 19922000.

82. Huston, S.J. and H.G. Krapp, Visuomotor transformation in the fly gaze stabilization system. PLoS Biol, 2008. 6(7): p. e173.

83. Strausfeld, N.J. and H.S. Seyan, Convergence of visual, haltere, and prosternal inputs at neck motor neurons of Calliphora erythrocephala. Cell Tis Res, 1985. 240(3): p. 601-615.

84. Strausfeld, N.J., H.S. Seyan, and J.J. Milde, The neck motor system of the fly Calliphora erythrocephala. Muscles and motor neurons. J Comp Physiol A, 1987. 160(2): p. 205-224. 
85. Wertz, A., J. Haag, and A. Borst, Integration of binocular optic flow in cervical neck motor neurons of the fly. J Comp Physiol A, 2012. 198(9): p. 655-68.

86. Haag, J. and A. Borst, Neural mechanism underlying complex receptive field properties of motion-sensitive interneurons. Nat Neurosci, 2004. 7(6): p. 628-34.

87. Haag, J. and A. Borst, Reciprocal inhibitory connections within a neural network for rotational optic-flow processing. Front Neurosci, 2007. 1(1): p. 111-21.

88. Haag, J. and A. Borst, Dendro-dendritic interactions between motion-sensitive largefield neurons in the fly. J Neurosci, 2002. 22(8): p. 3227-33.

89. Haag, J. and A. Borst, Recurrent network interactions underlying flow-field selectivity of visual interneurons. J Neurosci, 2001. 21(15): p. 5685-92.

90. Horstmann, W., M. Egelhaaf, and A.K. Warzecha, Synaptic interactions increase optic flow specificity. Eur J Neurosci, 2000. 12(6): p. 2157-65.

91. Borst, A. and F. Weber, Neural action fields for optic flow based navigation: a simulation study of the fly lobula plate network. PLoS One, 2011. 6(1): p. e16303.

92. Borst, A. and M. Egelhaaf, In vivo imaging of Calcium accumulation in fly interneurons as elicited by visual motion stimulation. Proc Natl Acad Sci U S A, 1992. 89(9): p. 4139-43.

93. Egelhaaf, M. and A. Borst, Calcium accumulation in visual interneurons of the fly: stimulus dependence and relationship to membrane potential. J Neurophysiol, 1995. 73(6): p. 2540-52.

94. Borst, A. and S. Single, Local current spread in electrically compact neurons of the fly. Neurosci Lett, 2000. 285(2): p. 123-6.

95. Spalthoff, C., et al., Localized direction selective responses in the dendrites of visual interneurons of the fly. BMC Biol, 2010. 8: p. 36.

96. Haag, J. and A. Borst, Spatial distribution and characteristics of voltage-gated calcium signals within visual interneurons. J Neurophysiol, 2000. 83(2): p. 1039-51.

97. Denk, W., J.H. Strickler, and W.W. Webb, Two-photon laser scanning fluorescence microscopy. Science, 1990. 248(4951): p. 73-6.

98. Zipfel WR, Williams RM, Webb WW (2003) Nonlinear magic: multiphoton microscopy in the biosciences. Nat Biotechnol 21 (11):1369-1377.

99. Svoboda K, Yasuda R (2006) Principles of two-photon excitation microscopy and its applications to neuroscience. Neuron 50 (6):823-839. 
100. Salcedo, E., et al., Blue- and green-absorbing visual pigments of Drosophila: ectopic expression and physiological characterization of the R8 photoreceptor cell-specific Rh5 and Rh6 rhodopsins. J Neurosci, 1999. 19(24): p. 10716-26.

101. Reiff, D.F., et al., Visualizing retinotopic half-wave rectified input to the motion detection circuitry of Drosophila. Nat Neurosci, 2010. 13(8): p. 973-8.

102. Pologruto, T.A., B.L. Sabatini, and K. Svoboda, Scanlmage: flexible software for operating laser scanning microscopes. Biomed Eng Online, 2003. 2: p. 13.

103. Farrow, K., A. Borst, and J. Haag, Sharing receptive fields with your neighbors: tuning the vertical system cells to wide field motion. J Neurosci, 2005. 25(15): p. 3985-93.

104. D'Errico, J. Surface fitting using gridfit. 2005.

105. Oertner, T.G., T.M. Brotz, and A. Borst, Mechanisms of dendritic calcium signaling in fly neurons. J Neurophysiol, 2001. 85(1): p. 439-47.

106. Single, S. and A. Borst, Different mechanisms of calcium entry within different dendritic compartments. J Neurophysiol, 2002. 87(3): p. 1616-24.

107. Hausen, K., W. Wolburg-Buchholz, and W.A. Ribi, The synaptic organization of visual interneurons in the lobula complex of flies. A light and electron microscopical study using silver-intensified cobalt-impregnations. Cell Tissue Res, 1980. 208(3): p. 371-87.

108. Nordstrom, K., et al., Sexual dimorphism in the hoverfly motion vision pathway. Curr Biol, 2008. 18(9): p. 661-7.

109. Borst, A. and H.D. Abarbanel, Relating a calcium indicator signal to the unperturbed calcium concentration time-course. Theor Biol Med Model, 2007. 4: p. 7.

110. Hengstenberg, R., H. Buelthoff, and B. Hengstenberg, Three-dimensional reconstruction and stereoscopic display of neurons in the fly visual system, in Functional neuroanatomy, N.J. Strausfeld, Editor. 1983, Springer: Berlin, Germany. p. 183-205.

111. Hausen, K., Monocular and binocular computation of motion in the lobula plate of the fly. Verh.Dtsch.Zool.Ges., 1981. 74: p. 49-70.

112. Hausen, K., Motion sensitive interneurons in the optomotor system of the fly. The horizontal cells - receptive field organization and response characteristics. Biol Cybern, 1982. 46(1): p. 67-79.

113. Egelhaaf, M., et al., Neural encoding of behaviourally relevant visual-motion information in the fly. Trends Neurosci, 2002. 25(2): p. 96-102. 
114. Haag, J. and A. Borst, Amplification of high-frequency synaptic inputs by active dendritic membrane processes. Nature, 1996. 379(6566): p. 639-641.

115. Elyada, Y.M., J. Haag, and A. Borst, Different receptive fields in axons and dendrites underlie robust coding in motion-sensitive neurons. Nat Neurosci, 2009. 12(3): p. 327-32.

116. Cuntz, H., et al., Robust coding of flow-field parameters by axo-axonal gap junctions between fly visual interneurons. Proc Natl Acad Sci USA, 2007. 104(24): p. 10229-33.

117. Durr, V., R. Kurtz, and M. Egelhaaf, Two classes of visual motion sensitive interneurons differ in direction and velocity dependency of in vivo calcium dynamics. J Neurobiol, 2001. 46(4): p. 289-300.

118. Single, S. and A. Borst, Dendritic integration and its role in computing image velocity. Science, 1998. 281(5384): p. 1848-50.

119. Gabbiani, F., and Cox, S.J., Mathematics for Neuroscientists. 2010, London, Burlington, San Diego, Elsevier- Academic Press, p. 338-40

120. Yaksi, E. and R.W. Friedrich, Reconstruction of firing rate changes across neuronal populations by temporally deconvolved Ca2+ imaging. Nat Methods, 2006. 3(5): p. 377-83.

121. Strausfeld, N.J. and J.K. Lee, Neuronal basis for parallel visual processing in the fly. Vis Neurosci, 1991. 7(1-2): p. 13-33.

122. Scott, E.K., J.E. Reuter, and L. Luo, Dendritic development of Drosophila high order visual system neurons is independent of sensory experience. BMC Neurosci, 2003. 4: p. 14.

123. Karmeier, K., et al., Early visual experience and the receptive-field organization of optic flow processing interneurons in the fly motion pathway. Vis Neurosci, 2001. 18(1): p. 1-8.

124. Elstrott, J. and M.B. Feller, Vision and the establishment of direction-selectivity: a tale of two circuits. Curr Opin Neurobiol, 2009. 19(3): p. 293-7.

125. Scott, E.K., T. Raabe, and L. Luo, Structure of the vertical and horizontal system neurons of the lobula plate in Drosophila. J Comp Neurol, 2002. 454(4): p. 470-81.

126. Joesch, M., Lobula plate tangential cells in Drosophila melanogaster; Response properties, synaptic organization and input channels, 2009, PhD thesis, LudwigsMaximilians-Universität München 


\section{Acknowledgements}

First of all, I want to thank Axel Borst, my PhD supervisor, for help and advice, and for being an inspiring example of what a balanced, fulfilled professional life can look like. I appreciate your support for our family over the last months! This really helped juggling the transitions in our life, from finishing the Phd to work applications and starting a family at the same time.

Many thanks to Bulle, my supervisor, for introducing me to all the fly stuff, electrophysiology, and $2 p$ microscopy, and especially for his unparalleled pragmatism. Thanks to Mark Hübener for participating in my TAC meetings.

I also want to thank Renate Gleich for fly care, Amira reconstructions of the cells shown in Fig. 3.4a, and generally being "die gute Seele" of the lab, Ursula Weber for the Richardson's staining Fig. 3.4a is based on, and Johannes Plett for engineering and maintenance of the LED display. Thanks to the MPI workshop for their creative designs for my setup, and the IT guys for support.

I thank my proofreaders Matt Maisak, who read several drafts of this manuscript, and Alexander Arenz, who provided helpful comments on an earlier version of it.

Thanks to former and current guys from p5, you were good company throughout my PhD.

Matt, thanks for believing in me, for your love and support, and for making these past years such a wonderful time. And years to come! 



\section{Eidesstattliche Erklärung}

Ich versichere hiermit an Eides statt, dass die vorgelegte Dissertation von mir selbständig und ohne unerlaubte Hilfe angefertigt ist.

München, den

(Unterschrift)

\section{Erklärung}

Hiermit erkläre ich, *

$\square$ dass die Dissertation nicht ganz oder in wesentlichen Teilen einer anderen Prüfungskommission vorgelegt worden ist.

$\square$ dass ich mich anderweitig einer Doktorprüfung ohne Erfolg nicht unterzogen habe.

$\square$ dass ich mich mit Erfolg der Doktorprüfung im Hauptfach

und in den Nebenfächern

bei der Fakultät für der

(Hochschule/Universität)

unterzogen habe.

dass ich ohne Erfolg versucht habe, eine Dissertation einzureichen oder mich der Doktorprüfung zu unterziehen.

München, den 


\section{ELISABETH HOPP}

$\begin{array}{llll}\text { Address } & \text { Elisabeth Hopp } & \text { Phone } & \text { +49 } 1631733382 \\ & \text { Obere Clignetstr. 12 } & \text { Email } & \text { elisabethhopp@gmail.com }\end{array}$

EDUCATION

PhD in Neuroscience

09 / 2010 - 10 / 2015 LUDWIGS-MAXIMILIANS-UNIVERSITÄT, Munich PhD in Neuroscience (10 / 2015)

Diploma in Biology

09 / 2003 - 03 / $2010 \quad$ HUMBOLDT UNIVERSITÄT, Berlin

Graduate Program in Biology, Diploma 03 / 2010

Major: Computational Biology

Minors: Neurobiology, Molecular Biology and Genetics

Second Major

09 / 2005-07 / $2008 \quad$ CHARITÉ, Berlin

Medical Neuroscience

International Graduate Program Medical Neuroscience

Summer School

$07 / 2008$

OKINAWA INSTITUTE OF SCIENCE AND TECHNOLOGY, Onna, Okinawa, Japan

Okinawa Computational Neuroscience Course

Study abroad, USA

09 / 2006-08 / 2007 UNIVERSITY OF WASHINGTON, Seattle, USA

Graduate Program Neurobiology and Behavior

Abitur $\quad 2002$

LUISENGYMNASIUM, Munich

Abitur

PROFESSIONAL EXPERIENCE

PhD $\quad 09 / 2010-10 / 2015$

Diploma thesis and work as scientific assistant

Laboratory Rotation

Laboratory Rotation

Research Internship

Hospital Internship
$03 / 2009-06 / 2010$

$03 / 2007-10 / 2008$

09 / $2006-01 / 2007$

$08 / 2004-10 / 2004$

$01 / 2003-03 / 2003$
MAX-PLANCK-INSTITUTE OF NEUROBIOLOGY, Martinsried, Dpt. Circuits-Computation-Models

MAX-PLANCK-INSTITUT FOR BIOLOGICAL

CYBERNETICS, Tübingen, Network Imaging Group

UNIVERSITY OF WASHINGTON, Seattle, Dept. of Physiology and Biophysics

UNIVERSITY OF WASHINGTON, Seattle, Dept. of Psychiatry and Behavioral Sciences

HOSPITAL OF THE LUDWIGS-MAXIMILIANSUNIVERSITÄT, Munich, Research group „Functional magnetic resonance imaging: integration and validation“

BEZIRKSKRANKENHAUS HAAR, Munich, Neurological ward and Psychiatric admissions ward 


\section{PUBLICATIONS}

\section{Journal of Comparative}

Physiology A

Nature

The Journal of Neuroscience
2014

Elisabeth Hopp, Juergen Haag, Alexander Borst

Subcellular Mapping of Dendritic Activity in Optic Flow Processing Neurons

2013 Matthew S. Maisak*, Juergen Haag*, Georg Ammer, Etienne Serbe, Matthias Meier, Aljoscha Leonhardt, Tabea Schilling, Armin Bahl, Gerald Rubin, Aljoscha Nern, Barry Dickson, Dierk Reiff,

Elisabeth Hopp \& Alexander Borst

A directional tuning map of Drosophila elementary motion detectors.

Timothy D. Hanks, Mark E. Mazurek, Roozbeh Kiani, Elisabeth Hopp \& Michael N. Shadlen

Elapsed decision time affects the weighting of prior probability in a perceptual decision task.

\section{POSTERS AND TALKS}

Poster presentation
Invited talk

\section{STIPENDS}

2005 - 2010 Stipend of the German Academic Merit Foundation

2006- 2007 Stipend of the German Academic Exchange Service

\section{LANGUAGE SKILLS}

$\begin{array}{cl}\text { English } & \text { Bilingual proficiency } \\ \text { French } & \text { Professional proficiency } \\ \text { Italian } & \text { Elementary proficiency }\end{array}$

\section{COMPUTER SKILLS}

Programming MATLAB (advanced)

Image processing Standard programs
Adobe Illustrator (intermediate), ImageJ and Adobe Photoshop (basic) MS Office (advanced in Word, Excel, Powerpoint, Outlook) 